NISTIR 8392

\title{
Evaluating the Impact of Contactless Fingerprint Imagery Spatial Loss on Matcher Performance
}

\author{
Shahram Orandi \\ John Libert \\ Bruce Bandini \\ Kenneth Ko \\ John Grantham \\ Brian Cochran \\ Craig Watson
}

This publication is available free of charge from: https://doi.org/10.6028/NIST.IR.8392

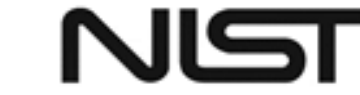

National Institute of Standards and Technology U.S. Department of Commerce 


\title{
Evaluating the Impact of Contactless Fingerprint Imagery Spatial Loss on Matcher Performance
}

\author{
Shahram Orandi \\ John Libert \\ Bruce Bandini \\ Kenneth Ko \\ Brian Cochran \\ Craig Watson \\ Information Access Division \\ Information Technology Laboratory \\ John Grantham \\ Systems Plus, Inc. \\ Rockville, $M D$
}

This publication is available free of charge from:

https://doi.org/10.6028/NIST.IR.8392

September 2021

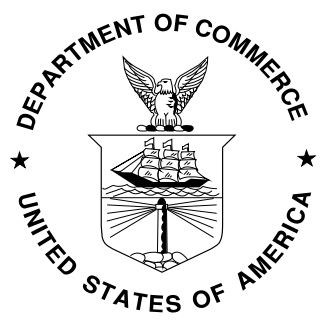

U.S. Department of Commerce Gina M. Raimondo, Secretary

National Institute of Standards and Technology James K. Olthoff, Performing the Non-Exclusive Functions and Duties of the Under Secretary of Commerce for Standards and Technology \& Director, National Institute of Standards and Technology. 
Certain commercial entities, equipment, or materials may be identified in this document in order to describe an experimental procedure or concept adequately. Such identification is not intended to imply recommendation or endorsement by the National Institute of Standards and Technology, nor is it intended to imply that the entities, materials, or equipment are necessarily the best available for the purpose.

National Institute of Standards and Technology Interagency or Internal Report 8392 Natl. Inst. Stand. Technol. Interag. Intern. Rep. 8392, 47 pages (September 2021)

This publication is available free of charge from: https://doi.org/10.6028/NIST.IR.8392 


\section{Table of Contents}

1. INTRODUCTION

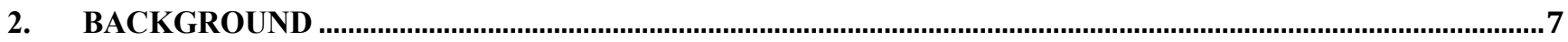

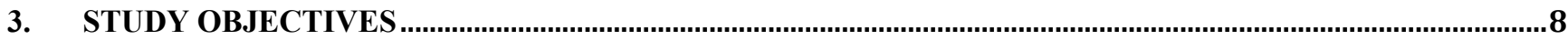

4. MATERIALS AND METHOD

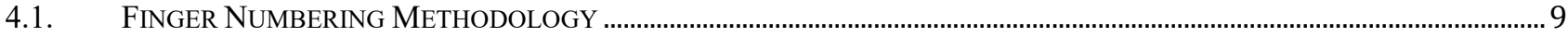

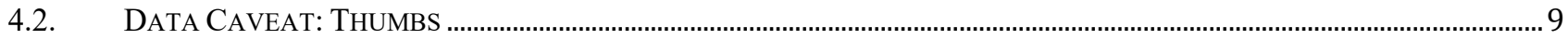

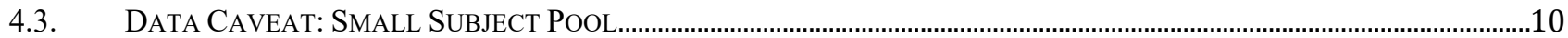

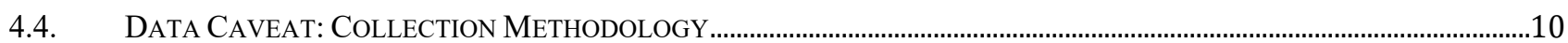

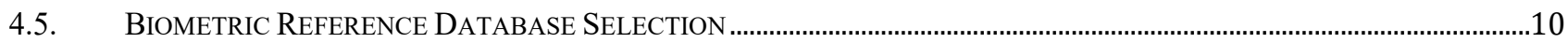

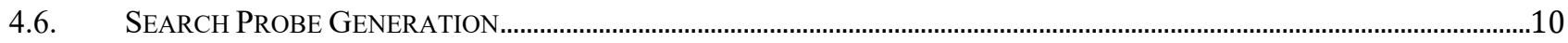

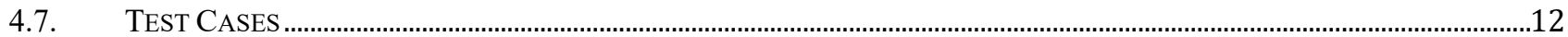

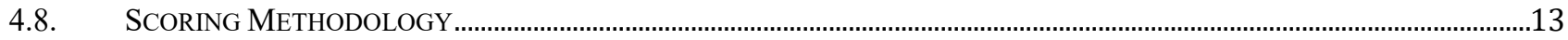

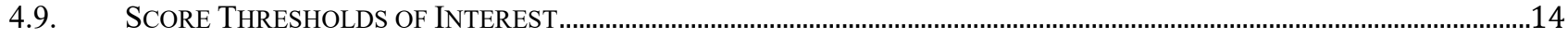

4.10. ThroughPUT CALCULATION METHODOLOGY ………………………………......................................................15

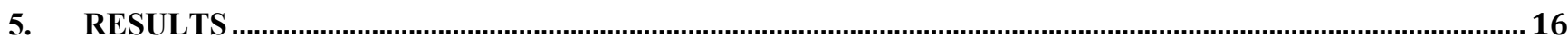

5.1. Objective 1: Baseline (Contact Database to Contact Probes) Accuracy on Ten-Print and Mobile ID

MATCHERS AT OPERATIONAL THRESHOLDS........................................................................................................................17

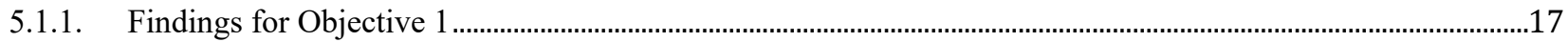

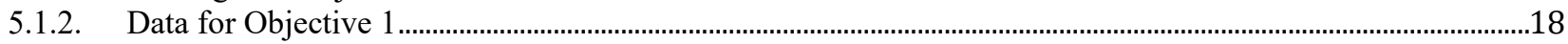

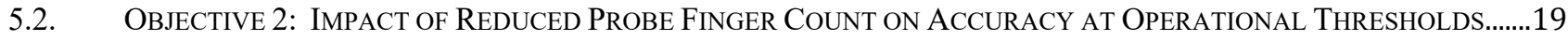

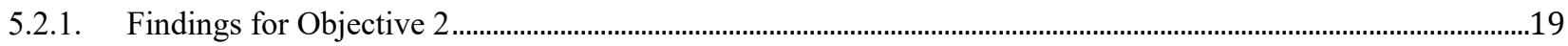

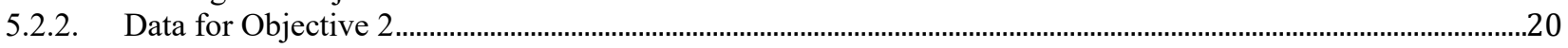

5.3. Objective 3: ImPact of Reduced Probe Finger Count on Accuracy at LeVEl Set Thresholds .............23

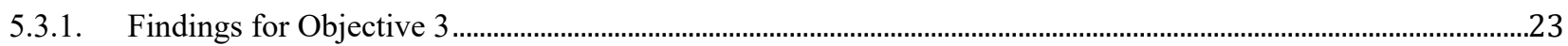

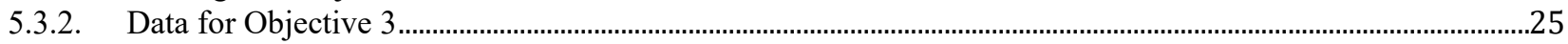

5.4. ObJeCtive 4: ImPact of Probe Image Size Reduction on Accuracy at LeVel Set ThresholdS................27

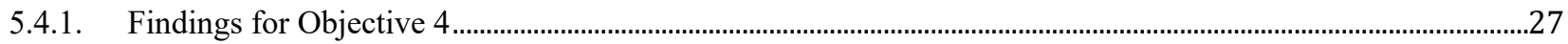

5.4.2. Data for Objective 4 ..................................................................................................................................29

5.5. OBJeCtIVE 5: COMPARISON OF MATCHER THROUGHPUT (TEN-PRINT VS. MOBILE ID MATCHER) WITH RESPECT TO

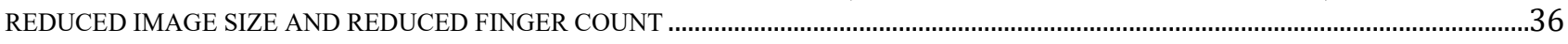

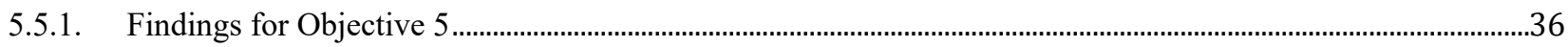

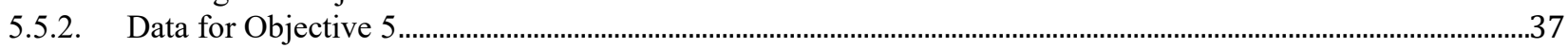

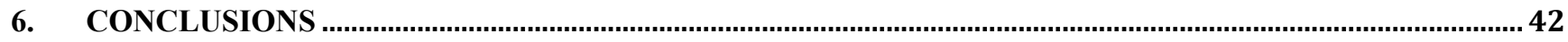

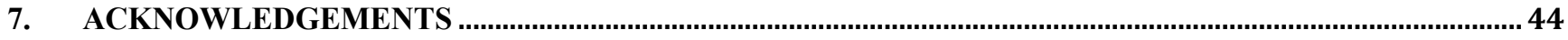

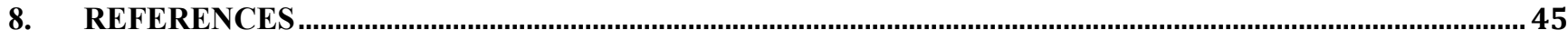




\section{List of Tables}

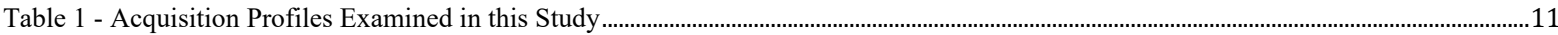

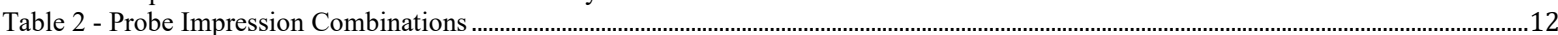

Table 3 - Summary of Test Cases Generated. …………….................................................................................................................................13

Table 4 - Scoring Threshold Values Typical in Operational Configuration ................................................................................................................14

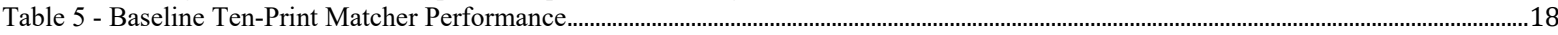

Table 6 - Baseline Mobile ID Matcher Performance..................................................................................................................................................18

Table 7 - Ten-Print Matcher Descriptive Score Data by Probe Finger Count Using a Contact-Collected Biometric Reference Database......20

Table 8 - DSCF Comparison of all Ten-Print Trials by Probe Finger Count Using a Contact-Collected Biometric Reference Database........20

Table 9 - Mobile ID Matcher Descriptive Score Data by Probe Finger Count Using a Contact-Collected Biometric Reference Database.....21

Table 10 - DSCF Comparison of all Mobile ID Trials by Probe Finger Count Using a Contact-Collected Biometric Reference Database....21

Table 11 - Ten-Print Matcher Accuracy With Respect to Reduced Probe Finger Count at Operational Thresholds. ...........................................22

Table 12 - Mobile ID Matcher Accuracy With Respect to Reduced Probe Finger Count at Operational Thresholds...........................................22

Table 13 - Ten-Print Matcher Accuracy With Respect to Reduced Probe Finger Count at Arbitrary Thresholds. .............................................25

Table 14 - Mobile ID Matcher Accuracy With Respect to Reduced Probe Finger Count at Arbitrary Thresholds...............................................25

Table 15 -Ten-Print vs. Mobile ID Matcher Performance Delta at Arbitrary Threshold With Respect to Reduced Probe Finger Count........26

Table 16 - Ten-Print Matcher Descriptive Score Data by Probe FAP Size (vs. Contact-Collected Biometric Reference Database).................29

Table 17 - DSCF Comparison of all Ten-Print Matcher Trials by Probe FAP Size (vs. Contact-Collected Biometric Reference Database)..29

Table 18 - Mobile ID Matcher Descriptive Score Data by Probe FAP Size (vs. Contact-Collected Biometric Reference Database)................30

Table 19 - Comparison of Mobile ID Matcher Trials by Probe FAP Size (vs. Contact-Collected Biometric Reference Database).................30

Table 20 - Error Differential Between Ten-Print and Mobile ID Matchers at Arbitrary Thresholds, by Size (FAP), Versus Control (FAP40).31

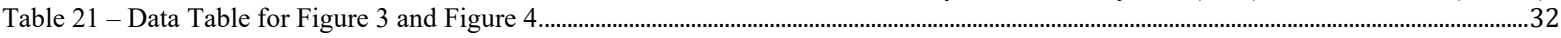

Table 22 - Count of Cases Where FNIR Improved (+) Or Degraded (-) via Image Cropping, by Probe Capture Device (vs Contact-Collected Biometric Reference Database). ...33

Table 23 - Measured Change in FNIR $(\Delta \%)$ at Level-set Threshold Resulting From Trimming Image From FAP40 Using a Contact-Collected Biometric Reference Database...

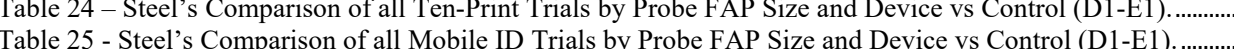

Table 26 - Steel's Multiple Comparison Rank Sum Test of Ten-Print Matcher Cases, Grouped by Probe Finger Count Versus Control Case (8F). 38

Table 27 - Steel's Multiple Comparison Rank Sum Test of Mobile ID Matcher Cases, Grouped by Probe Finger Count Versus Control Case (8F). 38

Table 28 - Steel's Multiple Comparison Rank Sum Test of Ten-Print Matcher Cases, Grouped by Probe FAP Size Versus Control Case (FAP40). 38

Table 29 - Steel's Multiple Comparison Rank Sum Test of Mobile ID Matcher Cases, Grouped by Probe FAP Size Versus Control Case (FAP40). 38

Table 30 - Ten-Print Matcher Descriptive Throughput Data by Device and Probe FAP Size Using a Contact-Collected Biometric Reference Database. ...39

Table 31 - Mobile ID Matcher Descriptive Throughput Data by Device and Probe FAP Size Using a Contact-Collected Biometric Reference Database.

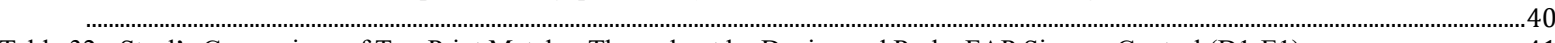

Table 32 - Steel's Comparison of Ten-Print Matcher Throughput by Device and Probe FAP Size, vs Control (D1-E1)...................................41

Table 33 - Steel's Comparison of Mobile ID Matcher Throughput by Device and Probe FAP Size, vs Control (D1-E1) .................................41

\section{List of Figures}

Figure 1 - Example of a Flat Fingerprint Impression (left) and Rolled Fingerprint Impression (right) of the Same Finger [10] .......................

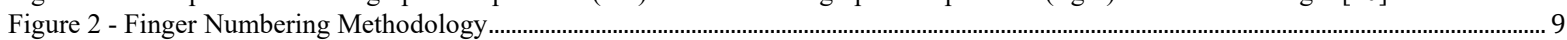

Figure 3-Q-Q plots for match scores and matcher service times on the Ten-Print and Mobile ID matcher configurations............................18

Figure 4 - Ten-Print Matcher Score Distribution by Probe Finger Count Using a Contact-Collected Biometric Reference Database..............20

Figure 5 - Mobile ID Matcher Score Distribution by Probe Finger Count Using a Contact-Collected Biometric Reference Database...........21

Figure 6 - Ten-Print Matcher Score Distribution by Probe FAP Size (vs. Contact-Collected Biometric Reference Database)............................29

Figure 7 - Mobile ID Matcher Score Distribution by Probe FAP Size. .................................................................................................................30

Figure 8 - Comparison Improved/Degraded Cases on the Ten-Print Matcher Using a Contact-Collected Biometric Reference Database Only, With Respect to Reduced Image Size by Trimming Original Image to Mobile ID FAP30, FAP20 and FAP10.

....32

Figure 9 - Comparison Improved/Degraded Cases On The Mobile ID Matcher Using a Contact-Collected Biometric Reference Database Only, With

Respect to Reduced Image Size by Trimming Original Image to Mobile ID FAP30, FAP20 And FAP10............................................32

Figure 10 - Ten-Print Matcher Score Distribution by Probe FAP Size and Device (vs. Contact-Collected Biometric Reference Database)..34

Figure 11 - Mobile ID Matcher Score Distribution by Probe FAP Size and Device Using a Contact-Collected Biometric Reference Database.

Figure 12- Matcher Throughput With Respect to the Number of Fingers, and Finger Image Size Provided as the Search Probe Using a Contact-Collected Biometric Reference Database.

Figure 13 - Ten-Print Throughput Distribution by Device and Probe FAP Size Using a Contact-Collected Biometric Reference Database.39

Figure 14 - Mobile ID Throughput Distribution by Device and Probe FAP Size Using a Contact-Collected Biometric Reference Database.40

\begin{tabular}{|c|c|c|c|c|}
\hline d:D1: & EL & ss, De & D4: & "ব": \\
\hline s Mobile Phone & D6: Contactless Mobile Phone & D7: Contactless Mobile Phone & D8: Contactless Mobile Phone & “ব”: Contactless Cases \\
\hline
\end{tabular}




\section{Executive Summary}

Observations in previous studies [7],[8] showed that contactless capture devices may be prone to capturing superfluous data (e.g., fingernail or finger background) and imaging artifacts especially around the periphery of the captured fingerprint images, as well as not being able to consistently capture certain fingers from each hand presented to them.

It was hypothesized that eliminating certain fingers that were prone to capture errors (such as the little finger of each hand) or cropping contactless fingerprint images to eliminate superfluous information such as fingernails, finger background, or distortion as a result of depth of field limitations by contactless devices may yield an improvement in the matcher's ability to make an identification as well as possibly improving the service time from the matcher.

Examining the impact of reduced finger counts showed a general trend of worsening matcher accuracy as the number of probe fingers was reduced. This trend however was not universal in that while most cases performed worse, a number of cases showed improvement. These cases that showed improvement present an opportunity for device- or matcher-specific guidance that may help improve overall system performance.

Examining the impact of reduced fingerprint image size on accuracy showed a worsening matcher performance trend as the images were progressively trimmed down. This trend was not universal, and there were notable trends for particular devices where matcher accuracy improved across all cases on that device. This again presents opportunities for operational tuning of system using device specific cases to improve matcher performance.

Finally, examination of matcher throughput shows that while there was improvement in matcher service times as the number of fingers submitted to the matcher were reduced, reduction of image dimensions (referred to as image size in the scope of this study) by trimming failed to show a clear trend, and the improvements in service times as a result of reduced image size may present gains that are too small to warrant investment in such a strategy.

The summary key findings for this study are:

- Contactless fingerprints from 200 volunteer participants were utilized on a modern fingerprint matcher designed for contact images.

- Contactless fingerprint probe images used in this study incurred penalties, in terms of both accuracy and throughput versus contact images on both the Ten-Print and Mobile ID matchers though the Mobile ID matcher fared better.

- Contactless fingerprint capture devices can be error prone when capturing certain fingers such as thumbs and little fingers. While eliminating these error prone fingers from the probe set showed improvement in some cases, an overwhelming number of cases degraded in matching accuracy.

- Contactless fingerprint capture devices may capture superfluous imagery in addition to the fingerprint. Trimming the fingerprint image yielded some accuracy benefits, but these benefits were limited to a device or specific test case. Most cases however degraded in matcher accuracy as a result of this image reduction.

- Matcher service times trended down and improved as the number of fingers in the probe set was reduced, but there was no clear trend in service time improvement with trimming of images. 


\begin{abstract}
Observations in previous studies have shown that contactless capture devices may be prone to capturing superfluous data (e.g., fingernail or finger background), distortion and imaging artifacts especially around the periphery of the captured fingerprint images, as well as not being able to consistently capture certain fingers from each hand.

This study tests the hypothesis that eliminating certain error prone fingers, or eliminating some of the periphery of all fingerprint images, may yield an improvement in the matcher's ability to make an identification as well as possibly improving the service time from the matcher.
\end{abstract}

\title{
Keywords
}

Contactless fingerprints; Touchless fingerprints; Biometrics; Accuracy; Throughput; Friction Ridge; Fingerprint Matcher Service Time

\section{Human Subjects Research}

The National Institute of Standards and Technology Institutional Review Board reviewed and approved the protocol for this project and all subjects provided informed consent. 


\section{Introduction}

In 2018, the National Institute of Standards and Technology (NIST)-in partnership and collaboration with the Federal Bureau of Investigation (FBI)-established several research tracks to examine contactless fingerprint capture, and its impact on interoperability as well as the operational impact on matching performance.

In “NIST Interagency Report (NISTIR) 8307: Interoperability Assessment 2019: Contactless-to-Contact Fingerprint Capture" [7] a thorough examination of the fundamental aspects of contactless fingerprints was conducted, as well as the ability to use these contactless images on a fingerprint matcher utilizing a biometric reference database (henceforth may be referred to simply as database) of legacy contact collected images. NISTIR 8315 [8] built on NISTIR 8307 by focusing on the operational impact of introducing contactless fingerprint imagery into a contact-collected matcher eco-system, including an independent study of contactless fingerprint imagery used both in the capacity of search images (probes) as well as background images (database), at operational thresholds as well as arbitrary thresholds.

In conducting [7] and [8], it was observed that the data captured by the contactless devices in the study did not fully meet the performance of rolled equivalent images with respect to match accuracy.

Anecdotal examination of the data used in those studies showed that some contactless devices had marked difficulty capturing all fingers presented to the device due to anthropometrics of the human hand (i.e., not capturing the little fingers since they were shorter and not fully in the field of capture), or environmental conditions (lighting, photographic background). This acquisition error also translated into segmentation and transposition errors where for example the missing finger would sometimes be replaced by the next available finger. The fingers that were impacted were predominantly the little finger, ring finger and thumb on both hands.

We hypothesized that reducing the number of contactless captured fingerprints submitted (from the full set available to 6, 4 or 2) by systematically and consistently eliminating certain fingers (such as those that were observed to be problematic from the previous studies) may improve the ability to match the subject in the database. In addition to this, it was hypothesized that reduction in finger count may also improve the matcher service time.

Furthermore, it was also observed that some of the contactless capture devices captured superfluous detail around the outer perimeter of the fingerprint pad (such as capturing the fingernail) or had difficulty capturing the friction ridge in the outer perimeter without distortion due to depth of field issues, tearing possibly due to the computational flattening of the fingerprint or other artifacts. While these contactless devices may successfully capture the entire finger, these anomalies may pose additional challenges for the matcher.

We hypothesized that cropping contactless fingerprint images slightly may eliminate the distortion in the edges of the contactless captured print thereby improving the ability to match the biometric transaction. As with reducing finger counts, it was also hypothesized that reduction of the image size (dimensions) may also improve the matcher service time.

In summary, this study examined the operational impact of utilizing contactless fingerprint imagery with reduced spatial area or reduced finger count on a fingerprint matcher's accuracy as well as throughput. 


\section{Background}

There are currently two prevalent modes of contact-based fingerprint capture employed in operations worldwide, flat fingerprint capture where friction ridge detail is captured by pressing the finger friction ridge pad down onto the capture device and then lifting, and rolled fingerprint capture where the finger is first placed down on either the left or right edge and then rolled to the other edge on the capture device capturing more of its surface area in the rolling operation (see Figure 1).

There is evidence that increased friction ridge spatial coverage such as that provided by rolled fingerprint impressions can yield better performance in certain use cases over impressions that have less spatial coverage such as plain impressions [5], [6]. The drawback to rolled fingerprint capture is that capturing each finger can take significantly longer time, can be more prone to sequence errors, and does not allow for capturing multiple fingers at the same time as do flat fingerprint captures.
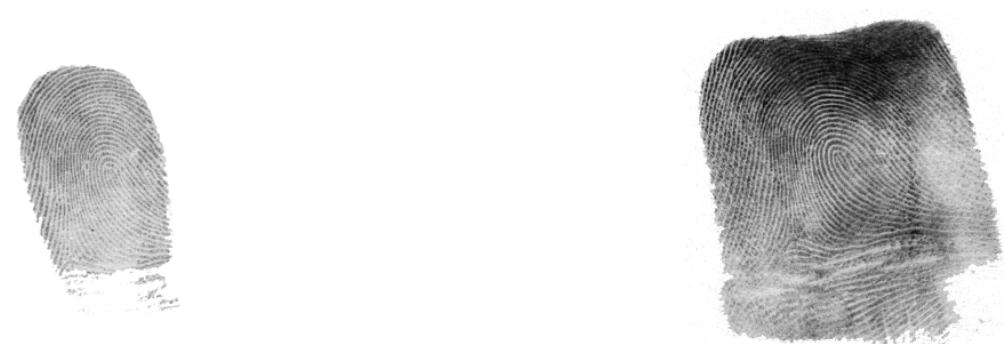

Figure 1 - Example of a Flat Fingerprint Impression (left) and Rolled Fingerprint Impression (right) of the Same Finger [10]

The goal of this study was to test the hypotheses that reducing the finger count or image spatial coverage with the contactless fingerprints may yield improvements that would otherwise not be observed with contact collected images. 


\section{Study Objectives}

The overall objective of this study was to examine the operational impact of introducing contactless fingerprint imagery on a state-of-industry high-end commercial law enforcement grade matcher that was designed to operate on contact-collected images, with a comparative analysis of matcher behavior when configured for standard Ten-Print caseloads, as well as Mobile ID caseloads.

NIST's specific objectives for this study were:

1. Establish baselines:

- Baseline matcher performance as configured in standard Ten-Print mode of operation (henceforth referred to as Ten-Print matcher) as well as Mobile ID mode of operation (henceforth referred to as Mobile ID matcher).

- Baseline matcher throughput for both configurations.

- Determine normality of distribution for both match scores and matcher service times.

2. Measure matching accuracy using a biometric reference database of mated contact-collected images on both the Ten-Print matcher as well as the Mobile ID matcher at operational thresholds utilizing contactless probe submissions with reduced finger count.

3. Measure matching accuracy using a biometric reference database of mated contact-collected images on both the Ten-Print matcher as well as the Mobile ID matcher at level-set arbitrary thresholds utilizing contactless probe submissions with reduced finger count.

4. Measure matching accuracy using a biometric reference database of mated contact-collected images on both the Ten-Print matcher as well as the Mobile ID matcher at level-set arbitrary thresholds utilizing contactless probe submissions with reduced image size.

5. Measure matcher throughput using a biometric reference database of mated contact-collected images on both the Ten-Print matcher as well as the Mobile ID matcher configuration with respect to probe submissions with reduced finger count as well as reduced image size. 


\section{Materials and Method}

The study was conducted with a block experimental design with each capture device/image type representing a treatment being examined. The mated data (image pairs that are known to be a match, and captured from the same fingers of the same participant) utilized for this investigation consisted of fingerprints provided by 200 Federal employee volunteers that were invited to participate in May of 2019.

The mated data for the 200 volunteers was comprised of approximately 1600 images (see 4.2 for caveats) and was augmented by approximately 3 million non-mated contact captured identities (or approximately 30 million images) to ensure the matcher contained an operationally realistic number of records in the biometric reference database. This biometric reference database was used for both the Ten-Print and the Mobile ID matchers.

The study utilized a state-of-industry high-end commercial law enforcement grade matcher, configured for both normal Ten-Print fingerprint identification caseloads, as well as Mobile ID caseloads. While the Ten-Print matcher is optimized to operate when a full set of rolled or flat fingerprint impressions are expected as input, the Mobile ID matcher is optimized to provide an enhanced capability to operate on fingerprints captured in the field of operation using portable or mobile capture devices. These devices may collect fewer fingerprints than a full set that is traditionally captured in a fingerprint booking environment, and/or smaller fingerprint impressions than what are traditionally collected at a booking environment due to capture platen size limitations for some portable devices. The overarching goal of this study is to examine the impact of these smaller image sizes or fewer fingerprint images being submitted from contactless devices to the matcher and the impact this may have on matcher accuracy and service time (and ultimately the throughput of the system).

\section{1. $\quad$ Finger Numbering Methodology}

The finger numbering system used in this study is that of the Henry Classification System which is the accepted norm for the industry. In the Henry Classification system fingers on the right hand are numbered from 1 to 5 starting with the thumb, and moving outward to the little finger (see Figure 2). Fingers on the left hand are numbered from 6 to 10 starting with the thumb, and moving outward.
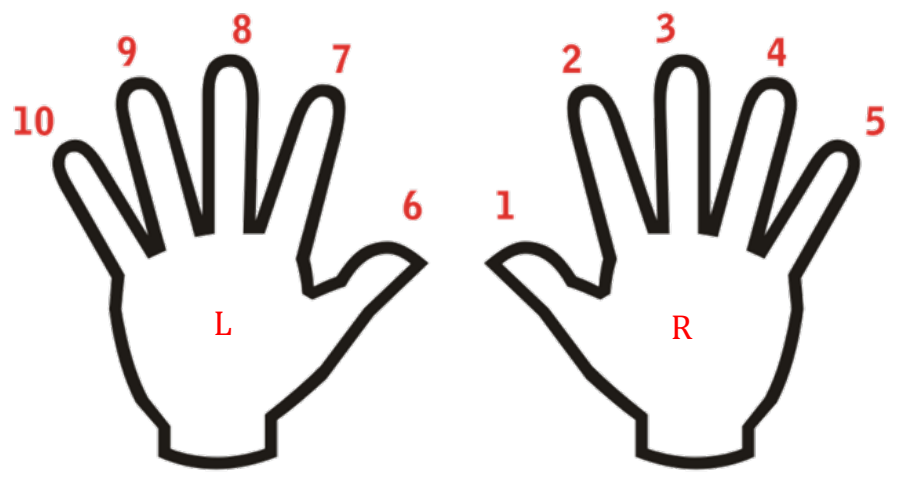

Figure 2 - Finger Numbering Methodology

\subsection{Data Caveat: Thumbs}

Contactless fingerprint capture presents unique challenges in capturing thumbs which are related to the anthropometrics of the human hand. While all contact capture devices were able to capture thumbs, some contactless capture devices were unable to capture thumb images in a timely manner given the limited resources of this study. 
In consideration of these challenges, a decision was made by the investigating team to not include images of thumbs (fingers 1 and 6 ) in the testing and analysis for both the contact and contactless capture devices.

The term "Ten-Print" in context of referring to the matcher used in this study refers to the configuration of the matcher. All data submitted to the matcher contains 8 fingers or fewer depending on the specific test case.

\subsection{Data Caveat: Small Subject Pool}

Given the limited resources both in terms of time and available volunteer subject pool, the number of subjects collected was modest at $n=200$. This subject pool further eroded by 7 due to collection challenges unrelated to the operation of the capture devices yielding $n=193$ subjects maximum.

While this small $\mathrm{n}$ allows for a meaningful examination of false negative identification rates (see 4.8) between the various capture devices and matcher configurations, false positive error measurement granularity would have been severely limited to $0.5 \%(1 / 193)$ at best and, therefore, not practical or useful in examining the performance of a modern biometric matcher. For a meaningful measurement of false positive error rates, the scale of data collection will need to be increased by at least an order of magnitude and the data partitioned so that an open-set evaluation of the matcher can be conducted.

To help maintain the fingerprint matcher at operationally realistic levels, the experimental biometric reference database was augmented by operational records (see 4.5)

\subsection{Data Caveat: Collection Methodology}

The data collection for this study was conducted in a controlled environment that facilitated the successful collection of the images. Collection of contactless images in real world settings may face challenges that were not encountered in this study.

\section{5. $\quad$ Biometric Reference Database Selection}

For each trial, the biometric reference database included up to 200 identities for a given test trial, plus 3 million additional non-mate records that are used in the NIST Fingerprint Vendor Technology Evaluation ${ }^{1}$.

During each test scenario execution, only mates from one capture device of interest (either contactless or contact based) were enrolled in the biometric reference database to avoid multiple hits for the same subject on multiple capture devices.

\subsection{Search Probe Generation}

Images from the mated-pair collection were automatically segmented, and manually verified/corrected. No rotation correction was performed on the images.

The matcher used in this study is a state-of-industry high-end commercial law enforcement grade matcher. The matcher supports both rolled fingerprints as well as flat fingerprint impression matching. The matcher also supports specific optimizations for data typical of Mobile ID.

${ }^{1}$ https://www.nist.gov/publications/fingerprint-vendor-technology-evaluation 
This matcher does not however provide any explicit support for contactless fingerprint imagery. Its operational behavior vis-à-vis contactless fingerprint imagery is provided through the generation of legacy-compatible images by contactless capture devices and is the focus of this study. This study however will not attempt to quantify how these nascent contactless capture devices generate these legacy-compatible images as the processes employed by each device manufacturer is proprietary. It is hypothesized that the process involves some level of geometric unwrapping/flattening of the curved finger surface as well as distance estimation from the device to the fingers to establish the appropriate capture resolution.

Images from the mated-pair collection were processed to produce image sets intended to simulate the various Fingerprint Acquisition Profiles or FAPs as defined in [2]. The FAPs of interest that were examined in the scope of this study are described in Table 1 below.

Table 1 - Acquisition Profiles Examined in this Study

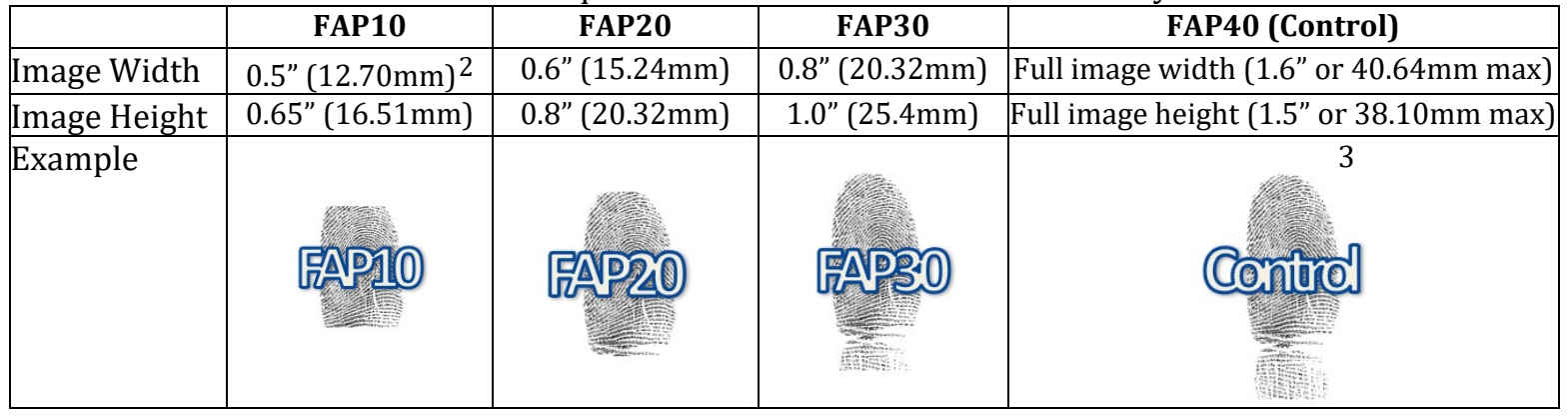

To create the simulated Mobile ID FAP images, a subsection of the finger images were cropped according to dimensions of interest for a given FAP (see Table 1). The extracted sub-section was positioned at approximately the geometric center of the finger as identified by using the calculated centroid of each digit image (after segmentation).

When the Mobile ID FAP image is a subset of the original image, the image is simply cropped from the larger image. Otherwise the (smaller) image is padded with white space (gray-scale index value of 255) to meet the size requirements of the desired FAP. The control image (also referred to as FAP40 throughput the document) is an exception where no attempt is made to pad or trim the image.

The centroid is calculated once for each Control image. This eliminates any bias from the placement of the finger for each simulated FAP10/20/30 image that is cropped and created for testing in the same way. The variance in finger placement as they are presented at capture time may have a measurable impact on matcher behavior as well, but this impact may be masked by the image cropping methodology in this study. Measuring the impact of finger placement however is outside of the scope of this study.

\footnotetext{
2 Resolution values and scanner platen dimensions are specified in inches and pixels per inch (ppi) respectively throughout this document. This is based on widely used specification guidelines for such devices and imagery, and is accepted as common nomenclature within the industry. SI units for these will be presented only once.

3 Sample images are provided to demonstrate respective FAP sizes as defined in [12]. Actual segmentation approach and friction ridge content can vary by device and capture conditions.
} 
In addition to various FAPs, probe sets were also configured for various finger impression combinations. These finger combinations are listed in Table 2.

Table 2 - Probe Impression Combinations

\begin{tabular}{|c|c|c|}
\hline Impression Case Code & Finger Numbers in Probe Set & Example \\
\hline $8 \mathrm{~F}$ & $2,3,4,5$ and $7,8,9,10$ & \\
\hline $6 \mathrm{~F}$ & $2,3,4$ and $7,8,9$ & \\
\hline $4 \mathrm{~F}$ & 2,3 and 7,8 & \\
\hline $2 \mathrm{~F}$ & 2 and 7 & \\
\hline
\end{tabular}

\subsection{Test Cases}

The study encompassed eight capture devices of interest:

D1 - An optical (Frustrated Total Internal Reflection, FTIR) contact-based Appendix-F[1] certified capture device.

D2 - An electroluminescent (EL) contact-based Appendix-F certified capture devices.

D3 - A tabletop/stationary contactless capture device.

D4 - A tabletop/stationary contactless capture device.

D5 - A mobile phone contactless capture device.

D6 - A mobile phone contactless capture device.

D7 - A mobile phone contactless capture device.

D8 - A mobile phone contactless capture device.

Note that subjects processed on D1 were captured twice, Encounter 1 (E1) and 2 (E2).

Tests were run on the matcher configured for the Mobile ID optimized mode of operation, as well as the Ten-Print optimized mode of operation.

The combinations of all test trials above that were executed on the matchers are summarized in Table 3. 
Table 3 - Summary of Test Cases Generated.

\begin{tabular}{|c|c|c|c|c|c|}
\hline \multicolumn{2}{|c|}{ Biometric Reference Database } & \multicolumn{3}{|c|}{ Probe } \\
\hline Device & FAP & Fingers & Device & FAP & Fingers \\
\hline D1-E1, & FAP40 & 8F & D1-E1, & FAP40, & $8 \mathrm{~F}$, \\
D1-E2, & & & D1-E2, & FAP30, & 6F, \\
D2 & & & D2, & FAP20, & 4F, \\
& & & D3, & FAP10 & $2 \mathrm{~F}$ \\
& & & D4, & & \\
& & D5, & & \\
& & D6, & & \\
& & D7, & & \\
& & D8 & & \\
\hline
\end{tabular}

\section{8. $\quad$ Scoring Methodology}

This study was conducted as a closed-set identification task where each subject (comprising a set of fingers, defined henceforth as the probe) being searched has an enrolled mate in the biometric reference database. The reason this study was conducted as a closed-set identification task is due to the small sample size of the contactless data that was collected (200 subjects, of which 193 were usable for the study), therefore all available subjects were used in both the biometric reference database and probe set generation.

Expected matches at Rank-1 yield the measure of hit rate [9] and candidates not returned constitute the miss rate. False Negative Identification Rate (FNIR) at candidate list Rank-1, or FNIR(1), or FNIR in the context of this study is the complement of the Rank-1 hit rate [9]. Given N enrolled subjects, with L candidates in the candidate list returned by the matcher using a threshold of $\mathrm{T}$ and rank of $\mathrm{R}$ (in this paper, $\mathrm{R}$ is always equal to 1 , and $\mathrm{L}$ is always equal to 220 per the configuration of the matcher in both Ten-Print and Mobile ID optimized modes of operation), FNIR is defined as:

$$
\operatorname{FNIR}(N, R, T, L)=\frac{\begin{array}{c}
\text { Number of mate candidate searches where } \\
\text { candidate's mate is below threshold } T \text { or below rank } R
\end{array}}{\text { Number of mate candidate lists, } S_{M}}
$$

As mentioned in section 4.4, this study utilized a very limited number of candidates in its test pool $(\mathrm{n}=193)$. While this small $\mathrm{n}$ allows for observation of FNIR behavior, the modest data available for this study did not allow for observation of false positive errors. 


\subsection{Score Thresholds of Interest}

The biometric matcher used for this study is designed to support multiple decisional thresholds. These thresholds are designed to classify results of searches into categories that facilitate a "lights-out" search (i.e., success or failure without further human intervention), as well as biometric candidates that warrant further adjudication by additional processing steps including a human examiner review (not a "lights-out" match). Furthermore, the system supports separate threshold settings for the Mobile ID and Ten-Print matcher.

For the purpose of this study, matching performance was measured according to the performance thresholds listed below in Table 4.

Table 4 - Scoring Threshold Values Typical in Operational Configuration

\begin{tabular}{|c|l|l|l|}
\hline Mobile ID & Ten-Print & Threshold Name & Notes \\
\hline $\mathbf{3 0 0 0}$ & $\mathbf{8 5 0 0}$ & Low Confidence Hit & $\begin{array}{l}\text { A candidate with a score below the low } \\
\text { confidence threshold is considered a non- } \\
\text { match, also referred to as a "Green" non-hit } \\
\text { (O). }\end{array}$ \\
\hline $\mathbf{4 1 0 0}$ & $\mathbf{1 5 0 0 0}$ & High Confidence Hit & $\begin{array}{l}\text { A candidate with a score at or above the low } \\
\text { confidence threshold, but below the high } \\
\text { confidence threshold, is considered a } \\
\text { possible match, also referred to as a } \\
\text { "Yellow" hit ( ). Candidates with a Yellow } \\
\text { hit will require additional adjudication steps } \\
\text { and manual review. }\end{array}$ \\
\hline $\begin{array}{l}\text { A candidate with a score at or above a high } \\
\text { confidence threshold is considered a "lights- } \\
\text { out" biometric candidate with no further } \\
\text { adjudication or review necessary as part of } \\
\text { the initial biometric search process. This is } \\
\text { also referred to as a "Red" hit (O). }\end{array}$ \\
\hline
\end{tabular}

In addition to the operational thresholds listed in Table 4, there are certain cases associated with test objectives where the Mobile ID and Ten-Print matchers needed to be compared at a level-set threshold to measure other aspects of matcher behavior. For this, the thresholds of $\mathbf{5 0 0 0}$ and $\mathbf{1 2 0 0 0}$ were selected completely arbitrarily with no analysis made of their fairness or fitness of use. These values were selected for cases where a level set comparison of the two matcher configurations was needed at the same exact threshold value. 


\subsection{Throughput Calculation Methodology}

When test probes were submitted into the matcher, the amount of time that each took to yield a response was recorded in a log file. Matcher throughput was calculated as the average service time for each probe submitted to the matcher.

The following caveats and conditions apply to the throughput portion of this study:

- Matcher timing data was returned at a reported resolution of 1 millisecond ( $\mathrm{ms}$ ) and is inclusive of all processing overhead for characterization of each probe on submission.

- Matcher timing data analysis does not include the characterization (i.e., vectorization) phase of the biometric reference database, enrollment, and database loading time.

- Timing data trials commenced after the matcher reached an operationally responsive state with the database loaded.

- No attempt was made to measure or remove underlying I/O overhead resulting from normal matcher operation.

- The computational environment used for this study was isolated physically and logically from the general computing environment at NIST.

- Non-essential processes were not executed on the machines involved in this study while the throughput test was being conducted.

- Matcher initialization and initial start-up times were not factored in measuring matcher performance. The test commenced with the matcher fully initialized and at idle. 


\section{Results}

The matcher's biometric reference database was initialized according to each test case being examined as described in section 4.7 , and probe sets for that given test case were submitted to the matcher for searching.

The resulting candidate lists for each test case were captured and scored according to the methodology described in section 4.8 yielding FNIR measurements for thresholds of interest (see Table 4).

The data presented in this section for each objective has been provided in a way to allow for quick visual comparison of matcher behavior under various scenarios. For additional finer-grained information, [numerical] performance data is also provided for selected scenarios.

The visualizations presented are optimized to provide large volumes of information in a relatively small space. Test cases are summarized in the following format:

(Device Number Populating Database) $\$ (Probe Device Number)

or

(Device Number Populating Database) $\triangleleft$ (Probe Device Number)

The symbol " $\varangle$ " denotes control cases where both probe and biometric reference database contain ONLY contact captured images.

The symbol " $\triangleleft$ " denotes all other cases (can be a mix of contactless vs contact, or contactless vs contactless) 


\subsection{Objective 1: Baseline (Contact Database to Contact Probes) Accuracy on Ten-Print and Mobile ID Matchers at Operational Thresholds.}

\begin{tabular}{ll}
\hline Biometric Reference Database Composition ${ }^{4}$ : & D1, both encounters, 8 fingers, full size \\
\hline Probe Set Composition: & D1, both encounters, 8 fingers, full size \\
\hline Matching Threshold: & As defined in section 4.9 for Ten-Print caseloads. \\
\hline Special Notes: & Represents baseline/control case \\
\hline Number of test trials in this Objective: & 4 for Ten-Print, and 4 for Mobile ID \\
\hline
\end{tabular}

The first objective of this study set out to establish how the matcher performs at a baseline level, and determine if the behavior of the matcher fits a normal distribution so that an appropriate selection of statistical tests can be applied.

For this objective, only images from the two encounters with optical/FTIR Appendix F certified Device 1 (D1) were utilized. To ensure the matcher behavior adheres to the symmetric property, each encounter was tested as the biometric reference database and the probe alternately.

This establishes baseline matcher performance and will be inclusive of any bias that may be present in the data selected for this study.

The thresholds used for this objective are analogous to the thresholds used in Ten-Print and Mobile ID configurations respectively.

\subsubsection{Findings for Objective 1}

When configured for Ten-Print mode of operation, the matcher achieved an FNIR of $0.5 \%$ at the low confidence threshold (yellow) and $0.5 \%$ at the high confidence/lights-out threshold (red) set in this study (see Table 5).

When configured for Mobile ID mode of operation, the matcher achieved an FNIR of $0.0 \%$ at the low confidence threshold (yellow) and $0.0 \%$ at the high confidence/lights-out threshold (red) set in this study (see Table 6).

For both test trials, the biometric reference database of D1-E1 (Device 1 Encounter 1) took longer on average to search as compared to using D1-E2 (Device 1 Encounter 2) as the biometric reference database (approximately $15 \%$ longer), measured on both the Mobile ID and Ten-Print matchers.

Examination of the distribution of both the matching score data as well as matcher service times in the normal probability plot [3] and the Q-Q Plot for this objective in Figure 3 shows that it is not possible to determine the distribution of the data and therefore parametric analysis methods are not suitable. Because of this, further analysis of data in this study will utilize non-parametric statistical methods.

\footnotetext{
4 The NIST Test Bed also contained 3 million records in addition to the test case being examined. The composition of the test bed biometric reference database is described in section 4.4 .
} 


\subsubsection{Data for Objective 1}

Table 5 - Baseline Ten-Print Matcher Performance

\begin{tabular}{|c|c|c|c|c|}
\hline \multirow{2}{*}{ Database } & \multirow{2}{*}{ Probe } & \multicolumn{2}{|c|}{ FNIR (\%) } & \multirow{2}{*}{ Average Search Time, ms } \\
\hline & & $\mathrm{T}=8500$ & $\mathrm{~T}=15000$ & \\
\hline D1-E1 & D1-E2 & 0.5 & 0.5 & 36105 \\
\hline D1-E2 & D1-E1 & 0.5 & 0.5 & 30600 \\
\hline
\end{tabular}

Table 6 - Baseline Mobile ID Matcher Performance

\begin{tabular}{ccccc}
\hline \multirow{2}{*}{ Database } & Probe & \multicolumn{2}{c}{ FNIR (\%) } & Average Search Time, ms \\
\cline { 3 - 4 } & & $\mathrm{T}=3000$ & $\mathrm{~T}=4100$ & \\
\hline D1-E1 & D1-E2 & 0 & 0 & 37506 \\
\hline D1-E2 & D1-E1 & 0 & 0 & 31754 \\
\hline
\end{tabular}

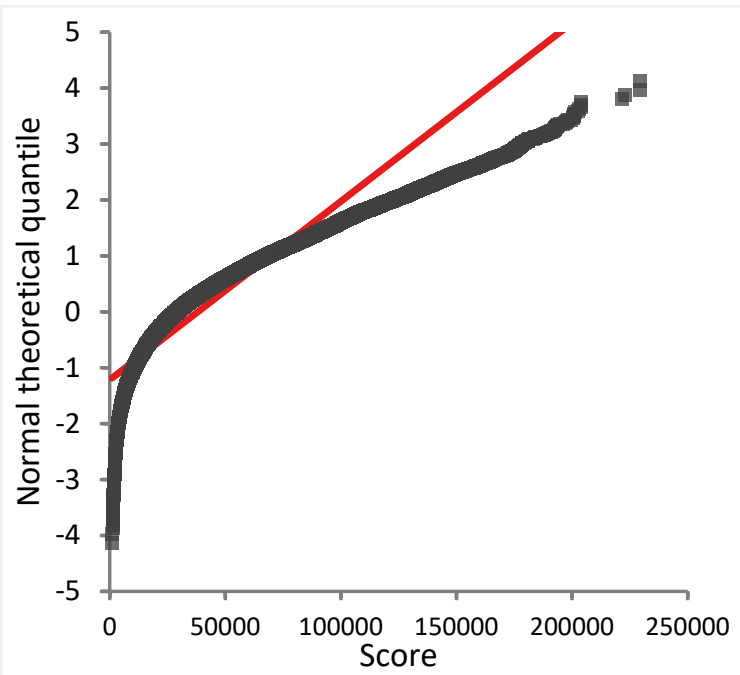

a. Ten-Print Matcher Score

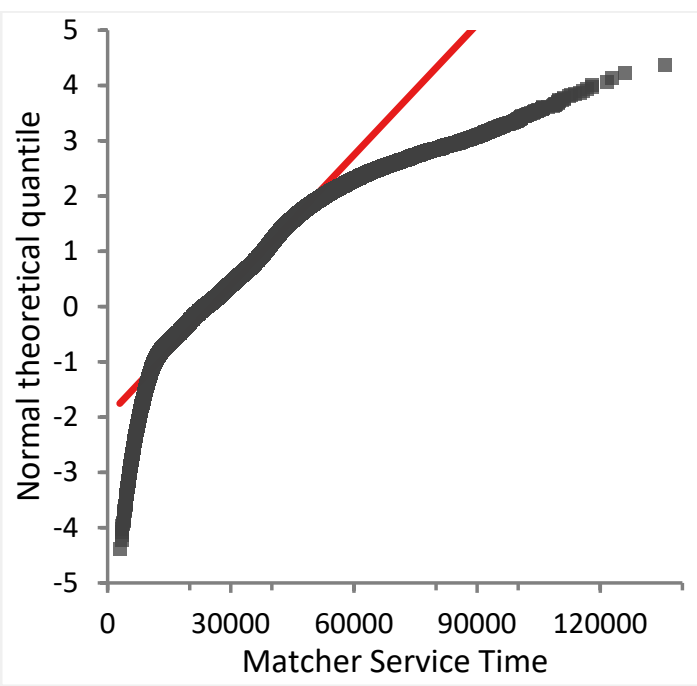

c. Ten-Print Matcher Service Time (ms)

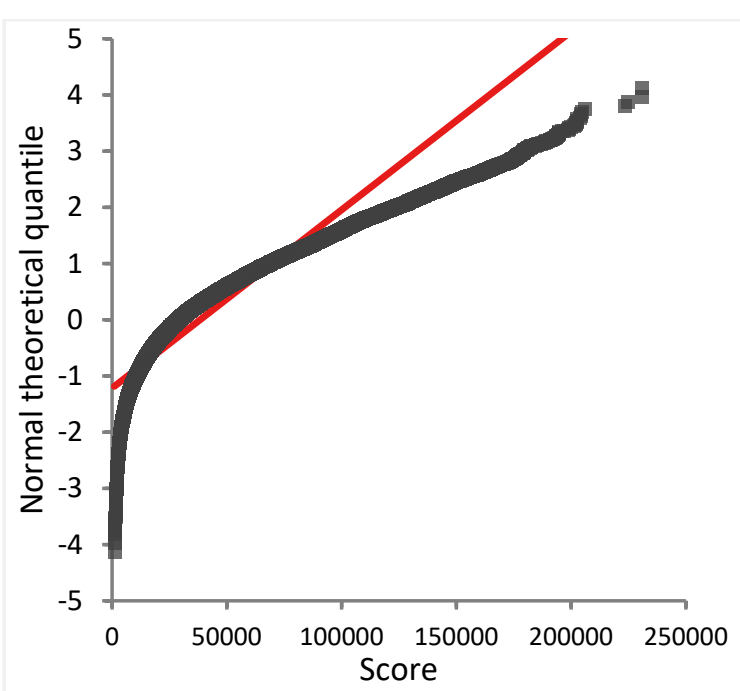

b. Mobile ID Matcher Score

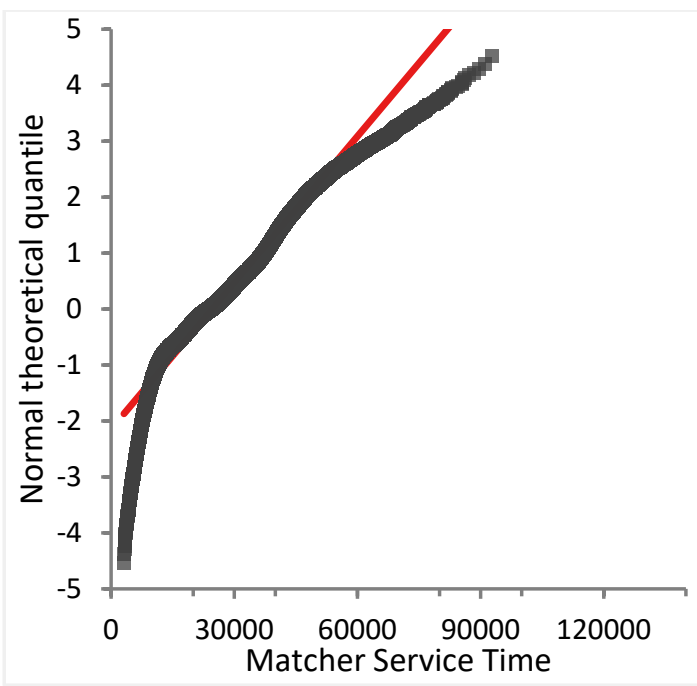

d. Mobile ID Matcher Service Time (ms)

Figure $3-Q-Q$ plots for match scores and matcher service times on the Ten-Print and Mobile ID matcher configurations. 


\subsection{Objective 2: Impact of Reduced Probe Finger Count on Accuracy at Operational Thresholds}

\begin{tabular}{ll}
\hline Biometric Reference Database Composition: & Two encounters for D1, and D2, full size, all 8 fingers \\
\hline Probe Set Composition: & All 8 devices, two encounters for D1, 8/6/4/2 fingers, full size \\
\hline Matching Threshold: & As defined in section 4.9 for Mobile ID and Ten-Print caseloads. \\
\hline Special Notes: & Also includes contact-to-contact cases for comparison \\
\hline Number of test trials in this Objective: & 192 (96 on Mobile ID, and 96 on Ten-Print) \\
\hline
\end{tabular}

In normal ten-print operation, the fingerprint matcher has the benefit of being able to operate on friction ridge information from all ten fingers presented (when available). Historically, systems have required ten-print capture to provide acceptable performance in terms of accuracy. As systems improved in capability, this allowed for matcher operation with transactions that provided the matcher less than the complete set of all ten fingers in their normal course of operation while at the same time providing performance that is functionally equivalent to full ten-print operation.

Given the challenges faced by some contactless devices in capturing all available prints, in this objective we measured the accuracy of both the Ten-Print and Mobile ID matching systems populated with contact collected images, and presented contactless probe transactions with reduced probe finger counts (from 8 fingers to 6,4 or even as few as 2 fingers).

\subsubsection{Findings for Objective 2}

Of the 96 test cases conducted on each matcher configuration, 24 of them were the control case (8 fingers or $8 \mathrm{~F}$ as denoted in the data tables). The remaining 72 represented a reduction of available fingers (to 6, 4 or 2 fingers).

Examination of score distributions for the Ten-Print matcher (Figure 4 and Table 7) and the Mobile ID matcher (Figure 5 and Table 9) show that the medians of the scores for each of the reduced finger count trials $(8,6,4$ and 2$)$ are different. This difference was confirmed to be statistically significant $(p<0.05)$ using the non-parametric Dwass-Steel-Critchlow-Fligner (DSCF) [4] test between all comparison pairs (Table 8 and Table 10).

Both the Ten-Print and Mobile ID matcher demonstrated resiliency with reduced finger counts for the control cases (contact to contact). The maximum error rate observed for the control cases was measured at 4.2\% FNIR for the 2 finger control case (D1-E2 4D2) on the Ten Print matcher (Table 11), and 0.5\% FNIR for the 4 finger cases (D1-E1 -D1-E2 and D1-E2 -D1-E1) on the Mobile ID matcher (Table 12).

Of the 54 non-control cases (not contact-to-contact cases) that represented finger count reduction on the Ten-Print matcher (Table 11) at operational threshold $\mathrm{T}=8500$, the FNIR stayed the same for 5 of these cases, increased with 42 of them, and surprisingly decreased (improved) with 7 (denoted with a " $\nabla$ " in the table), 6 of which represented $2 \mathrm{~F}$ submissions.

At operational threshold $\mathrm{T}=15000$, the Ten-Print matcher (Table 11) fared worse with the FNIR staying the same for 2 of these cases, increasing with 45 cases (worse), and decreasing (improving) with 7 cases (denoted with a " $\nabla$ " in the table), 4 of which represented $2 \mathrm{~F}$ submissions.

Of the 54 non-control cases (not contact-to-contact cases) cases that represented finger count reduction on the Mobile ID matcher (Table 12) at operational threshold $\mathrm{T}=3000$, the measured FNIR fared better than the Ten-Print system where 25 of the 54 cases stayed the same, 20 yielded increasing FNIR (worse), and again surprisingly 9 cases decreased (improved) (denoted with a " $\nabla$ " in the table), 5 of which represented $2 \mathrm{~F}$ submissions.

At operational threshold $\mathrm{T}=4100$, the Mobile ID matcher (Table 12) fared worse with the FNIR staying the same for 20 cases, increasing with 21 cases, and decreasing (improving) with 13 cases (denoted with a " $\nabla$ " in the table). 


\subsubsection{Data for Objective 2}

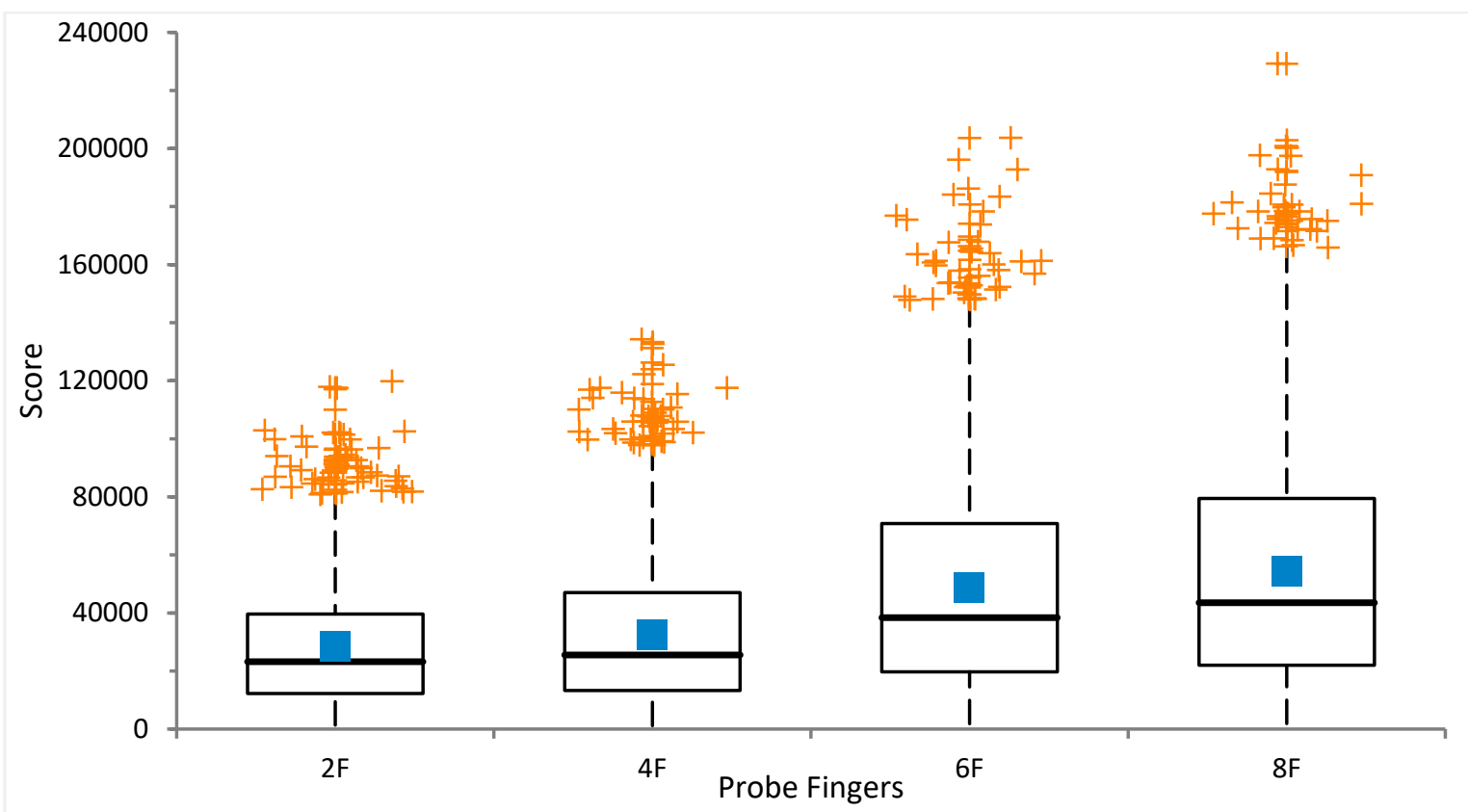

Figure 4 - Ten-Print Matcher Score Distribution by Probe Finger Count Using a Contact-Collected Biometric Reference Database.

Table 7 - Ten-Print Matcher Descriptive Score Data by Probe Finger Count Using a Contact-Collected Biometric Reference Database.

\begin{tabular}{|c|c|c|c|c|c|c|c|}
\hline $\begin{array}{l}\text { Probe } \\
\text { Finger } \\
\text { Count }\end{array}$ & Minimum & Mean & Median & Maximum & $\begin{array}{c}\text { 1st } \\
\text { Quartile }\end{array}$ & $\begin{array}{c}\text { 3rd } \\
\text { Quartile }\end{array}$ & IQR \\
\hline $2 F$ & 1352 & 28332.5 & 23204.5 & 119834 & 11402.2 & 39625.3 & 27368.3 \\
\hline $4 \mathrm{~F}$ & 1170 & 32507.5 & 25522.5 & 134292 & 12615.4 & 47001.8 & 33686.9 \\
\hline $6 F$ & 1838 & 48663.7 & 38380.0 & 203715 & 18029.0 & 70807.2 & 51065.7 \\
\hline $8 F$ & 1899 & 54290.3 & 43522.0 & 229254 & 20498.7 & 79467.3 & 57476.6 \\
\hline
\end{tabular}

Table 8 - DSCF Comparison of all Ten-Print Trials by Probe Finger Count Using a Contact-Collected Biometric Reference Database.

\begin{tabular}{r|r|r|r|r} 
Contrast & $\begin{array}{c}\text { Hodges- } \\
\text { Lehmann } \\
\text { location shift }\end{array}$ & Simultaneous $95 \% \mathrm{Cl}$ & 0 & p-value \\
\hline 8F $-2 \mathrm{~F}$ & 18496.0 & 16654.0 to 20396.0 & $<0.0001$ \\
$8 \mathrm{~F}-4 \mathrm{~F}$ & 15568.0 & 13743.0 to 17436.0 & $<0.0001$ \\
$8 \mathrm{~F}-6 \mathrm{~F}$ & 3955.0 & 2053.0 to 5879.0 & $<$ & $<0.0001$
\end{tabular}




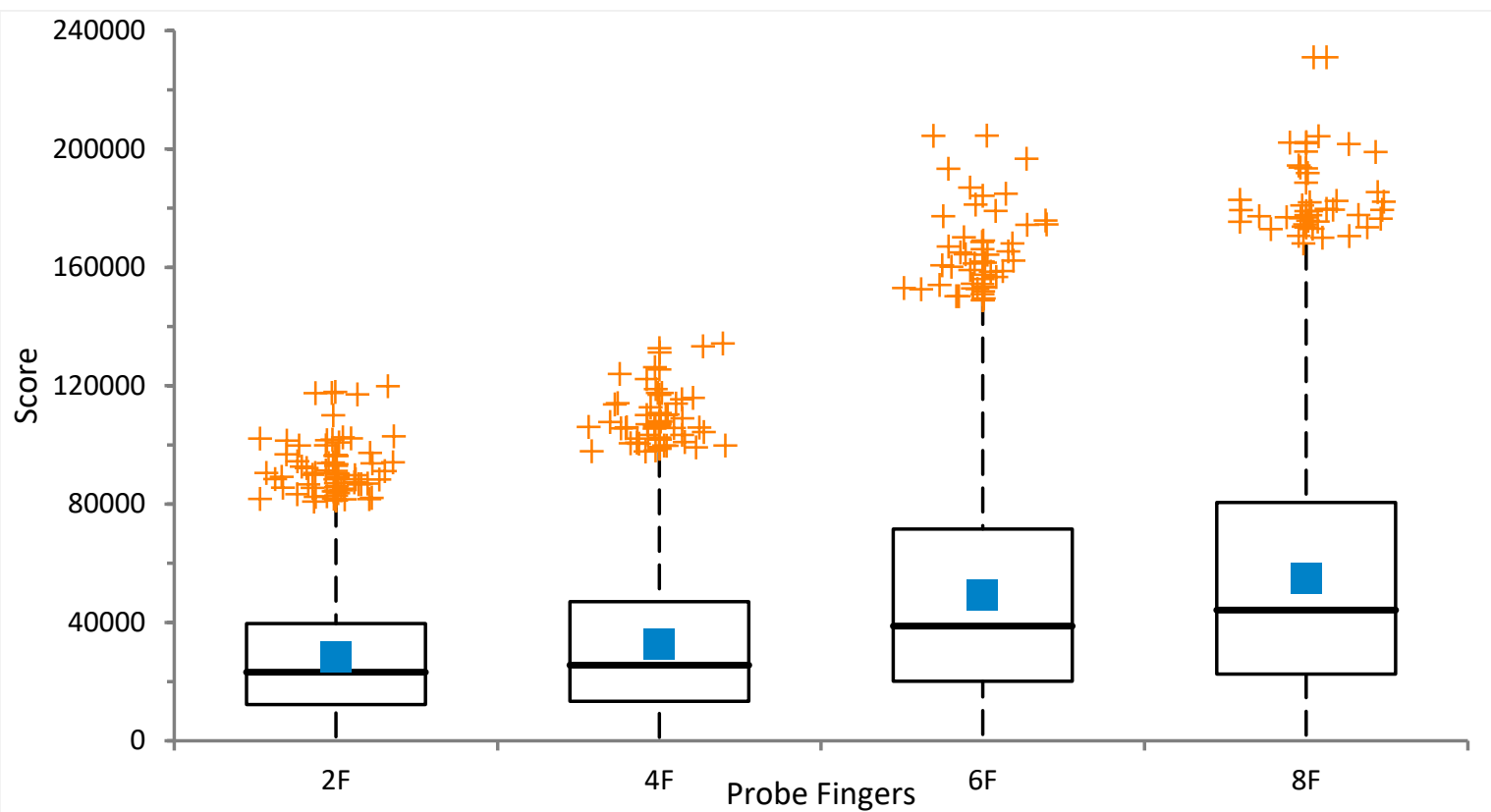

Figure 5 - Mobile ID Matcher Score Distribution by Probe Finger Count Using a Contact-Collected Biometric Reference Database.

Table 9 - Mobile ID Matcher Descriptive Score Data by Probe Finger Count Using a Contact-Collected Biometric Reference Database.

\begin{tabular}{r|c|cc|cc}
$\begin{array}{r}\text { Probe } \\
\text { Finger } \\
\text { Count }\end{array}$ & Mean & Median & $\begin{array}{c}\text { 1st } \\
\text { Quartile }\end{array}$ & $\begin{array}{c}\text { 3rd } \\
\text { Quartile }\end{array}$ & IQR \\
\hline $2 \mathrm{~F}$ & 28286.4 & 23177.0 & 12223.0 & 39590.8 & 27367.8 \\
$4 \mathrm{~F}$ & 32515.0 & 25558.0 & 13316.3 & 47010.3 & 33694.0 \\
$6 \mathrm{~F}$ & 49136.0 & 38770.0 & 20099.5 & 71579.5 & 51480.0 \\
$8 \mathrm{~F}$ & 54995.3 & 44171.5 & 22511.4 & 80543.0 & 58031.6
\end{tabular}

Table 10 - DSCF Comparison of all Mobile ID Trials by Probe Finger Count Using a Contact-Collected Biometric Reference Database.

\begin{tabular}{r|r|r|r|r} 
Contrast & $\begin{array}{c}\text { Hodges- } \\
\text { Lehmann } \\
\text { location shift }\end{array}$ & Simultaneous $95 \% \mathrm{Cl}$ & 0 & p-value \\
\hline $8 \mathrm{~F}-2 \mathrm{~F}$ & 19169.0 & 17312.0 to 21090.0 & $<0.0001$ \\
$8 \mathrm{~F}-4 \mathrm{~F}$ & 16181.0 & 14341.0 to 18067.0 & $<0.0001$ \\
$8 \mathrm{~F}-6 \mathrm{~F}$ & 4115.0 & 2188.0 to 6067.0 & $<0.0001$
\end{tabular}


Table 11 - Ten-Print Matcher Accuracy With Respect to Reduced Probe Finger Count at Operational Thresholds.

\begin{tabular}{|c|c|c|c|c|c|c|c|c|}
\hline Database (8F) & \multicolumn{3}{|c|}{ FNIR (\%), T=8500 } & $2 \mathrm{~F}$ & \multicolumn{3}{|c|}{ FNIR (\%), T=15000 } & $2 \mathrm{~F}$ \\
\hline $\mathrm{D} 1-\mathrm{E} 1<\mathrm{D} 2$ & 0 & 0 & 0 & 0 & 0 & 0 & 0 & 2.5 \\
\hline D1-E1 4D1-E2 & 0.5 & 0.5 & 0.5 & $0 \nabla$ & 0.5 & 0.5 & 0.5 & 0.5 \\
\hline D1-E2 4D1-E1 & 0.5 & 0.5 & 0.5 & $0 \nabla$ & 0.5 & 0.5 & 0.5 & 0.5 \\
\hline D2 $4 \mathrm{D} 1-\mathrm{E} 1$ & 0 & 0 & 0 & 0 & 0 & 0 & 0.8 & 3.3 \\
\hline $\mathrm{D} 1-\mathrm{E} 2<\mathrm{D} 2$ & 0 & 0 & 0 & 0 & 0 & 0 & 0 & 4.2 \\
\hline D2 4 D1-E2 & 0 & 0 & 0 & 0.8 & 0 & 0 & 0.8 & 3.3 \\
\hline $\mathrm{D} 2 \triangleleft \mathrm{D} 3$ & 0.8 & 0.8 & 0.8 & 2.5 & 1.6 & 3.3 & 7.6 & 8.4 \\
\hline $\mathrm{D} 1-\mathrm{E} 1 \triangleleft \mathrm{D} 3$ & 1.5 & 2.6 & 2.6 & 4.1 & 4.6 & $4.1 \nabla$ & 6.7 & 14 \\
\hline $\mathrm{D} 1-\mathrm{E} 2 \triangleleft \mathrm{D} 3$ & 2 & 2 & 3.1 & 4.1 & 5.2 & $4.1 \nabla$ & 7.8 & 15.6 \\
\hline $\mathrm{D} 1-\mathrm{E} 1 \triangleleft \mathrm{D} 4$ & 3.6 & $3.1 \nabla$ & 8.9 & 11.5 & 9.9 & 11.5 & 20.9 & 30.8 \\
\hline $\mathrm{D} 2 \triangleleft \mathrm{D} 4$ & 4.2 & 5.1 & 8.5 & $5.9 \nabla$ & 11.9 & 15.3 & 26.4 & 28.2 \\
\hline $\mathrm{D} 1-\mathrm{E} 2 \triangleleft \mathrm{D} 4$ & 3.1 & 3.6 & 5.7 & 12 & 10.4 & 12.5 & 23.5 & 35.6 \\
\hline $\mathrm{D} 1-\mathrm{E} 2 \triangleleft \mathrm{D} 8$ & 7.3 & 8.9 & 13 & 13 & 15.1 & 15.7 & 29.3 & 32.4 \\
\hline $\mathrm{D} 2 \triangleleft \mathrm{D} 8$ & 6.7 & 9.3 & 16.1 & $11 \nabla$ & 16.9 & $16.1 \nabla$ & 30.5 & 31.3 \\
\hline $\mathrm{D} 1-\mathrm{E} 1 \triangleleft \mathrm{D} 8$ & 8.9 & 9.4 & 12 & 13.6 & 16.7 & 18.3 & 29.8 & 32.9 \\
\hline $\mathrm{D} 2 \triangleleft \mathrm{D} 6$ & 1 & 3 & 12.2 & 15.3 & 11.2 & 14.2 & 45.9 & $42.8 \nabla$ \\
\hline D1-E1 $\triangleleft D 6$ & 3.7 & 5 & 11.9 & 15 & 15.7 & 18.8 & 40.8 & 47.7 \\
\hline D1-E2 $\triangleleft$ D6 & 4.4 & 6.2 & 13.2 & 13.8 & 15 & 18.8 & 44.6 & 44.6 \\
\hline $\mathrm{D} 2 \triangleleft \mathrm{D} 5$ & 9.2 & 14.2 & 25.2 & $17.6 \nabla$ & 24.3 & 29.4 & 47.8 & 49.5 \\
\hline D1-E1 $\triangleleft$ D5 & 11.9 & 12.9 & 20.7 & 26.4 & 24.3 & 31 & 50.7 & 50.7 \\
\hline D1-E2 $\triangleleft D 5$ & 11.9 & 13.9 & 22.2 & 23.8 & 29 & 33.6 & 51.2 & 53.8 \\
\hline $\mathrm{D} 2 \triangleleft \mathrm{D} 7$ & 12.6 & 16.8 & 34.4 & $31 \nabla$ & 36.1 & 42 & 52.9 & $47 \nabla$ \\
\hline D1-E1 $\triangleleft D 7$ & 19.2 & 20.8 & 36.9 & $27 \nabla$ & 40.1 & 43.7 & 57.8 & $49.4 \nabla$ \\
\hline D1-E2 $\triangleleft D 7$ & 18.7 & 22.3 & 38.5 & $27.6 \nabla$ & 39.5 & 44.2 & 59.8 & $46.3 \nabla$ \\
\hline
\end{tabular}

Table 12 - Mobile ID Matcher Accuracy With Respect to Reduced Probe Finger Count at Operational Thresholds.

\begin{tabular}{|c|c|c|c|c|c|c|c|c|}
\hline Database (8F) & $\begin{array}{c}\text { FNIR (\% } \\
8 \mathrm{~F}\end{array}$ & \multicolumn{2}{|c|}{ FNIR (\%), T=3000 } & $2 \mathrm{~F}$ & \multicolumn{3}{|c|}{ FNIR (\%), $T=4100$} & $2 \mathrm{~F}$ \\
\hline D1-E1 বD2 & 0 & 0 & 0 & 0 & 0 & 0 & 0 & 0 \\
\hline $\mathrm{D} 1-\mathrm{E} 2<\mathrm{D} 2$ & 0 & 0 & 0 & 0 & 0 & 0 & 0 & 0 \\
\hline $\mathrm{D} 2<\mathrm{D} 1-\mathrm{E} 1$ & 0 & 0 & 0 & 0 & 0 & 0 & 0 & 0 \\
\hline $\mathrm{D} 2<\mathrm{D} 1-\mathrm{E} 2$ & 0 & 0 & 0 & 0 & 0 & 0 & 0 & 0 \\
\hline $\mathrm{D} 2 \triangleleft \mathrm{D} 3$ & 0 & 0 & 0 & 0 & 0 & 0 & 0 & 0 \\
\hline $\mathrm{D} 1-\mathrm{E} 1<\mathrm{D} 1-\mathrm{E} 2$ & 0 & 0 & 0 & 0 & 0 & 0 & 0.5 & $0 \boldsymbol{\nabla}$ \\
\hline $\mathrm{D} 1-\mathrm{E} 2<\mathrm{D} 1-\mathrm{E} 1$ & 0 & 0 & 0 & 0 & 0 & 0 & 0.5 & $0 \nabla$ \\
\hline $\mathrm{D} 1-\mathrm{E} 1 \triangleleft \mathrm{D} 3$ & 0 & 0 & 0 & 0 & 0 & 0 & 0 & 1 \\
\hline $\mathrm{D} 1-\mathrm{E} 2 \triangleleft \mathrm{D} 3$ & 0 & 0 & 0.5 & 1 & 0 & 0 & 0.5 & 1.5 \\
\hline $\mathrm{D} 1-\mathrm{E} 1 \triangleleft \mathrm{D} 4$ & 0 & 0 & 0 & 1 & 0.5 & $0 \nabla$ & 1 & 1.5 \\
\hline $\mathrm{D} 1-\mathrm{E} 2 \triangleleft \mathrm{D} 6$ & 0.6 & 0.6 & 0.6 & 0.6 & 0.6 & 0.6 & 1.2 & 3.1 \\
\hline $\mathrm{D} 1-\mathrm{E} 2 \triangleleft \mathrm{D} 8$ & 0 & 0 & 0.5 & 0.5 & 2 & 2 & 2 & 2 \\
\hline $\mathrm{D} 2 \triangleleft \mathrm{D} 4$ & 0.8 & 0.8 & 0.8 & $0 \nabla$ & 1.7 & $0.8 \boldsymbol{\nabla}$ & 3.4 & $0.8 \nabla$ \\
\hline $\mathrm{D} 1-\mathrm{E} 1 \triangleleft \mathrm{D} 8$ & 0.5 & 0.5 & $0 \nabla$ & 0.5 & 1.5 & 2 & 2.6 & $2 \nabla$ \\
\hline $\mathrm{D} 1-\mathrm{E} 2 \triangleleft \mathrm{D} 4$ & 0 & 0 & 0.5 & 1 & 1.5 & $1 \nabla$ & 2 & 3.6 \\
\hline $\mathrm{D} 1-\mathrm{E} 1 \triangleleft \mathrm{D} 6$ & 0.6 & 1.2 & $0 \nabla$ & 1.2 & 1.8 & 1.8 & 1.8 & 1.8 \\
\hline $\mathrm{D} 2 \triangleleft \mathrm{D} 6$ & 1 & 1 & 1 & 2 & 1 & 1 & 2 & 2 \\
\hline $\mathrm{D} 1-\mathrm{E} 1 \triangleleft \mathrm{D} 5$ & 1 & 1.5 & $1 \nabla$ & $0.5 \boldsymbol{\nabla}$ & 3.1 & 3.1 & 3.1 & $1 \nabla$ \\
\hline $\mathrm{D} 2 \triangleleft \mathrm{D} 5$ & 0 & 0.8 & 0.8 & 0.8 & 2.5 & 3.3 & 5 & $1.6 \nabla$ \\
\hline $\mathrm{D} 2 \triangleleft \mathrm{D} 8$ & 0.8 & 1.6 & 2.5 & $1.6 \nabla$ & 1.6 & 2.5 & 4.2 & $1.6 \nabla$ \\
\hline $\mathrm{D} 1-\mathrm{E} 2 \triangleleft \mathrm{D} 5$ & 0.5 & 1 & 1.5 & $0.5 \mathrm{\nabla}$ & 2.5 & 2.5 & 5.6 & $3.1 \mathrm{~V}$ \\
\hline $\mathrm{D} 2 \triangleleft \mathrm{D} 7$ & 0.8 & 0.8 & 2.5 & 2.5 & 3.3 & $1.6 \nabla$ & 8.4 & 8.4 \\
\hline $\mathrm{D} 1-\mathrm{E} 1 \triangleleft \mathrm{D} 7$ & 2 & $1 \nabla$ & 1.5 & 3.1 & 3.6 & $3.1 \nabla$ & 10.4 & $7.2 \mathrm{~V}$ \\
\hline $\mathrm{D} 1-\mathrm{E} 2 \triangleleft \mathrm{D} 7$ & 1 & 1 & 5.2 & $3.1 \mathrm{\nabla}$ & 2.6 & 2.6 & 10.9 & $8.8 \nabla$ \\
\hline
\end{tabular}




\subsection{Objective 3: Impact of Reduced Probe Finger Count on Accuracy at Level Set Thresholds}

\begin{tabular}{ll}
\hline Biometric Reference Database Composition: & Two encounters for D1, and D2, full size, all 8 fingers \\
\hline Probe Set Composition: & All 8 devices, two encounters for D1, 8/6/4/2 Fingers, full size \\
\hline Matching Threshold: & As defined in section 4.9 for Mobile ID and Ten-Print caseloads. \\
\hline Special Notes: & Also includes contact-to-contact cases for comparison \\
\hline Number of test trials in this Objective: & 192 (96 on Mobile ID, and 96 on Ten-Print) \\
\hline
\end{tabular}

Like objective 2, in this objective we measure the accuracy of both the Ten-Print and Mobile ID matching systems populated using contact-collected biometric reference databases while operating on contactless probes with reduced finger counts (from 8 to 6, 4 or 2 fingers). Unlike objective 2 however, in this objective we measure accuracy on the Mobile ID and Ten-Print matchers operating at the same level set threshold.

\subsubsection{Findings for Objective 3}

Of the 96 test cases conducted on each matcher configuration, 24 of them were the control (8 fingers or $8 \mathrm{~F}$ as denoted in the data tables). The remaining 72 represented a reduction of available fingers (to 6 , 4 or 2 fingers). Given the DSCF statistical tests conducted for Objective 2, the determination that the treatments are different at $(\mathrm{p}<0.05)$ still holds and will not be repeated for this objective as the DSCF test results are not changed by altering error calculation thresholds.

Both the Ten-Print and Mobile ID matchers again showed resiliency with reduced finger counts for the control cases (contact-to-contact cases). The maximum error rate observed for the control cases (D1E1 $4 D 1-E 2, D 1-E 2 \varangle D 1-E 1, D 1-E 1<D 2, D 2 \varangle D 1-E 1, D 2 \varangle D 1-E 2)$ at the low threshold $T=5000$ was $0.5 \%$ for both matcher configurations (see Table 13 and Table 14) while the maximum measured FNIR for the high match threshold $\mathrm{T}=12000$ was $2.5 \%$ for both matchers.

A total of 24 device combinations was tested on the Ten-Print matcher. Of these 24 device combinations, 18 of these represented non-control combinations (not contact-to-contact) yielding 54 non-control cases. Of the 18 non-control device combinations examined, 7 of the 18 device combinations yielded monotonically increasing error rates as the number of probe fingers was reduced from $8 \mathrm{~F}$ at threshold $\mathrm{T}=5000$. The remaining 11 of 18 device combinations contained cases where the accuracy improved with finger count reduction (i.e., $\mathrm{D} 2 \triangleleft \mathrm{D} 8, \mathrm{~T}=5000,2 \mathrm{~F}, \mathrm{FNIR}=2.5 \%$ vs. $4 \mathrm{~F}$ with $\mathrm{FNIR}=5.0 \%$ ). These cases are denoted by a " $\nabla$ " in Table 13.

With the higher accuracy threshold of T=12000 on the Ten-Print matcher, of the 18 non-control device combinations tested, 13 of the 18 yielded monotonically increasing error rates as the number of probe fingers was reduced. The remaining 5 of 18 device combinations contained cases where the accuracy improved with finger count reduction.

A total of 18 non-control (not contact-to-contact) device combinations was tested on the Mobile ID matcher. Of the 18 combinations tested, 6 of the 18 yielded monotonically increasing error rates as the number of probe fingers was reduced from $8 \mathrm{~F}$ at threshold $\mathrm{T}=5000$ while the remaining 12 of 18 device combinations contained trials where the accuracy improved with finger count reduction. These cases where an improvement in accuracy was measured are denoted by a " $\boldsymbol{\nabla}$ " in Table 14 . This represents slightly better performance by the Mobile ID matcher versus the Ten-Print matcher at threshold T=5000 with reduced probe finger counts.

With the higher accuracy threshold of $\mathrm{T}=12000$ the Mobile ID matcher performed equally as well as the Ten-Print matcher, where 13 of the 18 non-control device combinations yielded monotonically increasing error rates as the number of probe fingers was reduced from $8 \mathrm{~F}$. The remaining 5 of 18 cases contained trials where the accuracy improved with finger count reduction. 
All in all, most cases examined on both matchers yielded increasing FNIR (worse match performance) with reduced probe finger counts. The Ten-Print matcher yielded 14 cases at threshold $\mathrm{T}=5000$ and 5 cases at threshold $\mathrm{T}=12000$ where the system showed better accuracy/lower FNIR with reduced finger counts. These results were slightly better on the Mobile ID matcher where it yielded 15 cases at threshold $\mathrm{T}=5000$ and 6 cases at threshold $\mathrm{T}=12000$ where FNIR was reduced by reducing probe finger counts.

Further analysis of the measured FNIR for each of the 192 test trials that comprised this objective showed that the relative difference in FNIR between the two matchers favors the Mobile ID configured matcher where 31 cases performed better than the Ten-Print matcher where 18 cases performed better (Table 15). 


\subsubsection{Data for Objective 3}

Table 13 - Ten-Print Matcher Accuracy With Respect to Reduced Probe Finger Count at Arbitrary Thresholds.

\begin{tabular}{|c|c|c|c|c|c|c|c|c|}
\hline \multirow[b]{2}{*}{ Database (8F) } & \multicolumn{4}{|c|}{ FNIR (\%), T=5000 } & \multicolumn{4}{|c|}{ FNIR (\%), T=12000 } \\
\hline & $8 \mathrm{~F}$ & $6 \mathrm{~F}$ & $4 \mathrm{~F}$ & $2 \mathrm{~F}$ & $8 \mathrm{~F}$ & $6 \mathrm{~F}$ & $4 \mathrm{~F}$ & $2 \mathrm{~F}$ \\
\hline D2 4D1-E1 & 0 & 0 & 0 & 0 & 0 & 0 & 0 & 0.8 \\
\hline $\mathrm{D} 1-\mathrm{E} 1<\mathrm{D} 2$ & 0 & 0 & 0 & 0 & 0 & 0 & 0 & 1.6 \\
\hline $\mathrm{D} 2<\mathrm{D} 1-\mathrm{E} 2$ & 0 & 0 & 0 & 0 & 0 & 0 & 0 & 1.6 \\
\hline $\mathrm{D} 1-\mathrm{E} 2<\mathrm{D} 2$ & 0 & 0 & 0 & 0 & 0 & 0 & 0 & 2.5 \\
\hline $\mathrm{D} 1-\mathrm{E} 1<\mathrm{D} 1-\mathrm{E} 2$ & 0.5 & 0.5 & 0.5 & $0 \boldsymbol{\nabla}$ & 0.5 & 0.5 & 0.5 & $0 \nabla$ \\
\hline D1-E2 4D1-E1 & 0.5 & 0.5 & 0.5 & $0 \boldsymbol{\nabla}$ & 0.5 & 0.5 & 0.5 & $0 \nabla$ \\
\hline $\mathrm{D} 2 \triangleleft \mathrm{D} 3$ & 0 & 0 & 0 & 0 & 0.8 & 1.6 & 4.2 & 4.2 \\
\hline $\mathrm{D} 1-\mathrm{E} 1 \triangleleft \mathrm{D} 3$ & 1 & 1 & $0 \nabla$ & 1 & 3.1 & 3.1 & 5.2 & 7.8 \\
\hline $\mathrm{D} 1-\mathrm{E} 2 \triangleleft \mathrm{D} 3$ & 0 & 0.5 & 1 & 2.6 & 3.6 & 3.6 & 6.7 & 9.3 \\
\hline $\mathrm{D} 1-\mathrm{E} 1 \triangleleft \mathrm{D} 4$ & 1.5 & $1 \nabla$ & 1.5 & 3.6 & 6.8 & 7.3 & 15.7 & 24.6 \\
\hline $\mathrm{D} 2 \triangleleft \mathrm{D} 4$ & 1.7 & 2.5 & 4.2 & $2.5 \nabla$ & 5.9 & 10.2 & 17.9 & 17.9 \\
\hline $\mathrm{D} 1-\mathrm{E} 2 \triangleleft \mathrm{D} 4$ & 2 & 2 & 2 & 4.7 & 5.7 & 7.8 & 16.7 & 24 \\
\hline $\mathrm{D} 2 \triangleleft \mathrm{D} 6$ & 1 & 1 & 3 & 3 & 3 & 5.1 & 28.5 & 28.5 \\
\hline $\mathrm{D} 2 \triangleleft \mathrm{D} 8$ & 2.5 & 3.3 & 5 & $2.5 \nabla$ & 11 & 13.5 & 22.8 & $20.3 \boldsymbol{\nabla}$ \\
\hline $\mathrm{D} 1-\mathrm{E} 2 \triangleleft \mathrm{D} 8$ & 2.6 & 2.6 & 3.1 & 5.2 & 10.9 & 13 & 19.8 & 25.1 \\
\hline $\mathrm{D} 1-\mathrm{E} 1 \triangleleft \mathrm{D} 8$ & 2 & 4.1 & 4.1 & $3.1 \nabla$ & 12.5 & 13 & 20.4 & 24.6 \\
\hline $\mathrm{D} 1-\mathrm{E} 2 \triangleleft \mathrm{D} 6$ & 1.2 & 1.2 & 3.1 & 5 & 8.1 & 9.4 & 26.4 & 29.5 \\
\hline $\mathrm{D} 1-\mathrm{E} 1 \triangleleft \mathrm{D} 6$ & 1.8 & 2.5 & 3.1 & 4.4 & 8.1 & 11.3 & 27.6 & 27.6 \\
\hline $\mathrm{D} 2 \triangleleft \mathrm{D} 5$ & 3.3 & 3.3 & 9.2 & $2.5 \nabla$ & 16.8 & 24.3 & 39.4 & $36.9 \nabla$ \\
\hline $\mathrm{D} 1-\mathrm{E} 1 \triangleleft \mathrm{D} 5$ & 3.6 & $3.1 \nabla$ & 8.2 & $5.1 \nabla$ & 17.6 & 22.2 & 38.3 & 42.4 \\
\hline $\mathrm{D} 1-\mathrm{E} 2 \triangleleft \mathrm{D} 5$ & 4.1 & $3.1 \mathrm{~V}$ & 8.8 & $7.2 \nabla$ & 19.6 & 25.9 & 37.8 & 43.5 \\
\hline $\mathrm{D} 2 \triangleleft \mathrm{D} 7$ & 4.2 & $3.3 \mathbf{\nabla}$ & 13.4 & $10 \nabla$ & 22.6 & 32.7 & 48.7 & $45.3 \nabla$ \\
\hline $\mathrm{D} 1-\mathrm{E} 2 \triangleleft \mathrm{D} 7$ & 4.6 & 7.2 & 14 & $12.5 \nabla$ & 27.6 & 33.3 & 53.6 & $41.6 \nabla$ \\
\hline $\mathrm{D} 1-\mathrm{E} 1 \triangleleft \mathrm{D} 7$ & 5.7 & 7.2 & 14 & $10.9 \nabla$ & 30.7 & 31.7 & 50 & $44.7 \nabla$ \\
\hline
\end{tabular}

Table 14 - Mobile ID Matcher Accuracy With Respect to Reduced Probe Finger Count at Arbitrary Thresholds.

\begin{tabular}{|c|c|c|c|c|c|c|c|c|}
\hline \multirow[b]{2}{*}{ Database (8F) } & \multicolumn{4}{|c|}{ FNIR (\%), T=5000 } & \multicolumn{4}{|c|}{ FNIR (\%), T=12000 } \\
\hline & $8 \mathrm{~F}$ & $6 \mathrm{~F}$ & $4 \mathrm{~F}$ & $2 \mathrm{~F}$ & $8 \mathrm{~F}$ & $6 \mathrm{~F}$ & $4 \mathrm{~F}$ & $2 \mathrm{~F}$ \\
\hline $\mathrm{D} 2<\mathrm{D} 1-\mathrm{E} 1$ & 0 & 0 & 0 & 0 & 0 & 0 & 0 & 0.8 \\
\hline $\mathrm{D} 1-\mathrm{E} 1<\mathrm{D} 2$ & 0 & 0 & 0 & 0 & 0 & 0 & 0 & 1.6 \\
\hline $\mathrm{D} 2<\mathrm{D} 1-\mathrm{E} 2$ & 0 & 0 & 0 & 0 & 0 & 0 & 0 & 1.6 \\
\hline $\mathrm{D} 1-\mathrm{E} 2<\mathrm{D} 2$ & 0 & 0 & 0 & 0 & 0 & 0 & 0 & 2.5 \\
\hline $\mathrm{D} 1-\mathrm{E} 1<\mathrm{D} 1-\mathrm{E} 2$ & 0.5 & 0.5 & 0.5 & $0 \boldsymbol{\nabla}$ & 0.5 & 0.5 & 0.5 & $0 \nabla$ \\
\hline D1-E2 $4 \mathrm{D} 1-\mathrm{E} 1$ & 0.5 & 0.5 & 0.5 & $0 \boldsymbol{\nabla}$ & 0.5 & 0.5 & 0.5 & $0 \nabla$ \\
\hline $\mathrm{D} 2 \triangleleft \mathrm{D} 3$ & 0 & 0 & 0 & 0 & 0.8 & 1.6 & 4.2 & 4.2 \\
\hline $\mathrm{D} 1-\mathrm{E} 1 \triangleleft \mathrm{D} 3$ & 1 & 1 & $0 \nabla$ & 1 & 3.1 & 3.1 & 5.2 & 7.8 \\
\hline $\mathrm{D} 1-\mathrm{E} 2 \triangleleft \mathrm{D} 3$ & 0 & 0.5 & 1 & 2.6 & 3.1 & 3.6 & 6.7 & 9.3 \\
\hline $\mathrm{D} 1-\mathrm{E} 1 \triangleleft \mathrm{D} 4$ & 1 & $0.5 \boldsymbol{\nabla}$ & 1.5 & 3.6 & 5.7 & 6.8 & 15.7 & 24.6 \\
\hline $\mathrm{D} 2 \triangleleft \mathrm{D} 4$ & 1.7 & 1.7 & 4.2 & $2.5 \nabla$ & 5.9 & 10.2 & 17.9 & 17.9 \\
\hline $\mathrm{D} 1-\mathrm{E} 2 \triangleleft \mathrm{D} 4$ & 2.6 & 2.6 & $2 \nabla$ & 4.7 & 6.2 & 8.3 & 16.7 & 24 \\
\hline $\mathrm{D} 2 \triangleleft \mathrm{D} 6$ & 1 & 1 & 3 & 3 & 2 & 5.1 & 28.5 & 28.5 \\
\hline $\mathrm{D} 2 \triangleleft \mathrm{D} 8$ & 1.6 & 3.3 & 5 & $2.5 \nabla$ & 10.1 & 12.7 & 22.8 & $20.3 \nabla$ \\
\hline $\mathrm{D} 1-\mathrm{E} 2 \triangleleft \mathrm{D} 6$ & 0.6 & 0.6 & 3.1 & 5 & 7.5 & 8.8 & 26.4 & 29.5 \\
\hline $\mathrm{D} 1-\mathrm{E} 2 \triangleleft \mathrm{D} 8$ & 2.6 & 2.6 & 3.1 & 5.2 & 10.9 & 13 & 19.8 & 25.1 \\
\hline $\mathrm{D} 1-\mathrm{E} 1 \triangleleft \mathrm{D} 8$ & 2 & 4.1 & 4.1 & $3.1 \nabla$ & 12.5 & 12.5 & 20.4 & 25.1 \\
\hline $\mathrm{D} 1-\mathrm{E} 1 \triangleleft \mathrm{D} 6$ & 1.8 & 2.5 & 3.1 & 4.4 & 8.1 & 11.3 & 27.6 & 27.6 \\
\hline $\mathrm{D} 2 \triangleleft \mathrm{D} 5$ & 3.3 & 3.3 & 9.2 & $2.5 \nabla$ & 16.8 & 24.3 & 39.4 & $36.9 \nabla$ \\
\hline $\mathrm{D} 1-\mathrm{E} 1 \triangleleft \mathrm{D} 5$ & 4.1 & $3.6 \nabla$ & 7.2 & $5.1 \nabla$ & 17 & 22.2 & 37.3 & 42.4 \\
\hline $\mathrm{D} 1-\mathrm{E} 2 \triangleleft \mathrm{D} 5$ & 3.1 & 3.1 & 9.3 & $7.2 \nabla$ & 17 & 25.3 & 38.3 & 43.5 \\
\hline $\mathrm{D} 2 \triangleleft \mathrm{D} 7$ & 4.2 & $3.3 \boldsymbol{\nabla}$ & 13.4 & $10.9 \nabla$ & 21 & 29.4 & 49.5 & $47 \nabla$ \\
\hline $\mathrm{D} 1-\mathrm{E} 2 \triangleleft \mathrm{D} 7$ & 4.6 & 6.2 & 14.5 & $13 \boldsymbol{\nabla}$ & 24.4 & 29.1 & 53.1 & $42.7 \nabla$ \\
\hline $\mathrm{D} 1-\mathrm{E} 1 \triangleleft \mathrm{D} 7$ & 6.2 & $5.7 \boldsymbol{\nabla}$ & 13.5 & $11.4 \boldsymbol{\nabla}$ & 29.6 & $29.1 \nabla$ & 50 & $45.8 \nabla$ \\
\hline
\end{tabular}


Table 15 -Ten-Print vs. Mobile ID Matcher Performance Delta at Arbitrary Threshold With Respect to Reduced Probe Finger Count.

\begin{tabular}{|c|c|c|c|c|c|c|c|c|}
\hline \multirow[b]{2}{*}{ Database (8F) } & \multicolumn{4}{|c|}{ FNIR ( $\Delta \%$ Ten-Print vs Mobile ID), $T=5000$} & \multicolumn{4}{|c|}{$=\operatorname{NIR}(\Delta \%$ Ten-Print vs Mobile ID), T=12000 } \\
\hline & $8 \mathrm{~F}$ & $6 \mathrm{~F}$ & $4 \mathrm{~F}$ & $2 \mathrm{~F}$ & $8 \mathrm{~F}$ & $6 \mathrm{~F}$ & $4 \mathrm{~F}$ & $2 \mathrm{~F}$ \\
\hline $\mathrm{D} 1-\mathrm{E} 2 \triangleleft \mathrm{D} 4$ & -0.6 & -0.6 & 0 & 0 & -0.5 & -0.5 & 0 & 0 \\
\hline D1-E1 4D1-E2 & 0 & 0 & 0 & 0 & 0 & 0 & 0 & 0 \\
\hline $\mathrm{D} 1-\mathrm{E} 1<\mathrm{D} 2$ & 0 & 0 & 0 & 0 & 0 & 0 & 0 & 0 \\
\hline $\mathrm{D} 1-\mathrm{E} 1 \triangleleft \mathrm{D} 3$ & 0 & 0 & 0 & 0 & 0 & 0 & 0 & 0 \\
\hline D1-E1 $ব D 6$ & 0 & 0 & 0 & 0 & 0 & 0 & 0 & 0 \\
\hline $\mathrm{D} 1-\mathrm{E} 1 \triangleleft \mathrm{D} 8$ & 0 & 0 & 0 & 0 & 0 & 0.5 & 0 & -0.5 \\
\hline D1-E2 4D1-E1 & 0 & 0 & 0 & 0 & 0 & 0 & 0 & 0 \\
\hline $\mathrm{D} 1-\mathrm{E} 2<\mathrm{D} 2$ & 0 & 0 & 0 & 0 & 0 & 0 & 0 & 0 \\
\hline $\mathrm{D} 1-\mathrm{E} 2 \triangleleft \mathrm{D} 8$ & 0 & 0 & 0 & 0 & 0 & 0 & 0 & 0 \\
\hline D2 $4 \mathrm{D} 1-\mathrm{E} 1$ & 0 & 0 & 0 & 0 & 0 & 0 & 0 & 0 \\
\hline $\mathrm{D} 2<\mathrm{D} 1-\mathrm{E} 2$ & 0 & 0 & 0 & 0 & 0 & 0 & 0 & 0 \\
\hline $\mathrm{D} 2 \triangleleft \mathrm{D} 3$ & 0 & 0 & 0 & 0 & 0 & 0 & 0 & 0 \\
\hline $\mathrm{D} 2 \triangleleft \mathrm{D} 5$ & 0 & 0 & 0 & 0 & 0 & 0 & 0 & 0 \\
\hline $\mathrm{D} 1-\mathrm{E} 2 \triangleleft \mathrm{D} 3$ & 0 & 0 & 0 & 0 & 0.5 & 0 & 0 & 0 \\
\hline $\mathrm{D} 2 \triangleleft \mathrm{D} 4$ & 0 & 0.8 & 0 & 0 & 0 & 0 & 0 & 0 \\
\hline $\mathrm{D} 2 \triangleleft \mathrm{D} 6$ & 0 & 0 & 0 & 0 & 1 & 0 & 0 & 0 \\
\hline $\mathrm{D} 2 \triangleleft \mathrm{D} 7$ & 0 & 0 & 0 & -0.9 & 1.6 & 3.3 & -0.8 & -1.7 \\
\hline D1-E1 বD5 & -0.5 & -0.5 & 1 & 0 & 0.6 & 0 & 1 & 0 \\
\hline D1-E2 $\triangleleft$ D6 & 0.6 & 0.6 & 0 & 0 & 0.6 & 0.6 & 0 & 0 \\
\hline D1-E1 $\triangleleft \mathrm{D} 4$ & 0.5 & 0.5 & 0 & 0 & 1.1 & 0.5 & 0 & 0 \\
\hline $\mathrm{D} 2 \triangleleft \mathrm{D} 8$ & 0.9 & 0 & 0 & 0 & 0.9 & 0.8 & 0 & 0 \\
\hline D1-E2 $\triangleleft$ D5 & 1 & 0 & -0.5 & 0 & 2.6 & 0.6 & -0.5 & 0 \\
\hline D1-E1 $ব D 7$ & -0.5 & 1.5 & 0.5 & -0.5 & 1.1 & 2.6 & 0 & -1.1 \\
\hline D1-E2 $\triangleleft D 7$ & 0 & 1 & -0.5 & -0.5 & 3.2 & 4.2 & 0.5 & -1.1 \\
\hline
\end{tabular}

Mobile ID Matcher Performs Better (31 cases)

Ten-Print Matcher Performs Better (18 cases) 


\subsection{Objective 4: Impact of Probe Image Size Reduction on Accuracy at Level Set Thresholds}

\begin{tabular}{ll}
\hline Biometric Reference Database Composition: & Two encounters for D1, and D2, 8F, FAP40/30/20/10 \\
\hline Probe Set Composition: & All 8 devices, two encounters for D1, 8F, FAP40/30/20/10 \\
Matching Threshold: & As defined in section 4.9 for Ten-Print caseloads. \\
$\begin{array}{l}\text { Special Notes: } \\
\text { Number of test trials in this Objective: }\end{array}$ & $\begin{array}{l}\text { Also includes contact-to-contact cases for comparison } \\
\text { each })\end{array}$ \\
\hline
\end{tabular}

An important aspect of fingerprint matching is to provide the system with as much of the friction ridge surface area as possible thereby improving its chances of finding an overlapping constellation of features in establishing a match. As matcher technology and computational ability improved over the years, matchers have been able to do more with less in terms of the friction ridge surface area which ushered in advances such as Mobile ID [12]. Mobile ID allowed for the introduction of a new generation fingerprint capture devices that provide for portable in-the-field capture of fingerprints, albeit with a smaller capture area. The Mobile ID provisions of the ANSI/NIST standard [2] allow participating stakeholders to accept these smaller images into their processing pathway. While this reduction in capture size may result in diminished system matching ability, on a modern system this may be an acceptable tradeoff of in-the-scene processing of individuals.

Anecdotal evidence and observations at the time of data collection for this study showed that while contactless capture devices may also share some of the limitations of Mobile ID capture devices where the friction ridge capture may be smaller than traditional contact capture devices, the contactless devices may face another issue where unconstrained capture in a 3D volumetric space may lead to capturing superfluous data from around the fingerprint including background, fingernails, as well as possible distortion due to depth of field challenges in photographic capture of the fingerprint.

This objective set out to explore utilizing the Mobile ID specified allowable FAPs to trim and remove some of the image perimeter data from the contactless captured fingerprints, and then observe the impact of this trimming on matcher accuracy.

Probe images were prepared by first calculating centroid for the segmented fingerprint image, and then centering a trimming box with the desired FAP dimensions according to the various mobile ID specifications (for FAP40, FAP30, FAP20 and FAP10) around that centroid, and trimming the image. While the centroid detection was facilitated by automated tools, manual verification and correction of centroid placement was conducted on the images prior to trimming. Cases corresponding to these images were then processed on both the Mobile optimized matcher as well as the ten-print optimized matcher at the same arbitrary level-set matching threshold, with no other changes to the data to favor either system. Also for the purposes of analysis in this study, FAP40 (the largest image) is defined as the control.

\subsubsection{Findings for Objective 4}

There were three treatments (trimming images from FAP40-to-FAP30, FAP40-to-FAP20 and FAP40to-FAP10), each of these treatments generated 72 cases for a total of 216 test cases processed on each matcher/capture device configuration.

Examination of score distributions for the Ten-Print matcher (Figure 6 and Table 16) and the Mobile ID matcher (Figure 7 and Table 18) show that the medians of the scores for each of the image size treatments FAP40, FAP30, FAP20 and FAP10 are different. This difference was confirmed to be statistically significant $(\mathrm{p}<0.001)$ using the DSCF test for the cases of FAP40-to-FAP20 and FAP40-toFAP10 (Table 17 and Table 19) on both the Ten-Print and Mobile ID configurations. The distribution of scores between case of FAP40-to-FAP30 and the control case FAP40 however was not deemed different using DSCF for the Ten-Print matcher $(\mathrm{p}=0.9992)$ and the Mobile ID matcher $(\mathrm{p}=1.000)$ and results from the FAP40-to-FAP30 treatment are anecdotal and may be inconclusive.

Next, accuracy measurements (FNIR) for all test trials (Table 20) showed that generally larger images (FAP40) yielded lower FNIR on the both Ten-Print and Mobile ID matchers, but not universally so. 
Taking all trial cases and categorizing them by the three treatments FAP40-to-FAP30, FAP40-to-FAP20 and FAP40-to-FAP10, the treatments generally yielded more degraded cases (in terms of match accuracy) than improvements (Figure 8, Figure 9 and Table 21). The treatment of FAP40-to-FAP20 at threshold $\mathrm{T}=5000$ had an unexpected net positive effect where 7 of the cases degraded in accuracy but 9 improved (Table 21).

Further examination of FNIR performance by capture device (Table 22 and Table 23) shows a more telling story in this expected finding where it can be seen that certain devices perform markedly better across all test cases with images that are cropped rather than in their original size. For example, probes generated by D4 and D6 yield better performance on both matcher configurations, at both thresholds $\mathrm{T}=5000$ and $\mathrm{T}=12000$ at the treatment FAP40-to-FAP30. While noted above that the distribution of scores between case of (FAP40-to-FAP30) and the control case (FAP40) was not statistically significant on the Ten-Print matcher $(p=0.9992)$ and the Mobile ID matcher $(p=1.000)$, the specific cases of D4-FAP40-to-FAP30 and D6-FAP40-to-FAP30 versus control D1-E1 are at $(\mathrm{p}<0.05)$ for both the Ten-Print matcher (Figure 10 and Table 24 and the Mobile ID matcher (Figure 11 and Table 25 ) at thresholds examined.

Improvements such as those observed by D4 and D6 were not isolated. Of the 288 cases examined, 57 of them showed improvements while 136 degraded, and 95 remained the same with respect to matching performance. 


\subsubsection{Data for Objective 4}

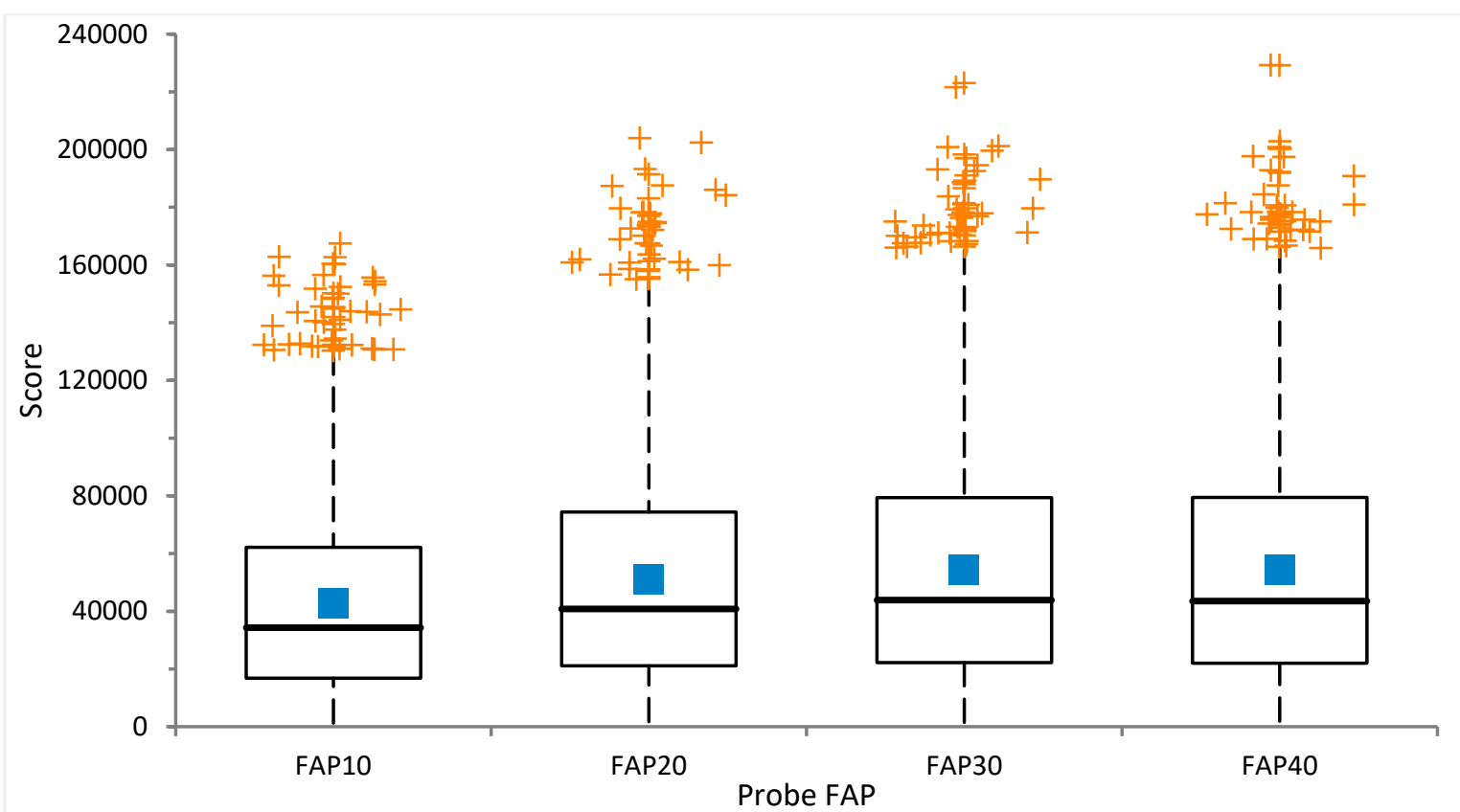

Figure 6 - Ten-Print Matcher Score Distribution by Probe FAP Size (vs. Contact-Collected Biometric Reference Database).

Table 16 - Ten-Print Matcher Descriptive Score Data by Probe FAP Size (vs. Contact-Collected Biometric Reference Database).

\begin{tabular}{r|r|rr|rr}
$\begin{array}{r}\text { Probe } \\
\text { FAP Size }\end{array}$ & \multicolumn{1}{|c|}{ Mean } & \multicolumn{1}{|c}{ Median } & 1st Quartile & 3rd Quartile & \multicolumn{1}{c}{ IQR } \\
\hline FAP10 & 42739.0 & 34305.0 & 16794.3 & 62173.0 & 45378.7 \\
FAP20 & 51125.5 & 40802.0 & 21104.0 & 74378.2 & 53274.2 \\
FAP30 & 54478.1 & 43871.0 & 22194.3 & 79373.0 & 57178.7 \\
FAP40 & 54290.3 & 43522.0 & 21990.8 & 79467.3 & 57476.6
\end{tabular}

Table 17 - DSCF Comparison of all Ten-Print Matcher Trials by Probe FAP Size (vs. Contact-Collected Biometric Reference Database).

\begin{tabular}{|c|c|c|c|c|}
\hline Contrast & $\begin{array}{c}\text { Hodges- } \\
\text { Lehmann } \\
\text { location shift }\end{array}$ & Simultaneous $95 \% \mathrm{Cl}$ & 0 & $\mathrm{p}$-value \\
\hline FAP40 - FAP10 & 8152.0 & 6280.0 to 10053.0 & & $<0.0001$ \\
\hline FAP40 - FAP20 & 2053.0 & 115.0 to 4015.0 & & 0.0329 \\
\hline FAP40 - FAP30 & 219.0 & -1772.0 to 2205.0 & & 0.9919 \\
\hline
\end{tabular}




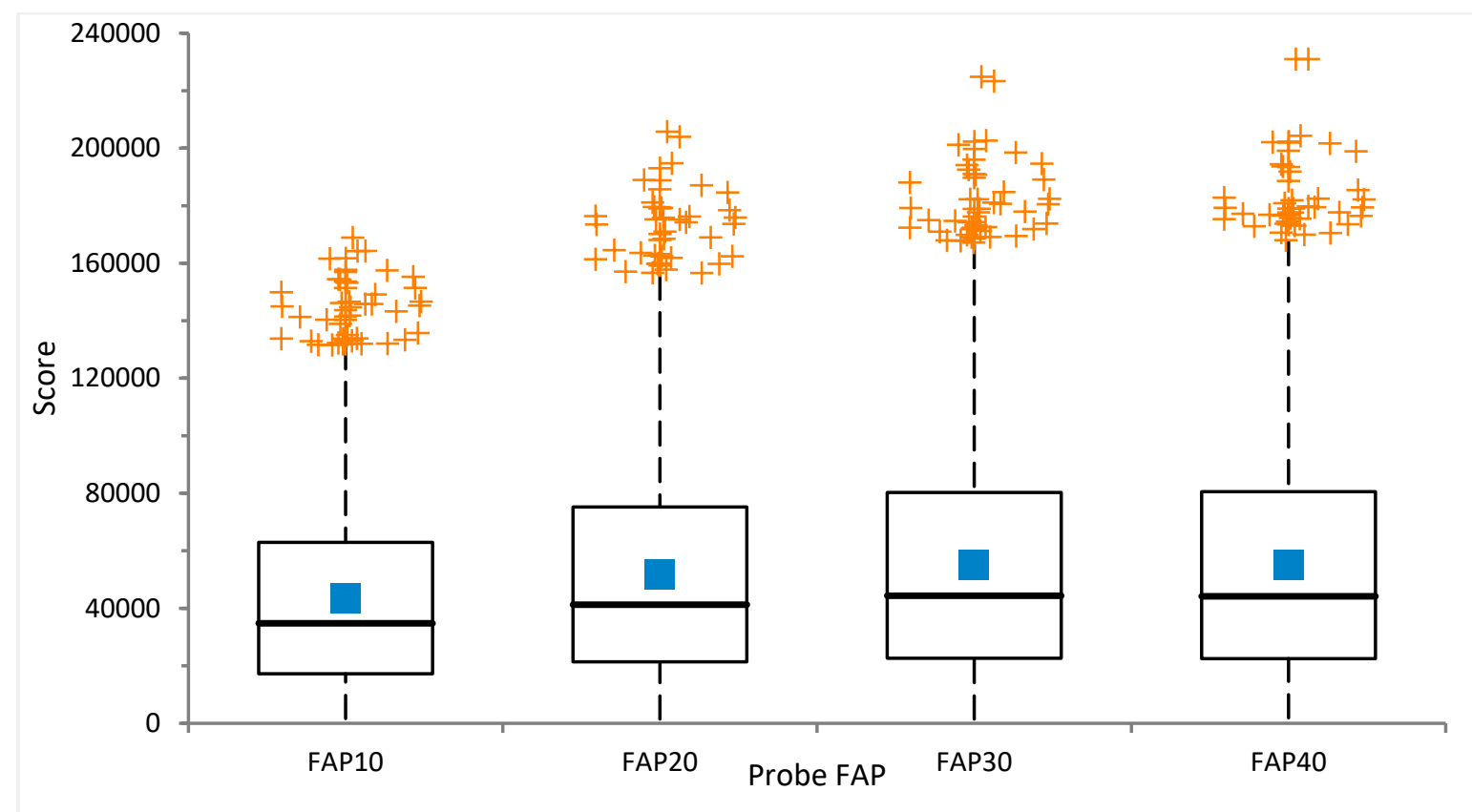

Figure 7 - Mobile ID Matcher Score Distribution by Probe FAP Size.

Table 18 - Mobile ID Matcher Descriptive Score Data by Probe FAP Size (vs. Contact-Collected Biometric Reference Database).

\begin{tabular}{|c|c|c|c|c|c|}
\hline $\begin{array}{c}\text { Probe } \\
\text { FAP }\end{array}$ & Mean & Median & 1st Quartile & 3rd Quartile & IQR \\
\hline FAP10 & 43305.1 & 34781.0 & 17226.8 & 62898.5 & 45671.7 \\
\hline FAP20 & 51686.6 & 41251.5 & 21374.8 & 75223.3 & 53848.5 \\
\hline FAP30 & 55075.5 & 44366.5 & 22632.8 & 80305.6 & 57672.8 \\
\hline FAP40 & 54995.3 & 44171.5 & 22511.4 & 80543.0 & 58031.6 \\
\hline
\end{tabular}

Table 19 - Comparison of Mobile ID Matcher Trials by Probe FAP Size (vs. Contact-Collected Biometric Reference Database).

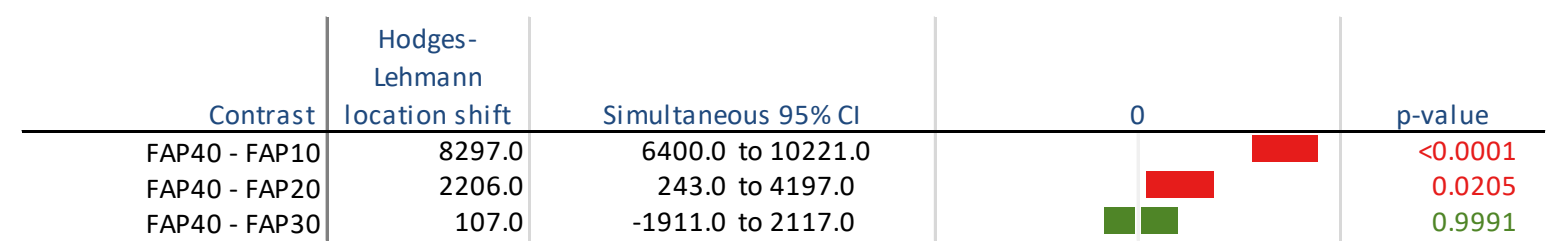


Table 20 - Error Differential Between Ten-Print and Mobile ID Matchers at Arbitrary Thresholds, by Size (FAP), Versus Control (FAP40).

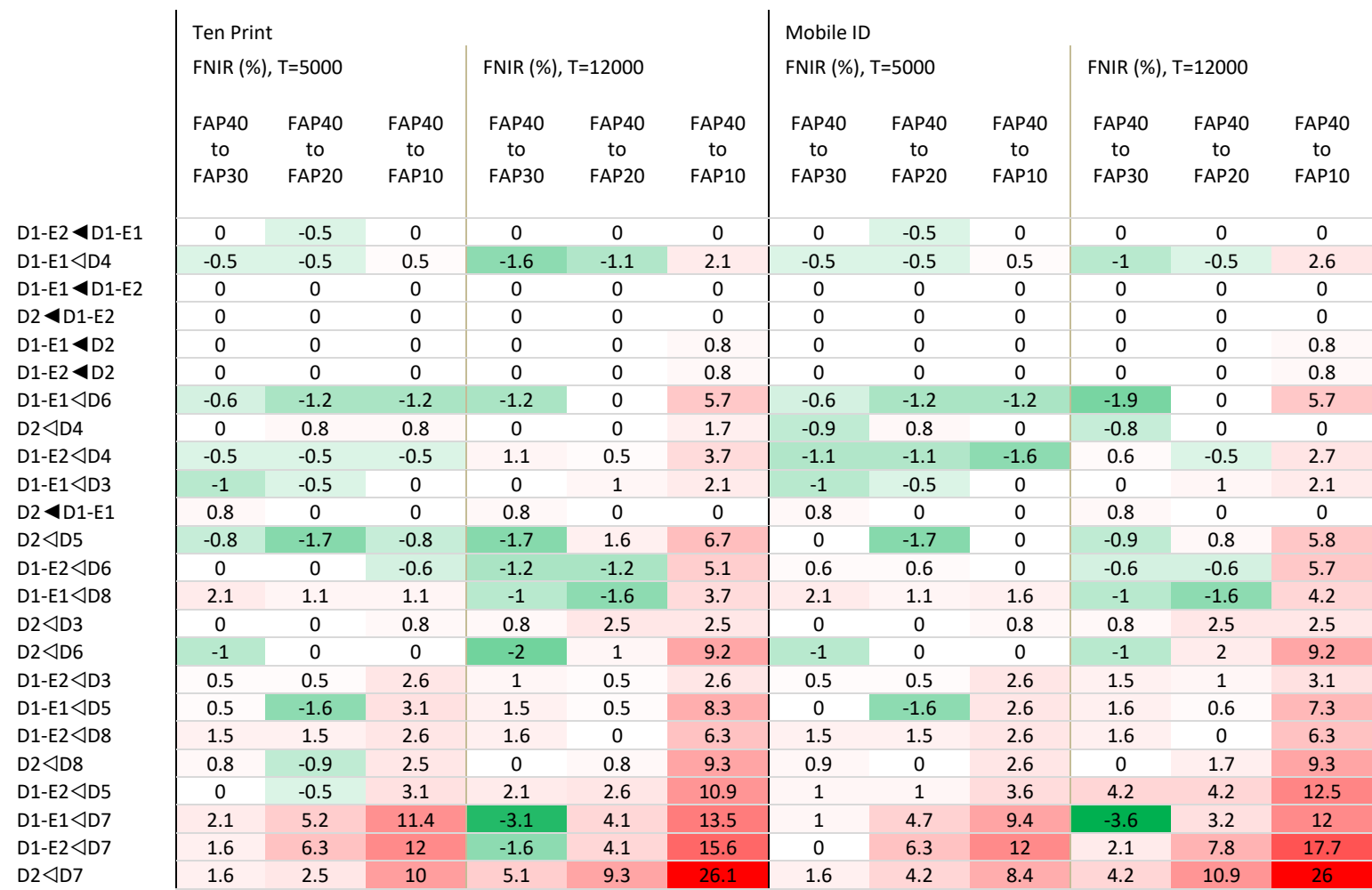

Legend:

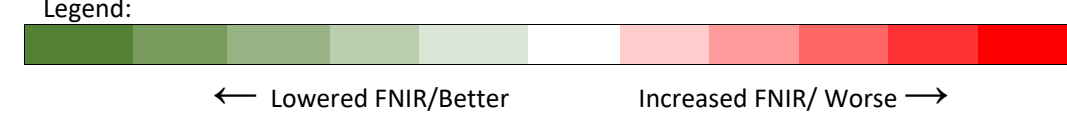




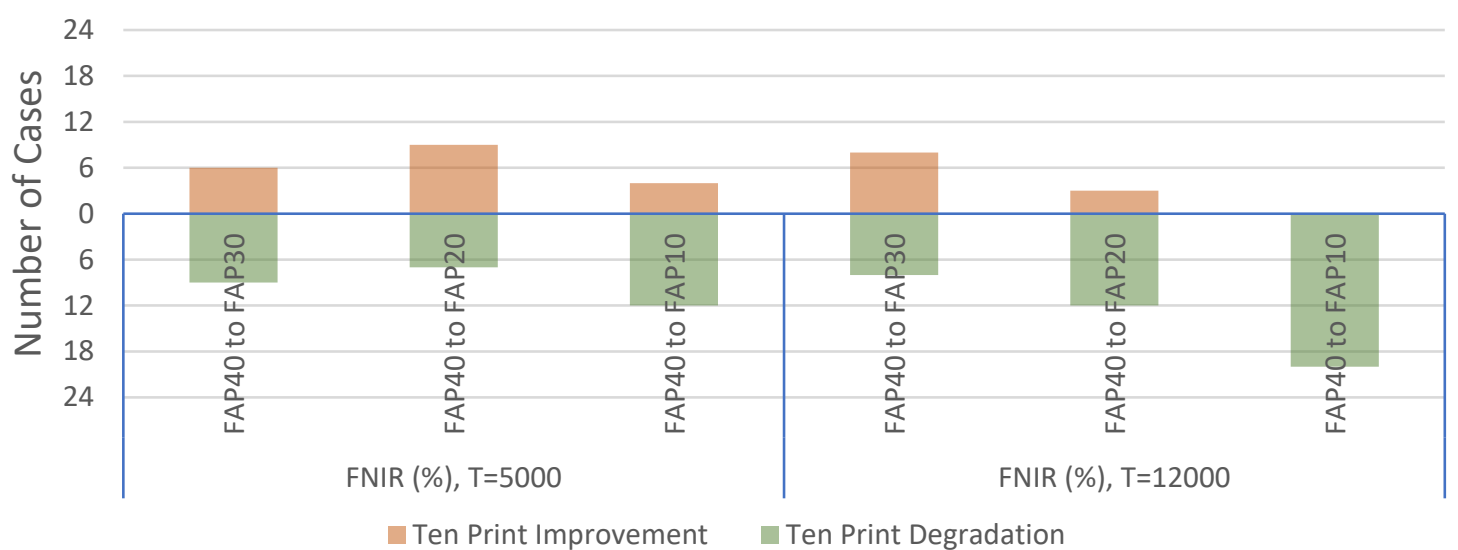

Figure 8 - Comparison Improved/Degraded Cases on the Ten-Print Matcher Using a Contact-Collected Biometric Reference Database Only, With Respect to Reduced Image Size by Trimming Original Image to Mobile ID FAP30, FAP20 and FAP10.

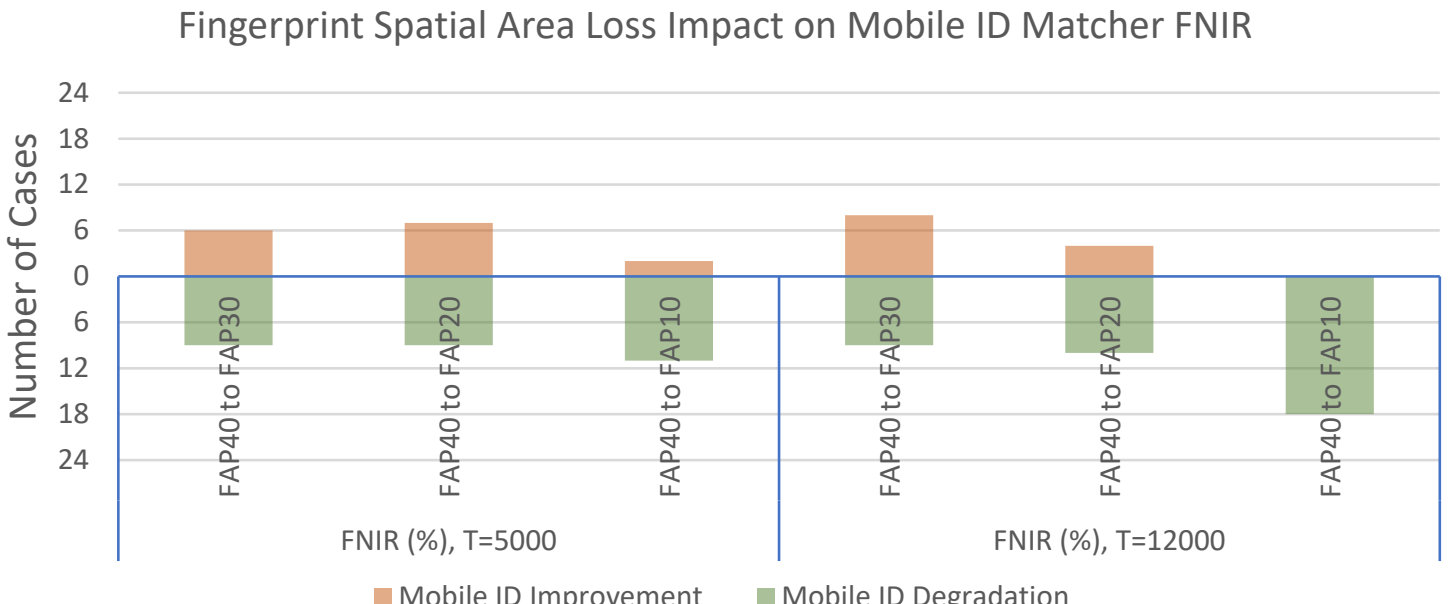

Figure 9 - Comparison Improved/Degraded Cases On The Mobile ID Matcher Using a Contact-Collected Biometric Reference Database Only, With Respect to Reduced Image Size by Trimming Original Image to Mobile ID FAP30, FAP20 And FAP10.

Table 21 - Data Table for Figure 3 and Figure 4.

\begin{tabular}{|l|c|c|c|c|c|c|}
\hline & \multicolumn{2}{|c|}{ FNIR (\%), T=5000 } & \multicolumn{2}{c|}{ FNIR (\%), T=12000 } \\
\hline & $\begin{array}{c}\text { FAP40 to } \\
\text { FAP30 }\end{array}$ & $\begin{array}{c}\text { FAP40 to } \\
\text { FAP20 }\end{array}$ & $\begin{array}{c}\text { FAP40 to } \\
\text { FAP10 }\end{array}$ & $\begin{array}{c}\text { FAP40 to } \\
\text { FAP30 }\end{array}$ & $\begin{array}{c}\text { FAP40 to } \\
\text { FAP20 }\end{array}$ & $\begin{array}{c}\text { FAP40 to } \\
\text { FAP10 }\end{array}$ \\
\hline Ten Print Improvement & 6 & $\mathbf{9}$ & 4 & 8 & 3 & 0 \\
\hline Ten Print Degradation & $\mathbf{9}$ & 7 & $\mathbf{1 2}$ & 8 & $\mathbf{1 2}$ & $\mathbf{2 0}$ \\
\hline Mobile ID Improvement & 6 & 7 & 2 & 8 & 4 & 0 \\
\hline Mobile ID Degradation & $\mathbf{9}$ & $\mathbf{9}$ & $\mathbf{1 1}$ & $\mathbf{9}$ & $\mathbf{1 0}$ & $\mathbf{1 8}$ \\
\hline
\end{tabular}


Table 22 - Count of Cases Where FNIR Improved (+) Or Degraded (-) via Image Cropping, by Probe Capture Device (vs Contact-Collected Biometric Reference Database).

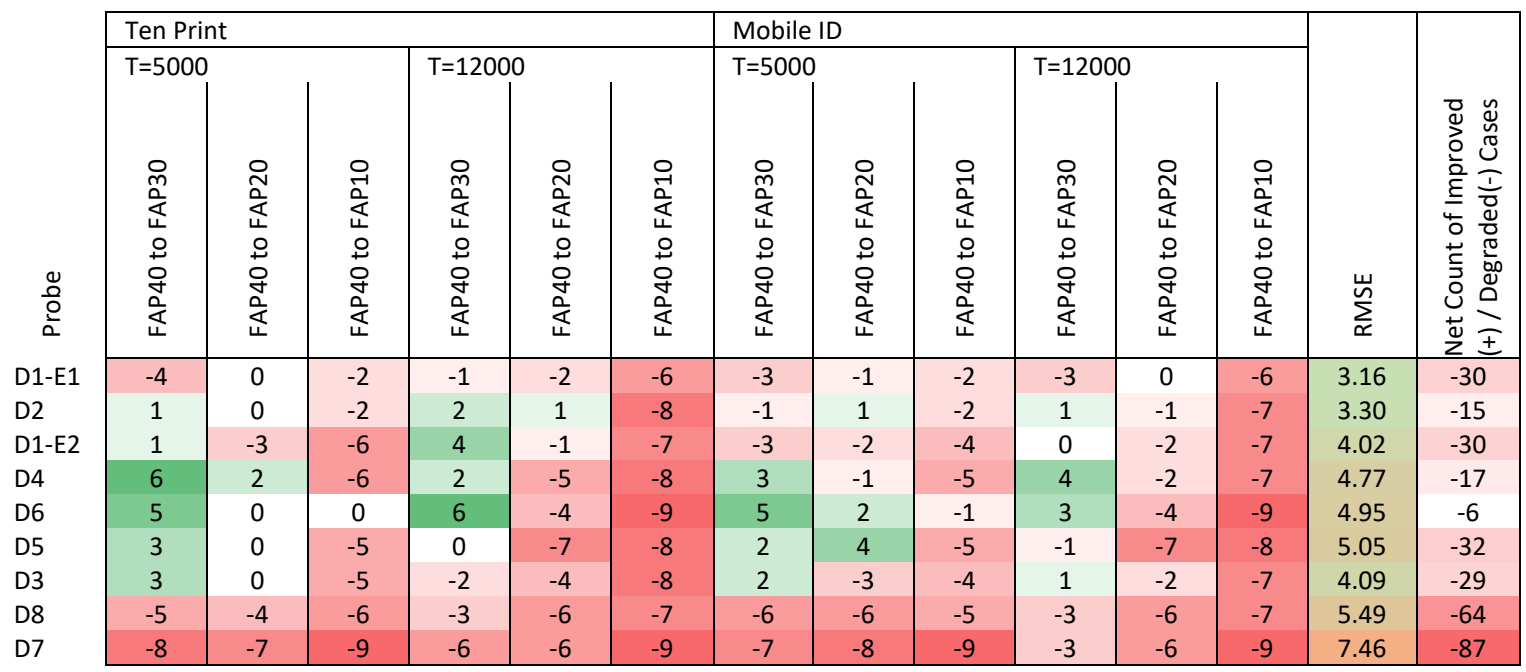

Table 23 - Measured Change in FNIR $(\Delta \%)$ at Level-set Threshold Resulting From Trimming Image From FAP40 Using a Contact-Collected Biometric Reference Database.

\begin{tabular}{|c|c|c|c|c|c|c|c|c|c|c|c|c|}
\hline & \multicolumn{6}{|c|}{ Ten-Print } & \multicolumn{6}{|c|}{ Mobile ID } \\
\hline & \multicolumn{3}{|c|}{ FNIR $(\Delta \%), T=5000$} & \multicolumn{3}{|c|}{ FNIR $(\Delta \%), T=12000$} & \multicolumn{3}{|c|}{ FNIR $(\Delta \%), T=5000$} & \multicolumn{3}{|c|}{ FNIR $(\Delta \%), T=12000$} \\
\hline & $\begin{array}{l}\text { FAP40 to } \\
\text { FAP30 }\end{array}$ & $\begin{array}{l}\text { FAP40 to } \\
\text { FAP20 }\end{array}$ & $\begin{array}{l}\text { FAP40 to } \\
\text { FAP10 }\end{array}$ & $\begin{array}{l}\text { FAP40 to } \\
\text { FAP30 }\end{array}$ & $\begin{array}{l}\text { FAP40 to } \\
\text { FAP20 }\end{array}$ & $\begin{array}{l}\text { FAP40 to } \\
\text { FAP10 }\end{array}$ & $\begin{array}{l}\text { FAP40 to } \\
\text { FAP30 }\end{array}$ & $\begin{array}{l}\text { FAP40 to } \\
\text { FAP20 }\end{array}$ & $\begin{array}{l}\text { FAP40 to } \\
\text { FAP10 }\end{array}$ & $\begin{array}{l}\text { FAP40 to } \\
\text { FAP30 }\end{array}$ & $\begin{array}{l}\text { FAP40 to } \\
\text { FAP20 }\end{array}$ & $\begin{array}{l}\text { FAP40 to } \\
\text { FAP10 }\end{array}$ \\
\hline D1-E2 4D1-E1 & 0 & -0.5 & 0 & 0 & 0 & 0 & 0 & -0.5 & 0 & 0 & 0 & 0 \\
\hline $\mathrm{D} 1-\mathrm{E} 1 \triangleleft \mathrm{D} 4$ & -0.5 & -0.5 & 0.5 & -1.6 & -1.1 & 2.1 & -0.5 & -0.5 & 0.5 & -1 & -0.5 & 2.6 \\
\hline D1-E1 <D1-E2 & 0 & 0 & 0 & 0 & 0 & 0 & 0 & 0 & 0 & 0 & 0 & 0 \\
\hline D2 $4 \mathrm{D} 1-\mathrm{E} 2$ & 0 & 0 & 0 & 0 & 0 & 0 & 0 & 0 & 0 & 0 & 0 & 0 \\
\hline D1-E1 $4 \mathrm{D} 2$ & 0 & 0 & 0 & 0 & 0 & 0.8 & 0 & 0 & 0 & 0 & 0 & 0.8 \\
\hline $\mathrm{D} 1-\mathrm{E} 2<\mathrm{D} 2$ & 0 & 0 & 0 & 0 & 0 & 0.8 & 0 & 0 & 0 & 0 & 0 & 0.8 \\
\hline $\mathrm{D} 1-\mathrm{E} 1 \triangleleft \mathrm{D} 6$ & -0.6 & -1.2 & -1.2 & -1.2 & 0 & 5.7 & -0.6 & -1.2 & -1.2 & -1.9 & 0 & 5.7 \\
\hline $\mathrm{D} 2 \triangleleft \mathrm{D} 4$ & 0 & 0.8 & 0.8 & 0 & 0 & 1.7 & -0.9 & 0.8 & 0 & -0.8 & 0 & 0 \\
\hline $\mathrm{D} 1-\mathrm{E} 2 \triangleleft \mathrm{D} 4$ & -0.5 & -0.5 & -0.5 & 1.1 & 0.5 & 3.7 & -1.1 & -1.1 & -1.6 & 0.6 & -0.5 & 2.7 \\
\hline D1-E1 $\triangleleft \mathrm{D} 3$ & -1 & -0.5 & 0 & 0 & 1 & 2.1 & -1 & -0.5 & 0 & 0 & 1 & 2.1 \\
\hline $\mathrm{D} 2<\mathrm{D} 1-\mathrm{E} 1$ & 0.8 & 0 & 0 & 0.8 & 0 & 0 & 0.8 & 0 & 0 & 0.8 & 0 & 0 \\
\hline $\mathrm{D} 2 \triangleleft \mathrm{D} 5$ & -0.8 & -1.7 & -0.8 & -1.7 & 1.6 & 6.7 & 0 & -1.7 & 0 & -0.9 & 0.8 & 5.8 \\
\hline $\mathrm{D} 1-\mathrm{E} 2 \triangleleft \mathrm{D} 6$ & 0 & 0 & -0.6 & -1.2 & -1.2 & 5.1 & 0.6 & 0.6 & 0 & -0.6 & -0.6 & 5.7 \\
\hline $\mathrm{D} 1-\mathrm{E} 1 \triangleleft \mathrm{D} 8$ & 2.1 & 1.1 & 1.1 & -1 & -1.6 & 3.7 & 2.1 & 1.1 & 1.6 & -1 & -1.6 & 4.2 \\
\hline $\mathrm{D} 2 \triangleleft \mathrm{D} 3$ & 0 & 0 & 0.8 & 0.8 & 2.5 & 2.5 & 0 & 0 & 0.8 & 0.8 & 2.5 & 2.5 \\
\hline $\mathrm{D} 2 \triangleleft \mathrm{D} 6$ & -1 & 0 & 0 & -2 & 1 & 9.2 & -1 & 0 & 0 & -1 & 2 & 9.2 \\
\hline $\mathrm{D} 1-\mathrm{E} 2 \triangleleft \mathrm{D} 3$ & 0.5 & 0.5 & 2.6 & 1 & 0.5 & 2.6 & 0.5 & 0.5 & 2.6 & 1.5 & 1 & 3.1 \\
\hline $\mathrm{D} 1-\mathrm{E} 1 \triangleleft \mathrm{D} 5$ & 0.5 & -1.6 & 3.1 & 1.5 & 0.5 & 8.3 & 0 & -1.6 & 2.6 & 1.6 & 0.6 & 7.3 \\
\hline $\mathrm{D} 1-\mathrm{E} 2 \triangleleft \mathrm{D} 8$ & 1.5 & 1.5 & 2.6 & 1.6 & 0 & 6.3 & 1.5 & 1.5 & 2.6 & 1.6 & 0 & 6.3 \\
\hline $\mathrm{D} 2 \triangleleft \mathrm{D} 8$ & 0.8 & -0.9 & 2.5 & 0 & 0.8 & 9.3 & 0.9 & 0 & 2.6 & 0 & 1.7 & 9.3 \\
\hline $\mathrm{D} 1-\mathrm{E} 2 \triangleleft \mathrm{D} 5$ & 0 & -0.5 & 3.1 & 2.1 & 2.6 & 10.9 & 1 & 1 & 3.6 & 4.2 & 4.2 & 12.5 \\
\hline $\mathrm{D} 1-\mathrm{E} 1 \triangleleft \mathrm{D} 7$ & 2.1 & 5.2 & 11.4 & -3.1 & 4.1 & 13.5 & 1 & 4.7 & 9.4 & -3.6 & 3.2 & 12 \\
\hline $\mathrm{D} 1-\mathrm{E} 2 \triangleleft \mathrm{D} 7$ & 1.6 & 6.3 & 12 & -1.6 & 4.1 & 15.6 & 0 & 6.3 & 12 & 2.1 & 7.8 & 17.7 \\
\hline $\mathrm{D} 2 \triangleleft \mathrm{D} 7$ & 1.6 & 2.5 & 10 & 5.1 & 9.3 & 26.1 & 1.6 & 4.2 & 8.4 & 4.2 & 10.9 & 26 \\
\hline
\end{tabular}




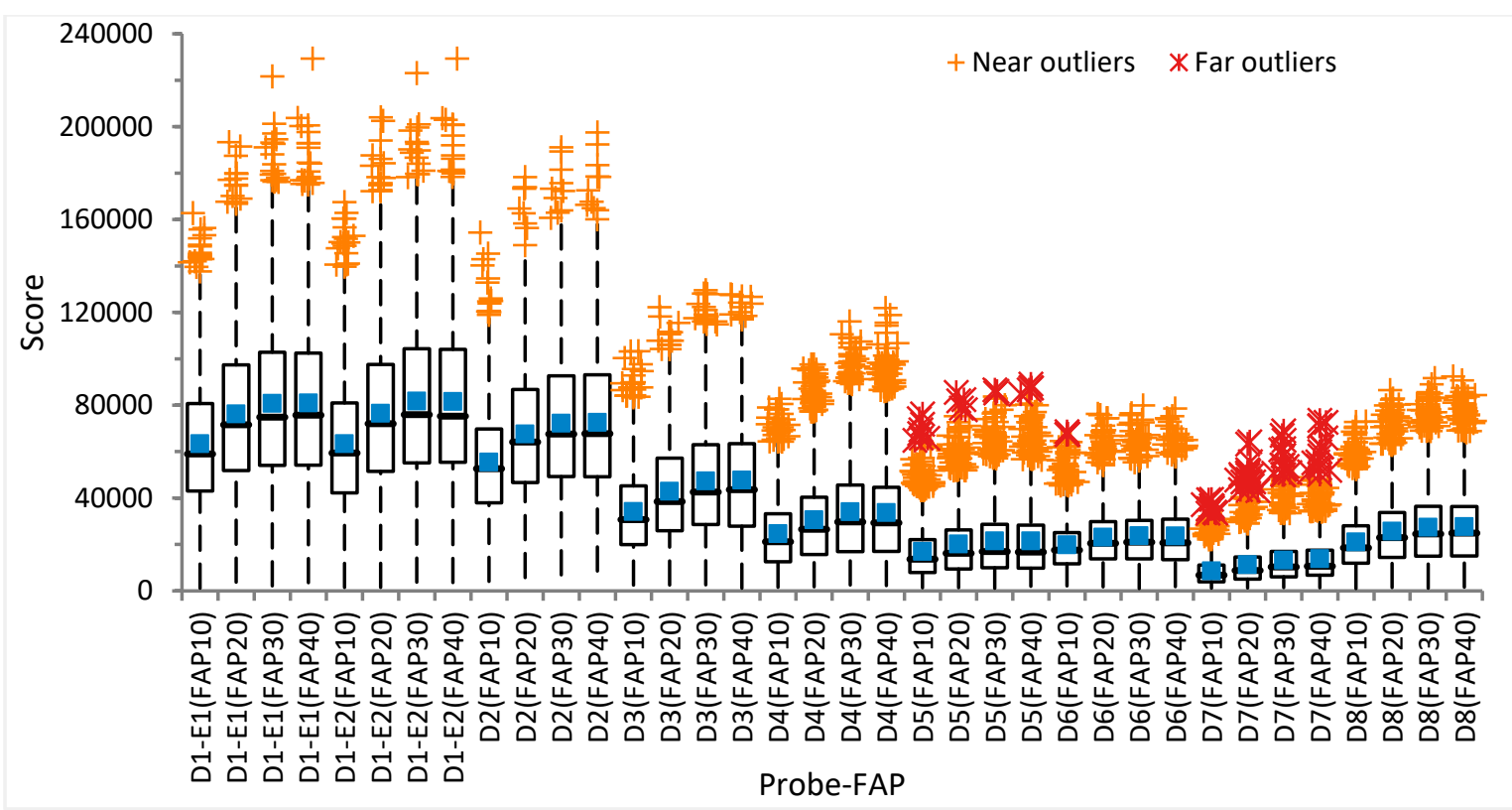

Figure 10 - Ten-Print Matcher Score Distribution by Probe FAP Size and Device (vs. Contact-Collected Biometric Reference Database).

Table 24 - Steel's Comparison of all Ten-Print Trials by Probe FAP Size and Device vs Control (D1-E1).

\begin{tabular}{|c|c|c|c|c|}
\hline Contrast & $\begin{array}{c}\text { Hodges- } \\
\text { Lehmann } \\
\text { location shift }\end{array}$ & Simultaneous $95 \% \mathrm{Cl}$ & 0 & $\mathrm{p}$-value \\
\hline D1-E2(FAP30) - D1-E1(FAP40) & 824.0 & -3511.0 to 5158.0 & TI & 1.0000 \\
\hline D1-E2(FAP40) - D1-E1(FAP40) & 658.0 & -3652.0 to 4962.0 & il & 1.0000 \\
\hline D1-E1(FAP30) - D1-E1(FAP40) & -52.0 & -4376.0 to 4267.0 & II & 1.0000 \\
\hline D1-E2(FAP20) - D1-E1(FAP40) & -3824.0 & -8019.0 to 349.0 & $\mathbf{\square}$ & 0.0978 \\
\hline D1-E1(FAP20) - D1-E1(FAP40) & -3989.0 & -8161.0 to 146.0 & [ & 0.0669 \\
\hline D2(FAP40) - D1-E1(FAP40) & -7215.5 & -11583.0 to -2877.0 & [ & $<0.0001$ \\
\hline D2 (FAP30) - D1-E1(FAP40) & -7427.0 & -11811.0 to -3105.0 & [ & $<0.0001$ \\
\hline D2(FAP20) - D1-E1(FAP40) & -11483.0 & -15699.0 to -7333.0 & [ & $<0.0001$ \\
\hline D1-E1(FAP10) - D1-E1(FAP40) & -15761.0 & -19658.0 to -11909.0 & [ & $<0.0001$ \\
\hline D1-E2(FAP10) - D1-E1(FAP40) & -15937.0 & -19869.0 to -12066.0 & [ & $<0.0001$ \\
\hline D2(FAP10) - D1-E1(FAP40) & -22861.0 & -26886.0 to -18927.0 & [ & $<0.0001$ \\
\hline D3(FAP40) - D1-E1(FAP40) & -31075.0 & -34468.0 to -27717.0 & [ & $<0.0001$ \\
\hline D3(FAP30) - D1-E1(FAP40) & -31317.0 & -34695.0 to -27975.0 & [ & $<0.0001$ \\
\hline D3(FAP20) - D1-E1(FAP40) & -35313.0 & -38616.0 to -32054.0 & [ & $<0.0001$ \\
\hline D3(FAP10) - D1-E1(FAP40) & -43182.0 & -46393.0 to -40073.0 & [ & $<0.0001$ \\
\hline D4(FAP30) - D1-E1(FAP40) & -44036.0 & -47283.0 to -40825.0 & [ & $<0.0001$ \\
\hline D4(FAP40) - D1-E1(FAP40) & -44408.0 & -47659.0 to -41209.0 & [ & $<0.0001$ \\
\hline D4(FAP20) - D1-E1(FAP40) & -47006.0 & -50227.0 to -43863.0 & [ & $<0.0001$ \\
\hline D8(FAP40) - D1-E1(FAP40) & -49495.0 & -52648.0 to -46410.0 & [ & $<0.0001$ \\
\hline D8(FAP30) - D1-E1(FAP40) & -49671.0 & -52839.0 to -46588.0 & [ & $<0.0001$ \\
\hline D8(FAP20) - D1-E1(FAP40) & -51151.0 & -54325.0 to -48081.0 & [ & $<0.0001$ \\
\hline D4(FAP10) - D1-E1(FAP40) & -52199.0 & -55382.0 to -49129.0 & [ & $<0.0001$ \\
\hline D6(FAP30) - D1-E1(FAP40) & -52776.0 & -56050.0 to -49649.0 & $\bar{\square}$ & $<0.0001$ \\
\hline D6(FAP40) - D1-E1(FAP40) & -52895.0 & -56153.0 to -49763.0 & घ & $<0.0001$ \\
\hline D6(FAP20) - D1-E1(FAP40) & -53359.0 & -56625.0 to -50201.0 & [ & $<0.0001$ \\
\hline D5(FAP30) - D1-E1(FAP40) & -55274.0 & -58494.0 to -52201.0 & [ & $<0.0001$ \\
\hline D5(FAP40) - D1-E1(FAP40) & -55331.0 & -58561.0 to -52264.0 & $\mathbf{\square}$ & $<0.0001$ \\
\hline D8(FAP10) - D1-E1(FAP40) & -55349.0 & -58527.0 to -52287.0 & [ & $<0.0001$ \\
\hline D6(FAP10) - D1-E1(FAP40) & -56445.0 & -59756.0 to -53270.0 & [ & $<0.0001$ \\
\hline D5(FAP20) - D1-E1(FAP40) & -56453.0 & -59663.0 to -53384.0 & [ & $<0.0001$ \\
\hline D5(FAP10) - D1-E1(FAP40) & -59375.0 & -62584.0 to -56282.0 & I & $<0.0001$ \\
\hline D7(FAP40) - D1-E1(FAP40) & -62344.0 & -65755.0 to -59072.0 & [ & $<0.0001$ \\
\hline D7(FAP30) - D1-E1(FAP40) & -62873.0 & -66312.0 to -59562.0 & $\mathbf{\square}$ & $<0.0001$ \\
\hline D7(FAP20) - D1-E1(FAP40) & -64739.0 & -68219.0 to -61377.0 & घ & $<0.0001$ \\
\hline D7(FAP10) - D1-E1(FAP40) & -67235.0 & -70778.0 to -63796.0 & 口 & $<0.0001$ \\
\hline
\end{tabular}




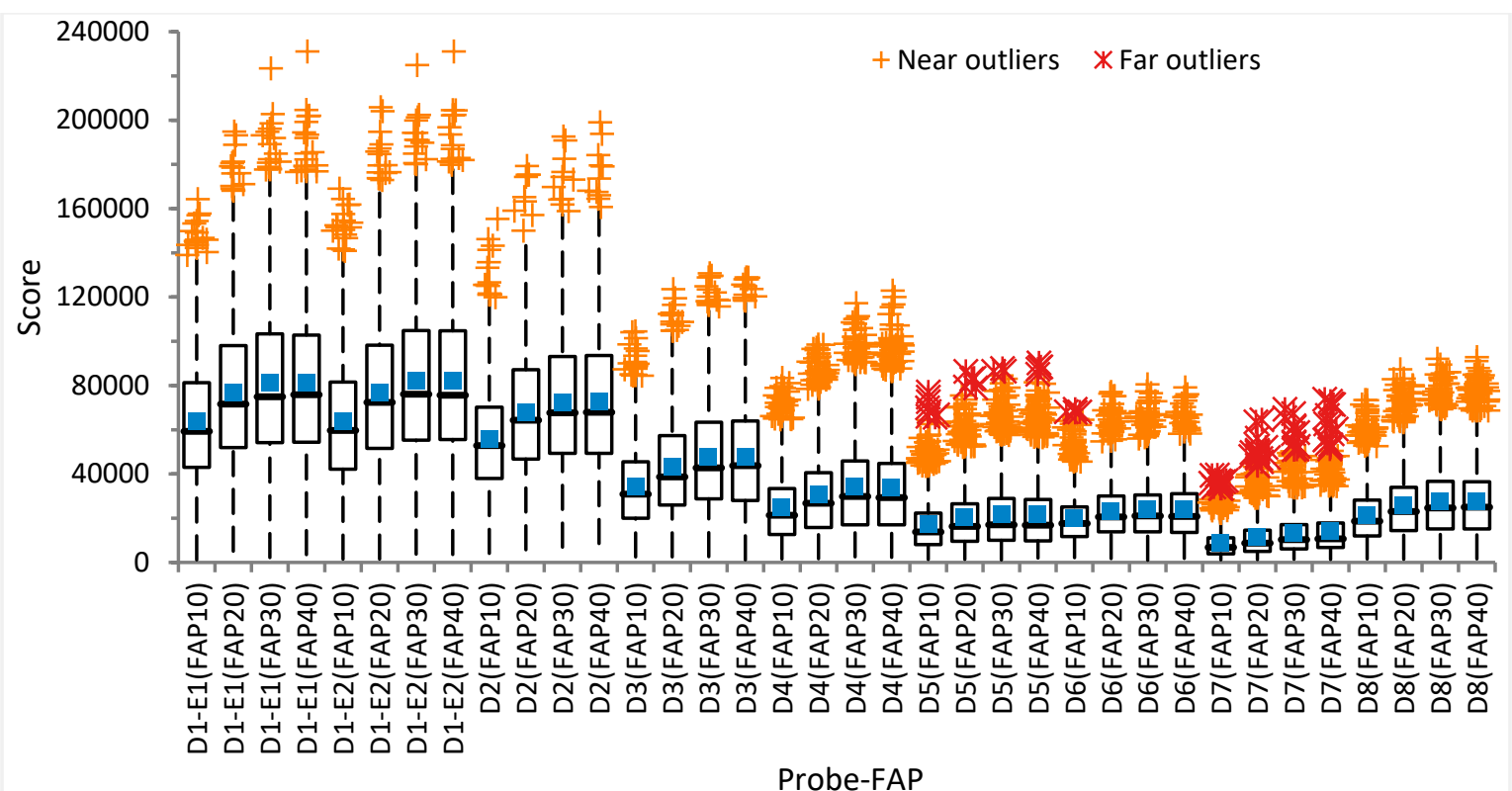

Figure 11 - Mobile ID Matcher Score Distribution by Probe FAP Size and Device Using a Contact-Collected Biometric Reference Database.

Table 25 - Steel's Comparison of all Mobile ID Trials by Probe FAP Size and Device vs Control (D1-E1).

\begin{tabular}{|c|c|c|c|c|}
\hline Contrast & $\begin{array}{c}\text { Hodges- } \\
\text { Lehmann } \\
\text { location shift }\end{array}$ & Simultaneous $95 \% \mathrm{Cl}$ & 0 & $\mathrm{p}$-value \\
\hline D1-E2(FAP30) - D1-E1(FAP40) & 824.0 & -3536.0 to 5191.0 & II & 1.0000 \\
\hline D1-E2(FAP40) - D1-E1(FAP40) & 657.0 & -3685.0 to 4988.0 & il & 1.0000 \\
\hline D1-E1(FAP30) - D1-E1(FAP40) & -56.0 & -4411.0 to 4285.0 & II & 1.0000 \\
\hline D1-E2(FAP2O) - D1-E1(FAP40) & -3828.0 & -8050.0 to 373.0 & [ & 0.1012 \\
\hline D1-E1(FAP20) - D1-E1(FAP40) & -3943.0 & -8146.0 to 216.0 & [ & 0.0771 \\
\hline D2(FAP40) - D1-E1(FAP40) & -7248.0 & -11637.0 to -2871.0 & [ & $<0.0001$ \\
\hline D2(FAP30) - D1-E1(FAP40) & -7448.5 & -11870.0 to -3097.0 & $\mathbf{\square}$ & $<0.0001$ \\
\hline D2 (FAP20) - D1-E1(FAP40) & -11499.0 & -15744.0 to -7326.0 & $\mathbf{E}$ & $<0.0001$ \\
\hline D1-E1(FAP10) - D1-E1(FAP40) & -15776.0 & -19708.0 to -11899.0 & $\mathbf{\square}$ & $<0.0001$ \\
\hline D1-E2(FAP10) - D1-E1(FAP40) & -15951.0 & -19916.0 to -12055.0 & E & $<0.0001$ \\
\hline D2(FAP10) - D1-E1(FAP40) & -22885.0 & -26942.0 to -18920.0 & $\mathbf{D}$ & $<0.0001$ \\
\hline D3(FAP40) - D1-E1(FAP40) & -31125.0 & -34537.0 to -27741.0 & $\mathbf{\square}$ & $<0.0001$ \\
\hline D3(FAP30) - D1-E1(FAP40) & -31367.0 & -34765.0 to -28004.0 & [ & $<0.0001$ \\
\hline D3(FAP20) - D1-E1(FAP40) & -35400.0 & -38722.0 to -32118.0 & [ & $<0.0001$ \\
\hline D3(FAP10) - D1-E1(FAP40) & -43256.0 & -46497.0 to -40123.0 & 匹 & $<0.0001$ \\
\hline D4(FAP30) - D1-E1(FAP40) & -44099.0 & -47373.0 to -40865.0 & [ & $<0.0001$ \\
\hline D4(FAP40) - D1-E1(FAP40) & -44519.0 & -47797.0 to -41305.0 & $\overline{\mathbf{z}}$ & $<0.0001$ \\
\hline D4(FAP20) - D1-E1(FAP40) & -47052.0 & -50298.0 to -43881.0 & [ & $<0.0001$ \\
\hline D8(FAP40) - D1-E1(FAP40) & -49626.0 & -52812.0 to -46521.0 & [ & $<0.0001$ \\
\hline D8(FAP30) - D1-E1(FAP40) & -49726.0 & -52921.0 to -46618.0 & E & $<0.0001$ \\
\hline D8(FAP20) - D1-E1(FAP40) & -51291.0 & -54496.0 to -48199.0 & [ & $<0.0001$ \\
\hline D4(FAP10) - D1-E1(FAP40) & -52233.0 & -55451.0 to -49135.0 & [ & $<0.0001$ \\
\hline D6(FAP30) - D1-E1(FAP40) & -52904.0 & -56201.0 to -49746.0 & - & $<0.0001$ \\
\hline D6(FAP40) - D1-E1(FAP40) & -53006.0 & -56291.0 to -49850.0 & [ & $<0.0001$ \\
\hline D6(FAP20) - D1-E1(FAP40) & -53490.0 & -56792.0 to -50308.0 & [ & $<0.0001$ \\
\hline D5(FAP30) - D1-E1(FAP40) & -55400.0 & -58645.0 to -52301.0 & [ & $<0.0001$ \\
\hline D5(FAP40) - D1-E1(FAP40) & -55416.0 & -58670.0 to -52319.0 & [ & $<0.0001$ \\
\hline D8(FAP10) - D1-E1(FAP40) & -55467.0 & -58673.0 to -52370.0 & [ & $<0.0001$ \\
\hline D5(FAP2O) - D1-E1(FAP40) & -56541.0 & -59780.0 to -53440.0 & [ & $<0.0001$ \\
\hline D6(FAP10) - D1-E1(FAP40) & -56575.0 & -59920.0 to -53368.0 & [ & $<0.0001$ \\
\hline D5(FAP10) - D1-E1(FAP40) & -59460.0 & -62696.0 to -56335.0 & $\mathbf{E}$ & $<0.0001$ \\
\hline D7(FAP40) - D1-E1(FAP40) & -62428.0 & -65899.0 to -59123.0 & [ & $<0.0001$ \\
\hline D7(FAP30) - D1-E1(FAP40) & -63066.0 & -66533.0 to -59720.0 & $\overline{\mathbf{n}}$ & $<0.0001$ \\
\hline D7(FAP20) - D1-E1(FAP40) & -64937.0 & -68451.0 to -61545.0 & $\mathbf{E}$ & $<0.0001$ \\
\hline D7(FAP10) - D1-E1(FAP40) & -67421.0 & -71010.0 to -63951.0 & & $<0.0001$ \\
\hline
\end{tabular}




\subsection{Objective 5: Comparison of Matcher Throughput (Ten-Print vs. Mobile ID matcher) with respect to reduced image size and reduced finger count}

\begin{tabular}{ll}
\hline Biometric Reference Database Composition: & Two encounters for D1, and D2, 8 fingers, full size (FAP40) \\
Probe Set Composition: & All 8 devices, two encounters for D1, all finger combinations, all FAPs \\
Matching Threshold: & N/A \\
Special Notes: & Also includes contact-to-contact cases for comparison \\
Number of test trials in this Objective: & $\mathrm{P}(9,2) \times 2=144$ (72 on Mobile ID, and 72 on Ten-Print) \\
\hline
\end{tabular}

While the other objectives in this study were primarily focused on measuring the accuracy of the matchers when operating on contactless images of reduced size or reduced finger count, another important operational concern is the throughput of the matcher under similar constraints. A matcher with perfect accuracy may be of limited utility if the amount of time it consumes in conducting searches increases substantially.

The goal of this objective is to see if a Mobile ID matcher performs better in terms of transactional throughput when compared to a Ten-Print matcher using the same experimental data with no changes to the data to favor either matcher.

Both matcher configurations utilized a biometric reference database consisting of only contact collected images. Probes consisted of all devices, all image sizes and all finger combinations.

\subsubsection{Findings for Objective 5}

Examination of cases with reduced finger counts (from 8 finger to 6, 4 and 2 fingers), the median matcher service time showed a linear decrease with reduced fingers on both the Ten-Print matcher (Table 26, Figure 12a) and the Mobile ID matcher (Table 27, Figure 12b). The difference between each trial was statistically significant at $(\mathrm{p}<0.05)$ using Steel's multiple comparison test [11]. The treatments were well separated, and the smallest difference in median time was $8341 \mathrm{~ms}$ (case $8 \mathrm{~F}$ vs $6 \mathrm{~F})$.

Examination of cases with reduced image size (from FAP40 to FAP30, FAP20 and FAP10), the medians of service times did not show a clear trend. The case that had the lowest service time for the Ten-Print matcher was that of FAP20 (Table 28) with a median service time of $22190 \mathrm{~ms}$. For the Mobile ID matcher, the case with the lowest service time was that of FAP30 (Table 29).

Examination of matcher service time distribution with reduced image sizes (Figure $12 \mathrm{c}$ and d) shows that while the populations were nearly the same, service times for the larger image size trials had many more outliers. Despite having service time distributions that were very close to each other, the difference between the service time trials was statistically significant at $(\mathrm{p}<0.05)$ for all trials (Table 28, Table 29).

Extending this analysis further to the device level for the Ten-Print matcher (Figure 13 and Table 30) and Mobile ID matcher (Figure 14 and Table 31) again shows tight clustering of all device trials without a clear generalizable pattern. Pairwise examination of the trials for the Ten-Print matcher (Table 32) and Mobile ID matcher (Table 33) using Steel's comparison versus the control case (D1-E1 at FAP40) did show that most cases differed from the control and this difference was statistically significant ( $\mathrm{p}<0.05)$. Five cases, namely D1-E1(FAP20 and FAP30), D1-E2(FAP20,FAP30 and FAP40), had timing distributions that were indistinguishable from each other. 


\subsubsection{Data for Objective 5}

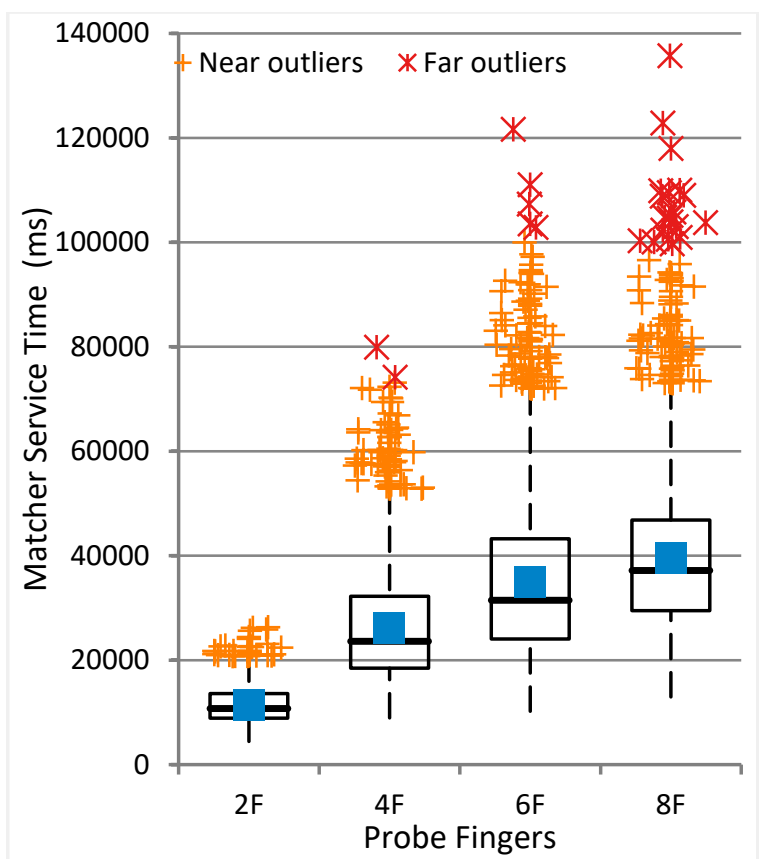

a. Ten-Print Matcher Throughput by Probe Finger Count.

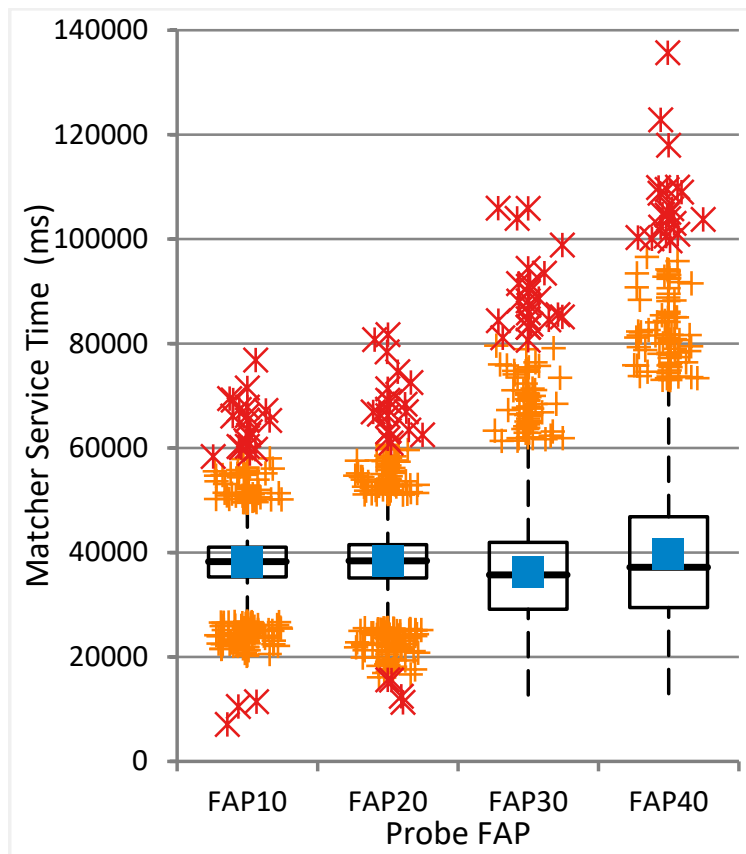

c. Ten-Print Matcher Throughput by Probe FAP Size.

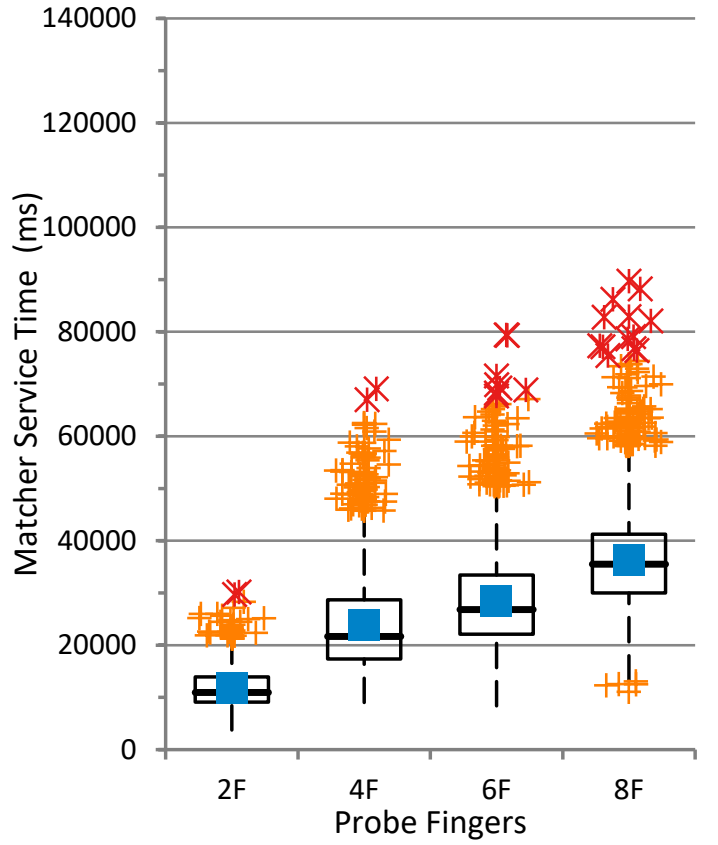

b. Mobile ID Matcher Throughput by Probe Finger Count.

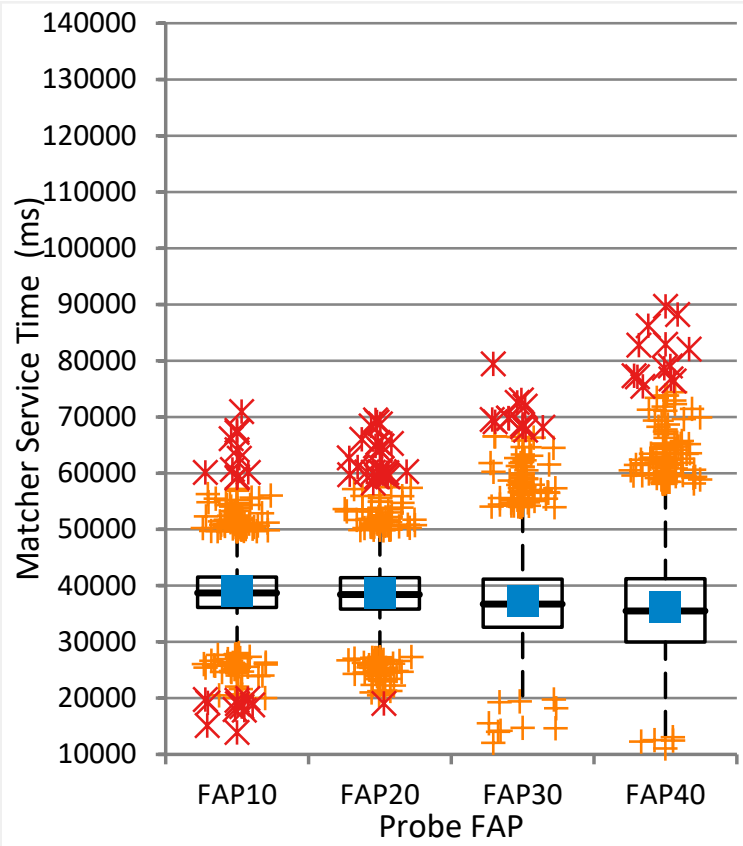

D. Mobile Id Matcher Throughput by Probe Fap Size.

Figure 12- Matcher Throughput With Respect to the Number of Fingers, and Finger Image Size Provided as the Search Probe Using a Contact-Collected Biometric Reference Database. 
Table 26 - Steel's Multiple Comparison Rank Sum Test of Ten-Print Matcher Cases, Grouped by Probe Finger Count Versus Control Case (8F).

\begin{tabular}{c|c|c|c|c|c|c|c}
$\begin{array}{c}\text { Matcher Service Time (ms) } \\
\text { by Probe Fingers }\end{array}$ & Mean & Median & 1st Quartile & 3rd Quartile & IQR & Hodges-Lehmann location shift (vs 8F) & p-value \\
\hline $2 \mathrm{~F}$ & 11464.1 & 10738.5 & 8895.3 & 13614.2 & 4718.9 & -5011.0 \\
\hline $4 \mathrm{~F}$ & 26081.4 & 23609.0 & 18478.8 & 32215.8 & 13737.0 & $<0.0001$ \\
\hline $6 \mathrm{~F}$ & 34881.4 & 31467.0 & 24058.5 & 43221.0 & 19162.5 & -12542.0 \\
\hline $8 \mathrm{~F}$ & 39619.2 & 37168.0 & 29463.9 & 46837.3 & 17373.3 & $<0.0001$ \\
\hline NA &
\end{tabular}

Table 27 - Steel's Multiple Comparison Rank Sum Test of Mobile ID Matcher Cases, Grouped by Probe Finger Count Versus Control Case (8F).

\begin{tabular}{c|c|c|c|c|c|c}
$\begin{array}{c}\text { Matcher Service Time (ms) } \\
\text { by Probe Fingers }\end{array}$ & Mean & Median & 1st Quartile & 3rd Quartile & IQR & Hodges-Lehmann location shift (vs 8F) \\
\hline $2 \mathrm{~F}$ & 11705.4 & 10940.0 & 9088.2 & 13927.7 & 4839.5 & p-value \\
\hline $4 \mathrm{~F}$ & 23767.2 & 21665.0 & 17345.0 & 28676.3 & 11331.3 & -8084.0 \\
\hline $6 \mathrm{~F}$ & 28478.5 & 26814.0 & 22106.2 & 33424.3 & 11318.2 & -12839.0 \\
\hline $8 \mathrm{~F}$ & 36273.7 & 35503.0 & 29981.2 & 41234.6 & 11253.4 & $<0.0001$ \\
\hline
\end{tabular}

Table 28 - Steel's Multiple Comparison Rank Sum Test of Ten-Print Matcher Cases, Grouped by Probe FAP Size Versus Control Case (FAP40).

\begin{tabular}{|c|c|c|c|c|c|c|c|}
\hline $\begin{array}{c}\text { Matcher Service Time (ms) } \\
\text { by Probe FAP }\end{array}$ & Mean & Median & 1st Quartile & 3rd Quartile & $\mathrm{IQR}$ & Hodges-Lehmann location shift (vs FAP40) & $\mathrm{p}$-value \\
\hline FAP10 & 38139.7 & 38255.0 & 35320.3 & 41002.7 & 5682.3 & 853.0 & 0.0009 \\
\hline FAP20 & 38390.6 & 38402.0 & 35137.0 & 41512.3 & 6375.3 & 666.0 & 0.0113 \\
\hline FAP30 & 36274.4 & 35714.0 & 29141.8 & 41951.3 & 12809.5 & -2231.0 & $<0.0001$ \\
\hline FAP40 & 39619.2 & 37168.0 & 29463.9 & 46837.3 & 17373.3 & NA & NA \\
\hline
\end{tabular}

Table 29 - Steel's Multiple Comparison Rank Sum Test of Mobile ID Matcher Cases, Grouped by Probe FAP Size Versus Control Case (FAP40).

\begin{tabular}{c|c|c|c|c|c|c}
$\begin{array}{c}\text { Matcher Service Time (ms) } \\
\text { by Probe FAP }\end{array}$ & Mean & Median & 1st Quartile & 3rd Quartile & IQR & Hodges-Lehmann location shift (vs FAP40) \\
\hline FAP10 & 39053.3 & 38708.0 & 36144.5 & 41528.7 & 5384.2 & p-value \\
\hline FAP20 & 38752.5 & 38424.0 & 35838.8 & 41421.1 & 5582.3 & 3395.0 \\
\hline FAP30 & 37316.7 & 36726.5 & 32600.8 & 41150.0 & 8549.2 & 3067.0 \\
\hline FAP40 & 36273.7 & 35503.0 & 29981.2 & 41234.6 & 11253.4 & $<0001$ \\
\hline
\end{tabular}




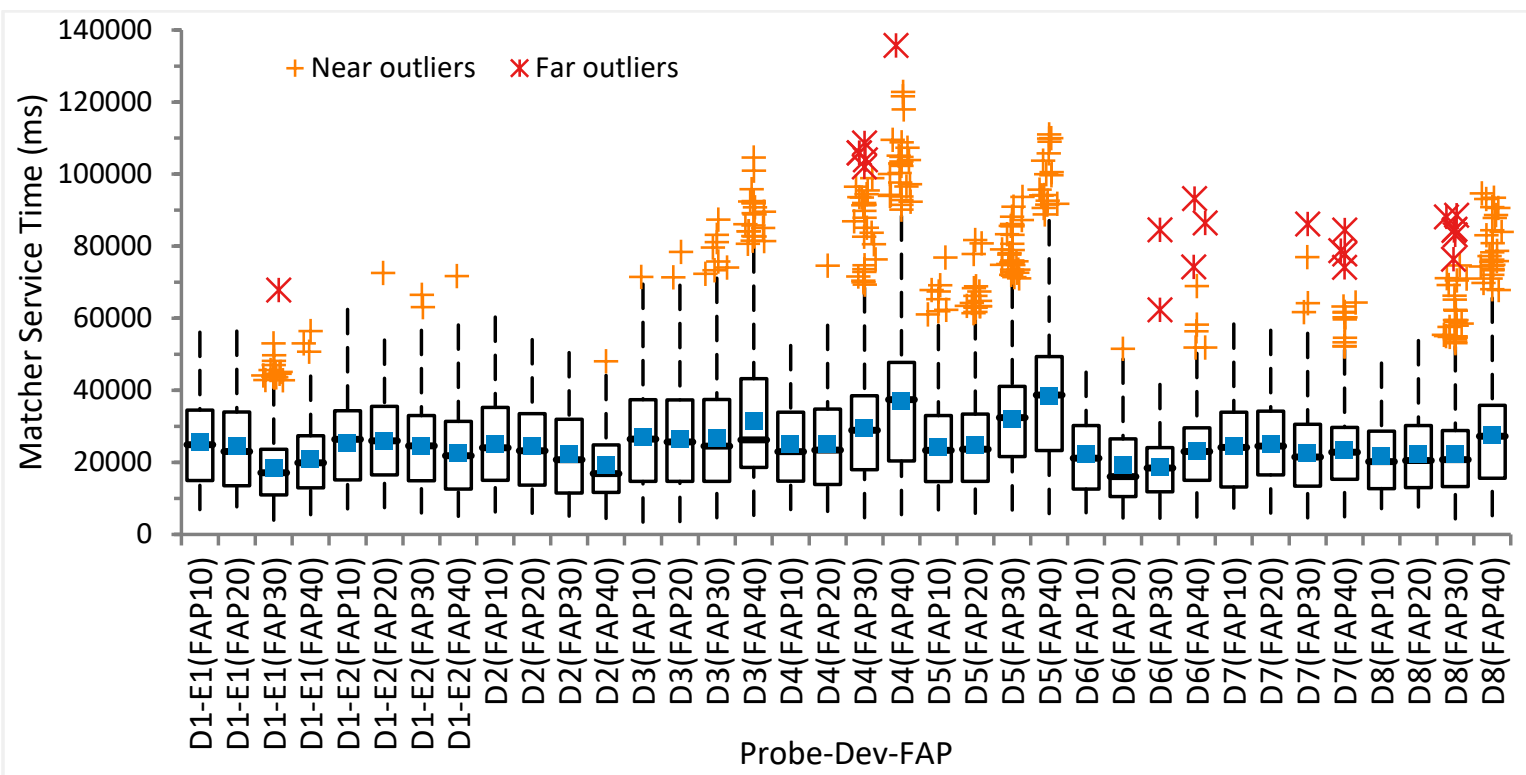

Figure 13 - Ten-Print Throughput Distribution by Device and Probe FAP Size Using a Contact-Collected Biometric Reference Database.

Table 30 - Ten-Print Matcher Descriptive Throughput Data by Device and Probe FAP Size Using a ContactCollected Biometric Reference Database.

\begin{tabular}{|c|c|c|c|c|c|}
\hline $\begin{array}{c}\text { Matcher Service Time (ms) } \\
\text { by Probe-Dev-FAP }\end{array}$ & Mean & Median & 1st Quartile & 3rd Quartile & IQR \\
\hline D6(FAP20) & 19170.5 & 16052.0 & 10495.3 & 26501.3 & 16006.0 \\
\hline D2(FAP40) & 19279.2 & 16887.0 & 11680.0 & 24824.6 & 13144.6 \\
\hline D1-E1(FAP30) & 18461.5 & 17126.0 & 10990.2 & 23663.5 & 12673.3 \\
\hline D6(FAP30) & 18731.8 & 18435.0 & 11861.3 & 24121.7 & 12260.3 \\
\hline D1-E1(FAP40) & 20891.4 & 19888.0 & 12921.3 & 27391.0 & 14469.7 \\
\hline D8(FAP10) & 21743.2 & 20212.5 & 12730.8 & 28686.0 & 15955.2 \\
\hline D8(FAP20) & 22226.8 & 20515.0 & 13011.0 & 30249.7 & 17238.7 \\
\hline D2(FAP30) & 22200.7 & 20742.0 & 11474.8 & 31944.8 & 20469.9 \\
\hline D8(FAP30) & 22239.0 & 20764.5 & 13303.3 & 28835.9 & 15532.7 \\
\hline D6(FAP10) & 22263.0 & 21162.5 & 12600.4 & 30228.8 & 17628.3 \\
\hline D7(FAP30) & 22441.9 & 21492.0 & 13399.8 & 30583.5 & 17183.7 \\
\hline D1-E2(FAP40) & 22634.0 & 21853.0 & 12567.3 & 31368.7 & 18801.3 \\
\hline D7(FAP40) & 23271.2 & 22822.0 & 15310.0 & 29703.3 & 14393.3 \\
\hline D4(FAP10) & 25047.1 & 22982.0 & 14792.7 & 33930.7 & 19138.0 \\
\hline D1-E1(FAP20) & 24523.8 & 23021.0 & 13507.7 & 33961.7 & 20454.0 \\
\hline D6(FAP40) & 23145.7 & 23023.0 & 15033.2 & 29621.4 & 14588.3 \\
\hline D2(FAP20) & 24454.6 & 23224.0 & 13664.3 & 33529.3 & 19865.0 \\
\hline D5(FAP10) & 24322.3 & 23344.0 & 14663.7 & 32982.3 & 18318.7 \\
\hline D4(FAP20) & 25025.7 & 23412.0 & 13893.2 & 34795.8 & 20902.7 \\
\hline D5(FAP20) & 24905.8 & 23651.0 & 14739.7 & 33387.7 & 18648.0 \\
\hline D2(FAP10) & 25050.5 & 24059.5 & 15013.9 & 35246.9 & 20233.0 \\
\hline D7(FAP10) & 24618.3 & 24154.5 & 13192.8 & 33920.1 & 20727.3 \\
\hline D3(FAP30) & 26837.8 & 24513.0 & 14747.9 & 37473.3 & 22725.4 \\
\hline D7(FAP20) & 25185.4 & 24523.0 & 16539.9 & 34224.8 & 17684.8 \\
\hline D1-E2(FAP30) & 24351.9 & 24587.5 & 14912.6 & 32979.4 & 18066.8 \\
\hline D1-E1(FAP10) & 25513.3 & 24909.0 & 14943.7 & 34526.7 & 19583.0 \\
\hline D3(FAP20) & 26462.8 & 25680.0 & 14734.5 & 37360.7 & 22626.2 \\
\hline D1-E2(FAP20) & 25894.8 & 25988.0 & 16541.0 & 35530.3 & 18989.3 \\
\hline D3(FAP40) & 31483.7 & 26249.0 & 18586.8 & 43238.8 & 24652.1 \\
\hline D1-E2(FAP10) & 25360.5 & 26400.0 & 15134.8 & 34317.8 & 19183.0 \\
\hline D3(FAP10) & 27065.8 & 26463.0 & 14714.7 & 37402.7 & 22688.0 \\
\hline D8(FAP40) & 27500.3 & 27260.0 & 15623.3 & 35813.3 & 20190.0 \\
\hline D4(FAP30) & 29631.6 & 28910.5 & 17969.3 & 38494.0 & 20524.8 \\
\hline D5(FAP30) & 32076.9 & 32425.5 & 21603.0 & 41094.3 & 19491.3 \\
\hline D4(FAP40) & 36901.5 & 37416.5 & 20373.2 & 47769.2 & 27396.0 \\
\hline D5(FAP40) & 38271.8 & 38697.0 & 23303.0 & 49379.7 & 26076.7 \\
\hline
\end{tabular}




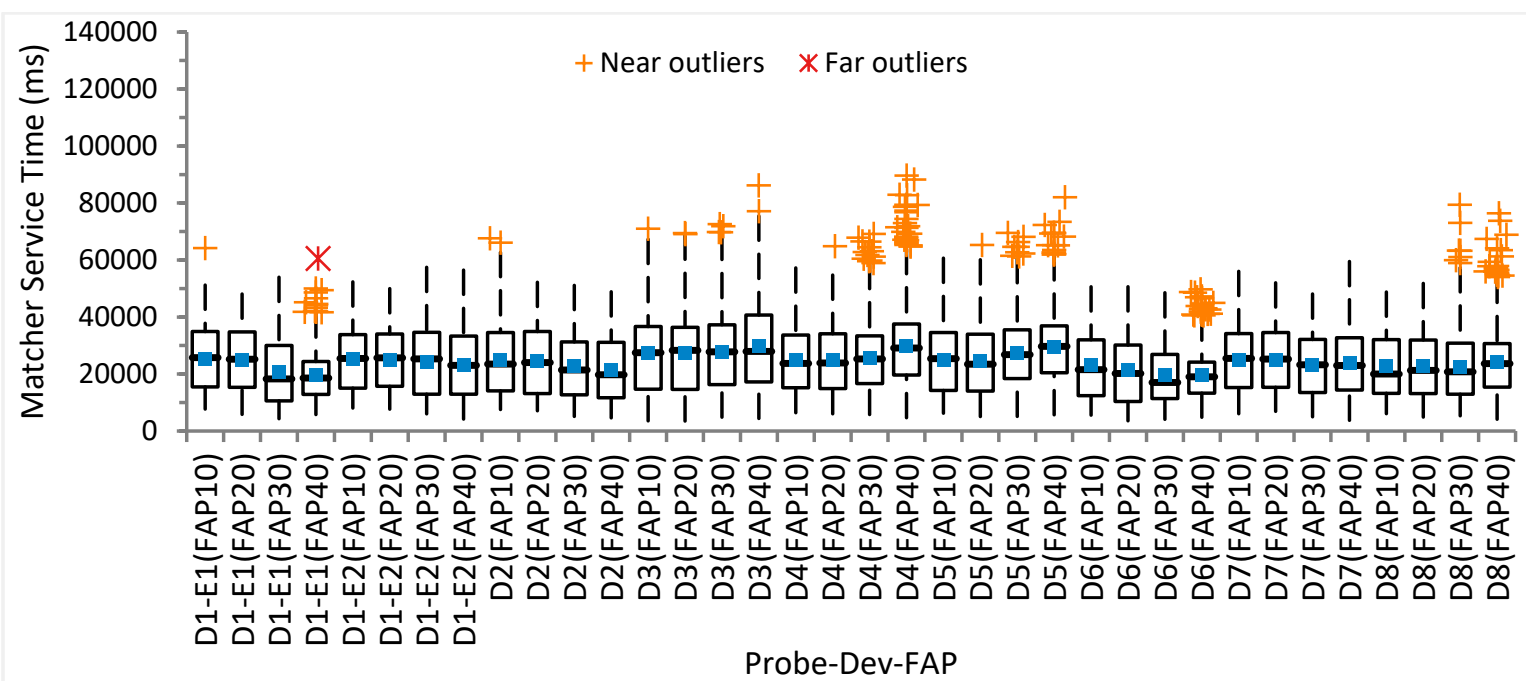

Figure 14 - Mobile ID Throughput Distribution by Device and Probe FAP Size Using a Contact-Collected Biometric Reference Database.

Table 31 - Mobile ID Matcher Descriptive Throughput Data by Device and Probe FAP Size Using a ContactCollected Biometric Reference Database.

\begin{tabular}{|c|c|c|c|c|c|}
\hline $\begin{array}{r}\text { Matcher Service Time (ms) } \\
\text { by Probe-Dev-FAP }\end{array}$ & Mean & Median & 1st Quartile & 3rd Quartile & IQR \\
\hline D6(FAP30) & 19516.9 & 17032.0 & 11352.3 & 26898.0 & 15545.7 \\
\hline D1-E1(FAP30) & 20765.3 & 18286.0 & 10567.0 & 30022.3 & 19455.3 \\
\hline D1-E1(FAP40) & 19688.6 & 18599.0 & 12846.3 & 24375.0 & 11528.7 \\
\hline D6(FAP40) & 19654.8 & 19027.5 & 13296.3 & 24201.0 & 10904.7 \\
\hline D2(FAP40) & 21591.4 & 19748.5 & 11671.2 & 31103.0 & 19431.8 \\
\hline D8(FAP10) & 22928.8 & 20005.0 & 13248.3 & 32093.0 & 18844.7 \\
\hline D6(FAP20) & 21359.0 & 20193.0 & 10355.0 & 30159.3 & 19804.3 \\
\hline D8(FAP30) & 22417.8 & 20780.5 & 12913.8 & 30817.4 & 17903.6 \\
\hline D8(FAP20) & 22970.7 & 21273.0 & 13133.0 & 31962.7 & 18829.7 \\
\hline D2(FAP30) & 22739.3 & 21418.0 & 12722.7 & 31261.8 & 18539.1 \\
\hline D6(FAP10) & 23064.9 & 21537.0 & 12403.3 & 32024.0 & 19620.7 \\
\hline D7(FAP40) & 23680.0 & 22997.0 & 14390.0 & 32759.3 & 18369.3 \\
\hline D1-E2(FAP40) & 23363.1 & 23039.0 & 12912.7 & 33292.3 & 20379.7 \\
\hline D7(FAP30) & 23084.6 & 23248.0 & 13527.3 & 32111.7 & 18584.4 \\
\hline D5(FAP20) & 24548.4 & 23452.5 & 14053.3 & 33944.9 & 19891.6 \\
\hline D2(FAP10) & 24940.9 & 23492.5 & 14070.8 & 34587.9 & 20517.2 \\
\hline D8(FAP40) & 24128.7 & 23653.0 & 15441.0 & 30715.3 & 15274.3 \\
\hline D4(FAP10) & 24934.6 & 23718.0 & 15188.7 & 33662.3 & 18473.7 \\
\hline D4(FAP20) & 24928.4 & 23879.0 & 14865.9 & 34133.3 & 19267.3 \\
\hline D2(FAP20) & 24420.2 & 24022.0 & 13171.8 & 34896.0 & 21724.3 \\
\hline D1-E1(FAP20) & 24821.5 & 25196.0 & 15315.3 & 34777.3 & 19461.9 \\
\hline D7(FAP20) & 24766.5 & 25249.0 & 15350.5 & 34531.5 & 19181.0 \\
\hline D4(FAP30) & 25766.7 & 25292.0 & 16621.5 & 33406.7 & 16785.2 \\
\hline D1-E2(FAP30) & 24351.2 & 25316.5 & 12941.6 & 34610.5 & 21668.9 \\
\hline D5(FAP10) & 25038.9 & 25400.0 & 14239.3 & 34552.3 & 20313.0 \\
\hline D1-E2(FAP10) & 25227.0 & 25460.0 & 15059.5 & 33805.8 & 18746.3 \\
\hline D7(FAP10) & 24937.5 & 25484.0 & 15272.0 & 34159.0 & 18887.0 \\
\hline D1-E2(FAP20) & 24910.5 & 25680.0 & 15702.3 & 34071.0 & 18368.7 \\
\hline D1-E1(FAP10) & 25351.9 & 25757.0 & 15478.0 & 34891.3 & 19413.3 \\
\hline D5(FAP30) & 27309.8 & 26807.5 & 18386.2 & 35480.0 & 17093.8 \\
\hline D3(FAP10) & 27219.9 & 27489.0 & 14687.3 & 36650.3 & 21963.0 \\
\hline D3(FAP30) & 27830.6 & 27790.5 & 16247.9 & 37300.8 & 21052.9 \\
\hline D3(FAP40) & 29816.9 & 27934.0 & 17262.8 & 40711.5 & 23448.7 \\
\hline D3(FAP20) & 27223.9 & 28307.5 & 14602.5 & 36395.1 & 21792.6 \\
\hline D4(FAP40) & 29780.8 & 29129.0 & 19642.0 & 37569.0 & 17927.0 \\
\hline D5(FAP40) & 29569.6 & 29700.0 & 20479.8 & 36915.8 & 16436.0 \\
\hline
\end{tabular}


Table 32 - Steel's Comparison of Ten-Print Matcher Throughput by Device and Probe FAP Size, vs Control (D1-E1).

\begin{tabular}{|c|c|c|c|c|}
\hline Contrast & $\begin{array}{c}\text { Hodges- } \\
\text { Lehmann } \\
\text { location shift }\end{array}$ & Simultaneous $95 \% \mathrm{Cl}$ & 0 & $\mathrm{p}$-value \\
\hline D1-E2(FAP30) - D1-E1(FAP40) & 824.0 & -3511.0 to 5158.0 & II & 1.0000 \\
\hline D1-E2(FAP40) - D1-E1(FAP40) & 658.0 & -3652.0 to 4962.0 & in & 1.0000 \\
\hline D1-E1(FAP30) - D1-E1(FAP40) & -52.0 & -4376.0 to 4267.0 & 10 & 1.0000 \\
\hline D1-E2(FAP20) - D1-E1(FAP40) & -3824.0 & -8019.0 to 349.0 & - & 0.0978 \\
\hline D1-E1(FAP20) - D1-E1(FAP40) & -3989.0 & -8161.0 to 146.0 & $\overline{\mathbf{0}}$ & 0.0669 \\
\hline D2(FAP40) - D1-E1(FAP40) & -7215.5 & -11583.0 to -2877.0 & 口 & $<0.0001$ \\
\hline D2(FAP30) - D1-E1(FAP40) & -7427.0 & -11811.0 to -3105.0 & $\mathbf{\square}$ & $<0.0001$ \\
\hline D2(FAP20) - D1-E1(FAP40) & -11483.0 & -15699.0 to -7333.0 & 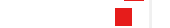 & $<0.0001$ \\
\hline D1-E1(FAP10) - D1-E1(FAP40) & -15761.0 & -19658.0 to -11909.0 & $\square$ & $<0.0001$ \\
\hline D1-E2(FAP10) - D1-E1(FAP40) & -15937.0 & -19869.0 to -12066.0 & $\overline{\mathbf{z}}$ & $<0.0001$ \\
\hline D2(FAP10) - D1-E1(FAP40) & -22861.0 & -26886.0 to -18927.0 & $\square$ & $<0.0001$ \\
\hline D3(FAP40) - D1-E1(FAP40) & -31075.0 & -34468.0 to -27717.0 & - & $<0.0001$ \\
\hline D3(FAP30) - D1-E1(FAP40) & -31317.0 & -34695.0 to -27975.0 & $\overline{-}$ & $<0.0001$ \\
\hline D3(FAP20) - D1-E1(FAP40) & -35313.0 & -38616.0 to -32054.0 & D & $<0.0001$ \\
\hline D3(FAP10) - D1-E1(FAP40) & -43182.0 & -46393.0 to -40073.0 & च & $<0.0001$ \\
\hline D4(FAP30) - D1-E1(FAP40) & -44036.0 & -47283.0 to -40825.0 & $\overline{\mathbf{a}}$ & $<0.0001$ \\
\hline D4(FAP40) - D1-E1(FAP40) & -44408.0 & -47659.0 to -41209.0 & $\bar{\square}$ & $<0.0001$ \\
\hline D4(FAP20) - D1-E1(FAP40) & -47006.0 & -50227.0 to -43863.0 & 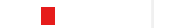 & $<0.0001$ \\
\hline D8(FAP40) - D1-E1(FAP40) & -49495.0 & -52648.0 to -46410.0 & [ & $<0.0001$ \\
\hline D8(FAP30) - D1-E1(FAP40) & -49671.0 & -52839.0 to -46588.0 & [ & $<0.0001$ \\
\hline D8(FAP20) - D1-E1(FAP40) & -51151.0 & -54325.0 to -48081.0 & $\overline{\mathbf{a}}$ & $<0.0001$ \\
\hline D4(FAP10) - D1-E1(FAP40) & -52199.0 & -55382.0 to -49129.0 & 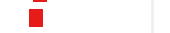 & $<0.0001$ \\
\hline D6(FAP30) - D1-E1(FAP40) & -52776.0 & -56050.0 to -49649.0 & च & $<0.0001$ \\
\hline D6(FAP40) - D1-E1(FAP40) & -52895.0 & -56153.0 to -49763.0 & घ & $<0.0001$ \\
\hline D6(FAP20) - D1-E1(FAP40) & -53359.0 & -56625.0 to -50201.0 & $\overline{\mathbf{a}}$ & $<0.0001$ \\
\hline D5(FAP30) - D1-E1(FAP40) & -55274.0 & -58494.0 to -52201.0 & च & $<0.0001$ \\
\hline D5(FAP40) - D1-E1(FAP40) & -55331.0 & -58561.0 to -52264.0 & $\bar{\square}$ & $<0.0001$ \\
\hline D8(FAP10) - D1-E1(FAP40) & -55349.0 & -58527.0 to -52287.0 & च & $<0.0001$ \\
\hline D6(FAP10) - D1-E1(FAP40) & -56445.0 & -59756.0 to -53270.0 & 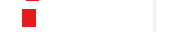 & $<0.0001$ \\
\hline D5(FAP20) - D1-E1(FAP40) & -56453.0 & -59663.0 to -53384.0 & $\bar{\square}$ & $<0.0001$ \\
\hline D5(FAP10) - D1-E1(FAP40) & -59375.0 & -62584.0 to -56282.0 & - & $<0.0001$ \\
\hline D7(FAP40) - D1-E1(FAP40) & -62344.0 & -65755.0 to -59072.0 & & $<0.0001$ \\
\hline D7(FAP30) - D1-E1(FAP40) & -62873.0 & -66312.0 to -59562.0 & & $<0.0001$ \\
\hline D7(FAP20) - D1-E1(FAP40) & -64739.0 & -68219.0 to -61377.0 & & $<0.0001$ \\
\hline D7(FAP10) - D1-E1(FAP40) & -67235.0 & -70778.0 to -63796.0 & & $<0.0001$ \\
\hline
\end{tabular}

Table 33 - Steel's Comparison of Mobile ID Matcher Throughput by Device and Probe FAP Size, vs Control (D1-E1).

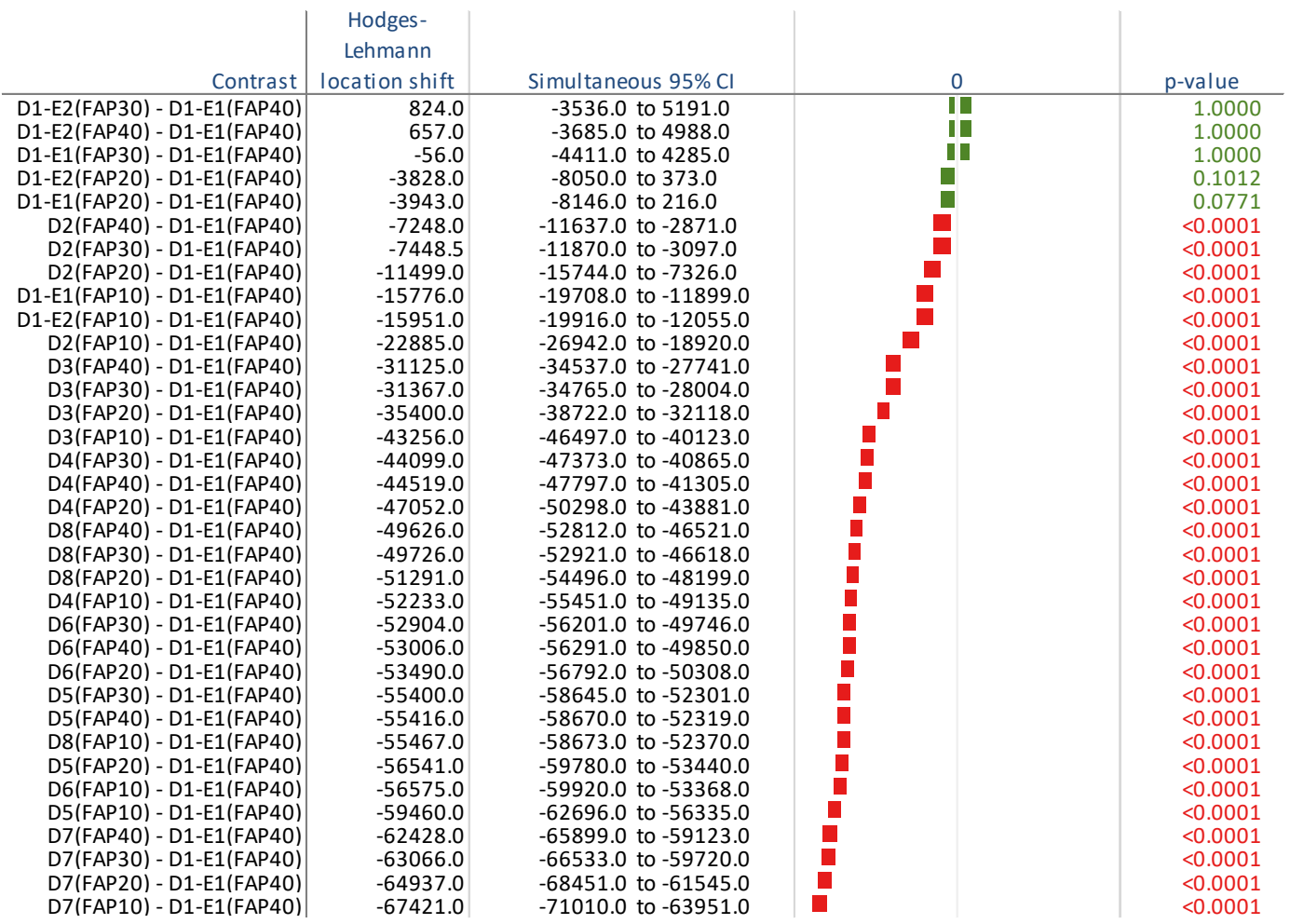




\section{Conclusions}

Based on observations in [7],[8] a number of contactless capture devices were found to have captured superfluous data, artifacts and distortion around the periphery of the captured fingerprint images, as well as not being able to consistently capture certain fingers from each hand presented to them.

We hypothesized that reducing the number of contactless captured fingers submitted (from the full set available down to 6, 4 or even as few as 2 ) by systematically eliminating some of the fingers that the contactless devices traditionally had difficulty capturing may help eliminate a potential source of error, and yield an improvement in the matcher's performance with that transaction. It was hypothesized that reduction in finger count may also improve the service time from the matcher.

Next, we hypothesized that cropping contactless fingerprint images slightly may eliminate superfluous data and distortion in the edges of the contactless captured print thereby eliminating another source of error and improving the ability to match the biometric transaction. It was hypothesized that reduction in size may also improve the matcher service time.

This study set out to examine the above hypotheses by examining the operational impact of utilizing contactless fingerprint imagery with reduced spatial area or reduced finger count on a fingerprint matcher's accuracy as well as throughput.

Examining the impact of reduced finger counts at operational thresholds (See 4.9) shows that there was a general trend of worsening FNIR as the number of probe fingers was reduced on both matchers (Table 11 and Table 12). This trend however was not universal in that while most cases performed worse, 36 of the cases showed an improvement (drop in FNIR) for the contactless cases.

For a deeper dive on the impact of reduced finger counts on FNIR, we next examined both the TenPrint and Mobile ID matchers at the same arbitrary thresholds (See 4.9) to eliminate any bias as a result of operational threshold tuning. The trend worsening FNIR as the number of probe fingers was reduced was observed on both the Ten-Print (Table 13) and Mobile ID (Table 14) matchers. Similar to observations at operational thresholds, worsening FNIR trends with reduced image sizes at arbitrary thresholds was not universal and 40 of the cases showed an improvement (drop in FNIR).

Findings from examinations of finger count reduction failed to show a clear pattern for the cases that improved via reduction of finger counts. The cases that improved however present an opportunity for device-specific guidance (for example, in the case of $D 2 \triangleleft D 5$, the findings show $24 \%$ FNIR reduction by reducing the probe finger count for device D5 from 8 to 2 fingers at the low confidence threshold on the Mobile ID matcher, see Table 14). Also, a differential examination of all cases (Table 15) showed that the Mobile ID matcher had an operational advantage with respect to measured FNIR on 31 cases, while the Ten-Print matcher had an operational advantage on 18 cases. This operational advantage may make the Mobile ID optimized matcher a better choice for systems that operate on both Ten-Print and Mobile ID caseloads.

Examining the impact of reduced fingerprint size on performance of the Ten-Print and Mobile ID matchers (Table 20) showed a worsening FNIR trend as the image size was reduced. This trend was not universal, and there were notable trends in particular devices improving in FNIR performance by image size reduction. One notable example is that of matching D6 to the control biometric reference database (D1-E1). Reducing D6 images sizes in cases of FAP40-to-FAP30 showed an improvement in all 4 of the cases examined (Table 20). Furthermore, at the low confidence threshold $\mathrm{T}=5000$, the TenPrint Matcher showed a net-improvement across the cases being reduced from FAP40-to-FAP30 ( 9 cases improved and 7 degraded, see Table 21). This again presents opportunities for operational tuning of system for device specific cases to improve FNIR.

Next, examination of matcher throughput with respect to reduced finger counts (from 8 fingers to 6, 4 or 2 fingers), showed a linear decrease in the median matcher service time with reduced finger counts on both the Ten-Print matcher (Table 26, Figure 12a) and the Mobile ID matcher (Table 27, Figure 12 b). 
Examination of cases with reduced image size (from FAP40 to FAP30, FAP20 and FAP10), did not show a clear trend. The case that had the lowest service time for the Ten-Print matcher was that of FAP30 (Table 28) with a median service time of $35714 \mathrm{~ms}$. For the Mobile ID matcher, the case with the lowest service time was that of FAP40 (Table 29) with a median service time of 35503ms.

Examination of matcher service time distribution with reduced image sizes (Figure $12 \mathrm{c}$ and d) shows that while the populations were nearly the same, the difference between the service time trials was nonetheless statistically significant for all trials (Table 28, Table 29) at $(\mathrm{p}<0.05)$. Operational optimization considerations may need to take into account the relatively low return on an investment on strategies to improve throughput through image size reduction. 


\section{Acknowledgements}

We would like to thank Mary F. Theofanos (NIST) and Susanne M. Furman (NIST) for their tireless dedication and perseverance for the data collection that made this study possible. Next, we would like to thank Gregory P. Fiumara (NIST) and John M. Splain (Biometrics Guru LLC) for their time, advice, and candor in reviewing the contents of this study, and providing many meaningful suggestions to help make this a better report.

We would also like to thank the 200 Federal employees who volunteered their time and biometric data to make this study possible. 


\section{References}

\begin{tabular}{|c|c|}
\hline$[1]$ & $\begin{array}{l}\text { FBI/CJIS, "Electronic Biometric Transmission Specification (EBTS) Technical and Operational Update (TOU) } 10.0 .9 \\
\text { Redlines", Federal Bureau of Information Criminal Justice Information Systems, Clarksburg, WV, May 22, 2018, } \\
\text { https://www.fbibiospecs.cjis.gov/Document/Get?fileName=EBTS\%20TOU\%2010 } 0 \text { 9 Final.pdf. }\end{array}$ \\
\hline$[2]$ & $\begin{array}{l}\text { Special Publication (NIST SP) - 500-290e3: “American National Standard for Information Systems — Data } \\
\text { Format for the Interchange of Fingerprint, Facial \& Other Biometric Information, ANSI/NIST-ITL 1-2011 } \\
\text { NIST Special Publication 500-290 Edition 3. August 22, 2016. } \\
\text { http://nvlpubs.nist.gov/nistpubs/SpecialPublications/NIST.SP.500-290e3.pdf }\end{array}$ \\
\hline [3] & $\begin{array}{l}\text { Chambers, John; William Cleveland, Beat Kleiner, and Paul Tukey (1983). Graphical Methods for Data Analysis. } \\
\text { Wadsworth }\end{array}$ \\
\hline [4] & $\begin{array}{l}\text { Hollander, M., Wolfe, D. A., \& Chicken, E. (2014). Nonparametric statistical methods (Third edition.). John Wiley \& } \\
\text { Sons, Inc., 256-265 }\end{array}$ \\
\hline [5] & $\begin{array}{l}\text { Wood, S.S., Wilson, C.L.: Studies of Plain-to-Rolled Fingerprint Matching Using the NIST Algorithmic Test Bed (ATB). } \\
\text { From the National Institute of Standards \& Technology (NISTIR 7112) (April 2004), } \\
\text { https://doi.org/10.6028/NIST.IR.7112 }\end{array}$ \\
\hline [6] & $\begin{array}{l}\text { S.L. Cheng, G. Fiumara, C. Watson, PFTII report. Plain and rolled fingerprint matching with proprietary templates. } \\
\text { NISTIR } 7821 \text { (2011), https://doi.org/10.6028/NIST.IR.7821. Retrieved 07/17/2020. }\end{array}$ \\
\hline [7] & $\begin{array}{l}\text { Libert, J., Grantham, J., Bandini, B., Ko, K., Orandi, S., Watson, C., "Interoperability Assessment 2019: Contactless-to- } \\
\text { Contact Fingerprint Capture", NISTIR 8307, National Institute of Standards and Technology, Gaithersburg, MD. } \\
\text { https://doi.org/10.6028/NIST.IR.8307 Retrieved 6/20/2020. }\end{array}$ \\
\hline [8] & $\begin{array}{l}\text { Orandi, S., Libert, J., Bandini, Ko, K., J., Grantham, B., Watson, C., "Evaluating the Operational Impact of Contactless } \\
\text { Fingerprint Imagery on Matcher Performance", NISTIR 8315, National Institute of Standards and Technology, } \\
\text { Gaithersburg, MD. https://doi.org/10.6028/NIST.IR.8315 Retrieved 6/27/2021. }\end{array}$ \\
\hline [9] & $\begin{array}{l}\text { Tabassi, E., Watson, C., Fiumara, G., Salamon, W., Flanagan, P., and Cheng, S. L., "Performance evaluation of fingerprint } \\
\text { open-set identification algorithms," in Proc. Int'I Joint Conf. Biometrics, 2014, pp. 1-8 Retrieved 07/15/2020. }\end{array}$ \\
\hline [10] & $\begin{array}{l}\text { Fiumara G, Flanagan P, Grantham J, Ko, K, Marshall K, Schwarz M, Tabassi E, Woodgate B, Boehnen C (2018) National } \\
\text { Institute of Standards and Technology Special Database 302: Nail to Nail Fingerprint Challenge. NIST Technical Note } \\
\text { 2007. https://doi.org/10.6028/NIST.TN.2007. Retrieved 07/17/2020. }\end{array}$ \\
\hline [11] & $\begin{array}{l}\text { Robert G. D. Steel. (1959). A Multiple Comparison Rank Sum Test: Treatments versus Control. Biometrics, 15(4), 560-572. } \\
\text { doi:10.2307/2527654 }\end{array}$ \\
\hline$[12]$ & 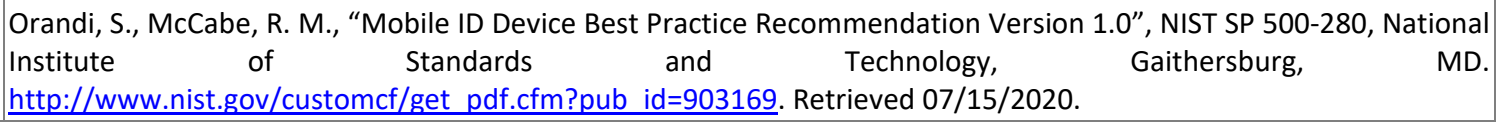 \\
\hline
\end{tabular}

